DRAFT VERSION NOVEMBER 3, 2018

Preprint typeset using LTEX style emulateapj v. 12/16/11

\title{
AN X-RAY AND MULTIWAVELENGTH SURVEY OF HIGHLY RADIO-LOUD QUASARS AT Z > 4: JET-LINKED EMISSION IN THE BRIGHTEST RADIO BEACONS OF THE EARLY UNIVERSE
}

\author{
Jianfeng Wu ${ }^{1,2,3}$, W. N. BrandT ${ }^{1,2}$, Brendan P. Miller ${ }^{4}$, Gordon P. GARmire ${ }^{1}$, Donald P. Schneider ${ }^{1,2}$, \\ CRISTIAN VIGNALI ${ }^{5,6}$ \\ ApJ in press
}

\begin{abstract}
We present a systematic study of the X-ray and multiwavelength properties of a sample of 17 highly radioloud quasars (HRLQs) at $z>4$ with sensitive X-ray coverage from new Chandra and archival Chandra, XMM-Newton, and Swift observations. Eight of the new and archival observations are reported in this work for the first time. New Chandra observations of two moderately radio-loud and highly optically luminous quasars at $z \gtrsim 4$ are also reported. Our HRLQ sample represents the top $\sim 5 \%$ of radio-loud quasars in terms of radio loudness. We found that our HRLQs have an X-ray emission enhancement over HRLQs at lower redshifts (by a typical factor of $\approx 3$ ), and this effect, after controlling for several factors which may introduce biases, has been solidly estimated to be significant at the 3-4 $\sigma$ level. HRLQs at $z=3-4$ are also found to have a similar X-ray emission enhancement over $z<3$ HRLQs, which supports further the robustness of our results. We discuss models for the X-ray enhancement's origin including a fractional contribution from inverse Compton scattering of cosmic microwave background photons. No strong correlations are found between the relative X-ray brightness and optical/UV emission-line rest-frame equivalent widths (REWs) for radio-loud quasars. However, the line REWs are positively correlated with radio loudness, which suggests that relativistic jets make a negligible contribution to the optical/UV continua of these HRLQs (contrary to the case where the emission lines are diluted by the relativistically boosted continuum). Our HRLQs are generally consistent with the known anti-correlation between radio loudness and X-ray power-law photon index. We also found that the two moderately radio-loud quasars appear to have the hardest X-ray spectra among our objects, suggesting that intrinsic X-ray absorption $\left(N_{\mathrm{H}} \sim 10^{23} \mathrm{~cm}^{-2}\right)$ may be present. Our $z>4$ HRLQs generally have higher X-ray luminosities than those for the composite broad-band spectral energy distributions (SEDs) of HRLQs at lower redshift, which further illustrates and supports the X-ray emission enhancement of $z>4$ HRLQs. Some of our HRLQs also show an excess of mid-infrared emission which may originate from the synchrotron emission of the relativistic jets. None of our $z>4$ HRLQs is detected by the Fermi LAT two-year survey, which provides constraints on jet-emission models.
\end{abstract}

Subject headings: galaxies: active — galaxies: nuclei — galaxies: high-redshift — jets: galaxies — X-rays: galaxies

\section{INTRODUCTION}

X-ray studies of $z>4$ active galactic nuclei (AGNs) over the past $\approx 12 \mathrm{yr}$ have greatly improved understanding of the growth of the first supermassive black holes (SMBHs) in the early Universe, thanks to unprecedentedly powerful X-ray observatories as well as wide-field surveys in the optical/UV and radio bands. Chandra and XMM-Newton, along with other X-ray missions, have observed more than 100 quasars at $z>4$, the majority of which were first identified by widefield optical/UV surveys, e.g., the Sloan Digital Sky Survey (SDSS; York et al. 2000), or radio surveys, e.g., the Faint Images of the Radio Sky at Twenty-Centimeters (FIRST; Becker et al. 1995) survey and the NRAO VLA Sky Survey (NVSS;

\footnotetext{
jfwu@astro.psu.edu

${ }^{1}$ Department of Astronomy \& Astrophysics, The Pennsylvania State University, 525 Davey Lab, University Park, PA 16802, USA

${ }^{2}$ Institute for Gravitation and the Cosmos, The Pennsylvania State University, University Park, PA 16802, USA

${ }^{3}$ Harvard-Smithsonian Center for Astrophysics, 60 Garden Street, Cambridge, MA 02138, USA

${ }^{4}$ Department of Astronomy, University of Michigan, Ann Arbor, MI 48109, USA

${ }^{5}$ Dipartimento di Astronomia, Università degli Studi di Bologna, Via Ranzani 1, I-40127 Bologna, Italy

${ }^{6}$ INAF - Osservatorio Astronomico di Bologna, Via Ranzani 1, I40127 Bologna, Italy
}

Condon et al. 1998). The quasar population is divided into radio-quiet and radio-loud subcategories based on the apparently bi-modal distribution of the radio-loudness parameter (e.g., Ivezić et al. 2004)! Which was defined by Kellermann et al. (1989) as $R=f_{5 \mathrm{GHz}} / f_{4400} \AA$ (rest-frame; see $\S 3$ for a detailed definition). Radio-quiet quasars (RQQs) have $R<10$, while radio-loud quasars (RLQs) are those with $R \geqslant 10$.

The basic X-ray properties of $z>4 \mathrm{RQQs}$ are now well established (e.g., Kaspi et al. 2000; Brandt et al. 2001, 2002; Vignali et al. 2001, 2003a, 2003b, 2005; Shemmer et al. 2005, 2006). The $z>4$ RQQs have remarkably similar X-ray properties to those of RQQs at lower redshift, which is notable given the strong evolution of the quasar population over cosmological timescales (e.g., Richards et al. 2006b; Croom et al. 2009; Jiang et al. 2009). These results suggest the mode of SMBH growth via accretion does not strongly evolve with redshift.

RLQs feature powerful jets that originate near the SMBH

\footnotetext{
7 There is an ongoing debate regarding the bi-modality of the radioloudness distribution of quasars (e.g., Kellermann et al. 1989; Ivezić et al. 2002, 2004; Cirasuolo et al. 2003; Singal et al 2011, 2012). Some recent studies with new approaches, e.g., investigating the loci of quasars in the optical/UV spectroscopic parameter space (Zamfir et al. 2008) or the quasar radio luminosity function (Kimball et al. 2011b) found two distinct populations with different radio properties, supporting the dichotomy of radio-loud vs. radio-quiet quasars (but also see, e.g., Broderick \& Fender 2011).
} 
and may extend up to hundreds of kpc. These jets possess bulk relativistic velocities on at least sub-kpc scales and generate strong radio emission that is particularly pronounced in lowinclination (highly beamed, foreshortened, core-dominated) RLQs. RLQs usually show an enhancement of nuclear X-ray emission compared to RQQs, likely due to a contribution from the spatially unresolved jet (e.g., Worrall et al. 1987; Miller et al. 2011; and references therein). Previous X-ray studies of representative RLQs at $z \gtrsim 4(R \approx 40-400$; e.g., Bassett et al. 2004; Lopez et al. 2006; Saez et al. 2011) have shown that the factor of X-ray emission enhancement of these objects is similar to that of low-redshift RLQs of similar radio loudness. These results limit any redshift dependence of the nuclear X-ray emission in RLQs (consistent with earlier studies to moderate redshifts; e.g., Worrall et al. 1987). The lack of strong redshift evolution argues against a dominant X-ray generation mechanism for the nuclear jet-linked contribution whereby relativistic jet electrons upscatter cosmic microwave background photons into the X-ray band (the IC/CMB model; e.g., Tavecchio et al. 2000; Celotti et al. 2001). While photon fields associated with the quasar are expected to be more relevant on small scales $(\approx 0.1-2 \mathrm{kpc})$, for $z=4$ coredominated RLQs (for which $1^{\prime \prime}$ is 7.1 projected kpc and typical inclinations of $5-7^{\circ}$ correspond to deprojected lengths $\sim 10$ times greater) the spatially unresolved X-ray emission potentially includes contributions from the jet to tens of $\mathrm{kpc}$. The IC/CMB model is often proposed to explain the X-ray knot emission from low-redshift large-scale RLQ jets studied with Chandra, but here too the consequent predicted strong increase with redshift in the X-ray luminosities of jet knots (absolute or relative to jet radio or non-IC/CMB core X-ray emission; e.g., Schwartz 2002) is to date not observed (e.g., Bassett et al. 2004; Lopez et al. 2006; Marshall et al. 2011; Saez et al. 2011). While some degree of IC/CMB emission is required, the relative contribution (and associated spatial scales) to the observed X-ray emission is currently unclear.

Some $z>4$ quasars with the highest values of radio loudness $(R \gtrsim 400)$ have been studied individually in X-rays (e.g., PMN J0525-3343, Worsley et al. 2004a; Q 0906+6930, Romani 2006; RX J1028.6-0844, Yuan et al. 2005; GB 1428+4217, Worsley et al. 2004b; GB 1508+5714, Siemiginowska et al. 2003, Yuan et al. 2003, 2006). These objects are often referred to as "blazars" due to their extreme radio loudnesses and other properties $\$$ Most of the previous studies were focused on their X-ray spectral properties. These objects often show soft X-ray spectral flattening; the fraction of objects with such flattening appears to rise with redshift (e.g., Fiore et al. 1998; Page et al. 2005; Yuan et al. 2006). This flattening has been proposed to be caused by intrinsic X-ray absorption with a column density of $N_{H} \sim(1-3) \times 10^{22} \mathrm{~cm}^{-2}$ (but also see Behar et al. 2011). This level of intrinsic X-ray absorption is also found in one $z>4$ quasar with moderate radio loud-

\footnotetext{
8 The term "blazar", including subcategories of BL Lac objects and optically violently variable quasars (e.g., see $\$ 1.3$ of Krolik 1999), has been defined based on a set of criteria, including the equivalent widths of emission lines, the non-thermal jet-linked contribution to the optical continuum, broadband variability, the radio spectral shape, and the polarization. However, this terminology has sometimes been applied either inconsistently or based on incomplete information. For our objects, it is often not feasible to classify them clearly as "blazars" based on available data. All of them are broad-line quasars (i.e., not BL Lac objects). All but one $(J 0913+5919)$ of our objects have flat radio spectra (see 3 . In this work, we have made minimal use of the term "blazar". Instead we define a highly radio-loud quasar sample based on radio loudness (see 2 for details).
}

ness (SDSS J0011+1446; Saez et al. 2011). An alternative mechanism for the soft X-ray spectral flattening is the bulk Comptonization of broad-line photons by relativistic plasma moving along the RLQ jet (e.g., Begelman \& Sikora 1987; Sikora et al. 1997). Celotti et al. (2007) studied the timedependent spectra generated by this mechanism and reproduced the soft X-ray flattening feature in the XMM-Newton spectrum of GB 1428+4217. Volonteri et al. (2011) studied hard-X-ray-selected high-redshift blazars and found a deficit of RLQs at $z>3$ compared to the expectations from the number of blazars. Possible explanations of this deficit require either a decrease of average bulk Lorentz factor in relativistic jets or an increase by a factor of 2-10 in the number of very massive black holes $\left(M_{\mathrm{BH}}>10^{9} M_{\odot}\right)$ at high redshifts (derived from the radio luminosity function). They stressed the importance of finding high-redshift blazars which could provide constraints on the SMBH-dark halo connection and structure formation.

Although these highly radio-loud quasars (HRLQs hereafter) are rare, they produce among the most powerful relativistic jets in the Universe. These remarkable objects have required the larger sky coverage of modern wide-field opti$\mathrm{cal} / \mathrm{UV}$ and radio surveys to be discovered in sufficient numbers to assemble a statistically meaningful sample at high redshift. Current major X-ray missions provide coverage of the 2-40 keV energy band in the rest frame of $z>4$ HRLQs. Studies of the X-ray and broad-band properties of high-redshift HRLQs provide probes of the jet-launching and radiation mechanisms in the early stages of cosmic evolution (e.g., Fabian et al. 1998, 1999). However, there has not been a systematic survey to investigate the general properties of these remarkable HRLQs at $z>4$. Miller et al. (2011; M11 hereafter) studied the X-ray properties of a large, diverse sample of RLQS 9 with wide ranges of redshift and radio loudness. However, HRLQs at $z>4$ only made up $\lesssim 1 \%$ of their full sample. In this work, we conduct the first X-ray survey of a sample of HRLQs at $z>4$ using new X-ray observations by Chandra and sensitive archival X-ray data. Our science goals include the following: (1) assess any X-ray emission enhancement of these objects and compare them to HRLQs at lower redshift; (2) provide constraints on the basic X-ray spectral properties of the newly observed Chandra targets, and study the relation between X-ray spectral properties and radio loudness; (3) investigate relations between relative X-ray brightness, radio loudness, and optical/UV emission-line properties; and (4) study the broad-band spectral energy distributions (SEDs) of our objects.

In $\$ 2$ we describe the selection of our sample of HRLQs at $z>4$. In $\$ 3$ we detail the X-ray observations and the data analyses. Overall results and discussion are presented in $\$ 4$ Throughout this paper, we adopt a cosmology with $H_{0}=70.5 \mathrm{~km} \mathrm{~s}^{-1} \mathrm{Mpc}^{-1}, \Omega_{\mathrm{M}}=0.274$, and $\Omega_{\Lambda}=0.726$ (e.g., Komatsu et al. 2009).

\section{SAMPLE SELECTION}

We began our sample selection from the SDSS Data Release 7 (DR7; Abazajian et al. 2009) quasar catalog (Schneider et al. 2010) which covers $9380 \mathrm{deg}^{2}$ of sky area. We searched for $z \gtrsim 4$ RLQs satisfying one or both of the following criteria: (1) $\log R>2.5(R \gtrsim 320)$; (2) $M_{i}<-29$.

\footnotetext{
${ }^{9}$ M11 defined "RLQs" as quasars with $R \geqslant 100$, while objects with $10 \leqslant$ $R<100$ were classified as radio-intermediate quasars (RIQs). To simplify the terminology, we refer to all quasars with $R \geqslant 10$ as RLQs in this work.
} 
Seven objects were first selected via this method. We also added another object $(\mathrm{J} 0741+2520)$ which does not have SDSS spectroscopy, but was confirmed as a very luminous $\left(M_{i}=-29.04\right) z=5.194$ RLQ in follow-up optical/UV spectroscopy of radio-selected SDSS sources (McGreer et al. 2009). Among the SDSS-covered $z \gtrsim 4$ RLQs without sensitive X-ray coverage, these eight objects have the most remarkable radio and/or optical properties. They are ideal targets, with high radio loudness, high optical luminosity, and high redshift, for economical Chandra snapshot observations. Six of the eight objects were awarded Chandra time in Cycle 12 (see Table 1 for an X-ray observation $\log$ ). Four of the six targets $(\mathrm{J} 1026+2542, \mathrm{~J} 1412+0624, \mathrm{~J} 1420+1205$, and $\mathrm{J} 1659+2101$ ) have radio loudness $\log R>2.5$. We define this radio-loudness value as the threshold for being "highly radio loud". RLQs satisfying this criterion represent the top $\sim 5 \%$ of the RLQ population in terms of radio loudness (see the dash-dotted curve in Fig. (1). We note that Sbarrato et al. (2012b) have also recently studied $\mathrm{J} 1026+2542$, claiming that it is a blazar. The other two targets $(\mathrm{J} 0741+2520$ and $\mathrm{J} 1639+4340$ ) are only moderately radio loud (with $\log R=$ 1.06 and $\log R=1.62$, respectively), but they are the most optically luminous $\left(M_{i}<-29\right)$ RLQs at $z \gtrsim 4$ that have sensitive X-ray coverage (see Fig. 1).

In addition to our Chandra Cycle 12 targets, we further searched for other HRLQs at $z>4$ satisfying our $\log R$ criterion in the sky area north of $\delta=-40^{\circ}$ using the NASA/IPAC Extragalactic Database (NED) TH The sky area north of $\delta=$ $-40^{\circ}$ is covered by the $1.4 \mathrm{GHz}$ NVSS survey, and thus we are able to calculate the radio loudness for each object. In fact, for typical $z>4$ RLQs identified in current wide-field surveys $\left(m_{i} \lesssim 21\right)$, if an object satisfies our $\log R>2.5$ criterion for being a HRLQ, it should have been detected by NVSS according to the NVSS sensitivity $(\sim 2.5 \mathrm{mJy})$. Therefore, our selection method should not introduce incompleteness owing to the radio-flux limit 1 For objects with both FIRST and NVSS detections, we adopt the radio-flux values from the FIRST catalog due to its better sensitivity; the radio flux values in the FIRST and NVSS catalogs are generally consistent with each other. A total of 24 HRLQs at $z>4$ were identified. We checked for sensitive archival X-ray coverage for these $z>4$ HRLQs by Chandra, XMM-Newton, ROSAT T2 or Swift. Thirteen have sensitive archival X-ray coverage (see Table 11 13 while the other 11 do not (see Table 2) 14 Table 1 and Table2 together provide a complete list of known HRLQs at $z>4$ in the sky area north of $\delta=-40^{\circ}$. All of the 13 objects having sensitive archival X-ray coverage are detected

\footnotetext{
${ }^{10}$ http://ned.ipac.caltech.edu/.

${ }^{11}$ However, it is still possible that some relevant HRLQs at $z>4$ have not been identified due to the lack of optical/UV spectroscopic follow up. Therefore it is not possible to generate a fully complete sample of $z>4$ HRLQs at present with available databases.

12 We required the ROSAT observations to be pointed observations (i.e., not ROSAT All-Sky Survey) with an exposure time greater than $5 \mathrm{ks}$ and an off-axis angle less than $19^{\prime}$ (i.e., within the inner ring of the PSPC detector) to achieve adequate sensitivity. However, none of the $z>4$ HRLQs is covered by ROSAT observations satisfying these criteria.

13 Only one known $z>4$ HRLQ (PKS B1251-407) with sensitive X-ray coverage lies south of $\delta=-40^{\circ}$, and thus is not included in our sample. See Yuan et al. (2006) for the X-ray properties of this object.

${ }^{14}$ The NED lists SDSS J112429.62 +283125.8 as a $z=4.38$ quasar based on SDSS DR6 data. However, the redshift of this object provided in the SDSS DR7 quasar catalog is $z=1.36$. Therefore we do not consider this object as a $z>4$ HRLQ although it satisfies our $\log R$ criterion. This object does not have sensitive X-ray coverage.
}

in X-rays; all of them were targeted in their relevant X-ray observations except for SDSS J1235-0003. The archival Chandra, XMM-Newton, or Swift observations of four objects (PMN J1155-3107, SDSS J1235-0003, GB 1713+2148, and PMN J1951+0134) are reported in this work for the first time. Some of the archival objects have been classed as "blazars" and were covered by multiple X-ray observations (see \$1). They were usually selected from radio (e.g., Green-Bank $6 \mathrm{~cm}$, GB6, Gregory et al. 1996; the Parkes-MITNRAO survey, PMN, Griffith \& Wright 1993) or X-ray (e.g., the ROSAT All Sky Survey, Voges et al. 1999) surveys. The individual X-ray properties of these blazars have been studied in detail (see the last column of Table 1 for references). In this work, we combine the 13 archival sources and the 4 newly observed HRLQs to form a sample to perform general systematic studies of HRLQs at high redshift. Our sample of HRLQs at $z>4$ with sensitive X-ray coverage thus includes 17 objects, eight of which have their X-ray properties presented for the first time in this work.

We searched for high-resolution radio images of these objects obtained by the Very Long Baseline Array (VLBA) or Very Long Baseline Interferometry (VLBI); seven of them have available high-resolution images. Two objects have point-like source profiles and no indications of extended radio emission (J0913+5919, Momjian et al. 2004; J1235-0003, Momjian et al. 2004). Another three objects have clear extended radio emission on milliarcsecond (mas) scales from relativistic jets although their extended radio emission only contributes $<10 \%$ of the total radio flux (Q 0906+6930, Romani et al. 2004; GB 1508+5714, Cheung 2004, Helmboldt et al. 2007; GB 1428+4217, Cheung et al. 2012). The extended radio emission of GB $1508+5714$ coincides with the extended X-ray emission, showing the signature of a relativistic jet (Cheung 2004). The extended radio feature of GB $1428+4217$ in its VLA imaging at observed-frame 1.4 GHz and $4.9 \mathrm{GHz}$ (Cheung et al. 2012) lies 3.6" away from the core. It also coincides with the extended X-ray emission found in its Chandra observation. GB $1428+4217$ furthermore shows mas-scale extended radio emission in its VLBI images at observed-frame $2.3 \mathrm{GHz}$ and $8.6 \mathrm{GHz}$. The extended radio emission of $\mathrm{J} 1026+2542$ is on $\mathrm{a} \approx 20$ mas scale; it contributes $\approx 40 \%$ of the total radio flux (Helmboldt et al. 2007). The mas-scale extended radio emission of the other object, $\mathrm{J} 1659+2101$, contributes $\approx 30 \%$ of the total radio flux at observed-frame $1.6 \mathrm{GHz}$, while the image of this object at observed-frame $5 \mathrm{GHz}$ shows no evidence of extended radio emission (Frey et al. 2010) 15 The five objects with extended radio emission have higher radio loudness on average $(\langle\log R\rangle=3.21 \pm 0.23)$ than the three objects without extended radio emission $(\langle\log R\rangle=2.81 \pm 0.25)$.

Besides the 17 HRLQs at $z>4$, we will also separately discuss the two highly optically luminous RLQs observed by Chandra in Cycle 12 even though they do not satisfy the "highly radio loud" criterion. All of our 19 objects are among the most-luminous RLQs in both the radio and optical/UV bands (see Fig. 2). The monochromatic luminosity ranges of

15 We also found high-resolution radio images for two $z>4$ HRLQs without sensitive X-ray coverage listed in Table 2] The VLBI image of $\mathrm{J} 0813+3508$ shows jet-like resolved structure at observed-frame $1.6 \mathrm{GHz}$, which contributes $\approx 1 / 4$ of the total flux, while its image at observed-frame $5 \mathrm{GHz}$ does not have evidence of extended emission (Frey et al. 2010). $\mathrm{J} 1242+5422$ has extended radio emission in both of its VLBI images at observed-frame $1.6 \mathrm{GHz}$ and $5 \mathrm{GHz}$, where the extended emission only contributes $\approx 3 \%$ of the total fluxes (Frey et al. 2010). 
our objects are $10^{33}-10^{36} \mathrm{erg} \mathrm{s}^{-1} \mathrm{~Hz}^{-1}$ in the radio (at restframe $5 \mathrm{GHz}$ ) and $10^{30}-10^{33} \mathrm{erg} \mathrm{s}^{-1} \mathrm{~Hz}^{-1}$ in the optical/UV (at rest-frame $2500 \AA$ ). GB $1713+2148$ has remarkable properties even compared to other HRLQs in our sample. It is much fainter (by a factor of $\approx 6$ ) in the optical band, and it has the highest radio loudness (see Fig. 11. Its radio loudness is also higher than those of most of the objects (99\%) in the full sample of M11. All of our objects are spectroscopically confirmed broad-line quasars by either the SDSS or other observations. They have precise redshift measurements based on their broad emission lines (e.g., Ly $\alpha$ and/or C IV). The available SDSS spectra of nine objects (five Chandra Cycle 12 targets and four archival sources) are shown in Fig. 3.

\section{X-RAY DATA ANALYSIS}

The six RLQs at $z>4$ targeted by Chandra in Cycle 12 were observed with the S3 CCD of the Advanced CCD Imaging Spectrometer (ACIS; Garmire et al. 2003). The Chandra data were reduced using standard CIAO v4.3 routines. We generated $\mathrm{X}$-ray images for the observed-frame soft (0.5-2.0 keV), hard (2.0-8.0 keV), and full (0.5-8.0 keV) bands using ASCA grade 0, 2, 3, 4, and 6 events. The source detection was performed using the WAVDETECT algorithm (Freeman et al. 2002) with a detection threshold of $10^{-6}$ and wavelet scales of $1, \sqrt{2}, 2,2 \sqrt{2}$, and 4 pixels. All targets were clearly detected by Chandra within $0.6^{\prime \prime}$ of the optical coordinates. To assess possible extended X-ray emission from a putative jet, we followed the method in $\S 2.3$ of Bassett et al. (2004) requiring a minimum of $\approx 3$ nearby counts offset from the core by $\approx 2^{\prime \prime}$ along roughly the same direction. None of our targets shows evidence for extended X-ray emission. The X-ray counts were measured with aperture photometry using the IDL APER procedure. The aperture radius was adopted as $1.5^{\prime \prime}$ for each source $(\approx 95 \%$ enclosed energy for the soft band, $\approx 90 \%$ enclosed energy for the hard band; aperture corrections were applied). The background counts were retrieved from an annular region with inner and outer radii of twice and three times the aperture radius, and were scaled to the source-aperture area. All background regions are free of X-ray sources. Table 3 lists the X-ray counts in the three bands defined above, as well as the band ratio (defined as the ratio between hard-band and soft-band counts) and effective power-law photon index for each source. The effective photon index was derived from the band ratio with the Chandra PIMMS 16 tool, under the assumption of a power-law model with only Galactic absorption.

The X-ray properties of the archival objects were obtained from the literature (see Table 1) except for those objects listed in Table 3 for which their archival Chandra, XMM-Newton, or Swift observations have not been previously published. For objects with multiple X-ray observations, we chose the Chandra/XMM-Newton observation with the longest exposure time $\square$ This approach should not introduce any biases in the following considerations regarding the X-ray emission strength. Vignali et al. (2003a) reported a tentative detection of GB $1713+2148$ using a ROSAT HRI observation. This object was later observed with Chandra ACIS in Cycle 5 (PI: L. Gurvits). We processed the Chandra data for GB 1713+2148 using the same method as described in the previous paragraph.

\footnotetext{
16 http://cxc.harvard.edu/toolkit/pimms.jsp

17 We did not use the XMM-Newton observation with the longest exposure time for GB $1428+4217$ because this object was undergoing an exceptional radio flare (see Worsley et al. 2006).
}

The X-ray flux of GB $1713+2148$ in the Chandra epoch is consistent with that in the ROSAT epoch, showing that this object did not have any strong X-ray variability.

SDSS J1235-0003 was serendipitously covered by an XMM-Newton observation on 2010 July 01 . We performed data reduction and processing with standard XMM-Newton Science Analysis System (SAS; v10.0.0) routines. X-ray images were generated for the observed-frame soft, hard, and full bands for the MOS1 and MOS2 detectors; this object is not covered by the pn detector. Source detection was carried out using the EBOXDETECT procedure. This object was detected only in the MOS1 full-band image at a $\approx 3 \sigma$ level, and thus we only used the MOS1 image for its X-ray photometry (it was not detected in the MOS2 images because it is very close to the CCD edge). We filtered the events file by removing the periods of background flaring (49\% of the total exposure time). The aperture radius for the photometry $\left(53.0^{\prime \prime}\right)$ was the $90 \%$ enclosed-energy radius at $1.5 \mathrm{keV}$ based on the point spread function (PSF) of the MOS1 detector at an off-axis angle of $12.8^{\prime}$. The upper limits on X-ray counts in the soft and hard bands were calculated as $3 \sqrt{N}$, where $N$ is the total counts in the aperture. The vignetting was corrected by calculating the effective exposure time $(4.2 \mathrm{ks})$ at the source location based on the exposure map.

Another two archival objects, PMN J1155-3107 and PMN J1951+0134, were targeted by the Swift X-ray Telescope (XRT; Burrows et al. 2005) in two (ObsIDs: 00036263001/2, total exposure time $5.1 \mathrm{ks}$ ) and three (ObsIDs: $00036791001 / 2 / 3$, total exposure time $10.4 \mathrm{ks}$ ) observations, respectively. For each object, we first generated the exposure maps for all XRT events files using the XRTEXPOMAP routine. We then merged the event files and exposure maps for each object using the XSELECT and XIMAGE packages, respectively. Similar to the approach for the Chan$d r a$ and XMM-Newton data, XRT images were created for the observed-frame soft, hard, and full bands. Source detection was performed with the WAVDETECT algorithm with a detection threshold of $10^{-6}$. PMN J1155-3107 is detected in the full band and soft band, while PMN J1951+0134 is detected in all three bands. Aperture photometry was performed using aperture radii of 59.6" and 64.9", which are the $90 \%$ enclosed-energy radii in the full band based on the PSF of the XRT detector at off-axis angles of $1.0^{\prime}$ and $2.6^{\prime} 18$ respectively. The X-ray photometry of these two archival objects is also listed in Table 3

Table 4 lists the key X-ray, optical, and radio properties of our sample:

Column (1): The name of the quasar.

Column (2): The apparent SDSS $i$-band magnitude of the quasar. For objects covered by the SDSS footprint, the values are obtained either from the SDSS DR7 quasar catalog (BEST photometry; Schneider et al. 2010) or from the SDSS database. For other objects, the $i$-band magnitude was converted from $\mathrm{AB}_{1450(1+z)}$ under the assumption of a power-law spectral index of $\alpha_{v}=-0.5\left(f_{v} \propto v^{\alpha_{v}}\right.$; e.g., Vanden Berk et al. 2001). The values of $\mathrm{AB}_{1450(1+z)}$ were calculated from $R$-band magnitudes (from the NED) using the empirical relation of $A B_{1450(1+z)}=R-0.684 z+3.10$ (Kaspi et al. 2000; Vignali et al. 2003a).

\footnotetext{
${ }^{18}$ The Swift mission has excellent rapid pointing capability, while its pointing accuracy is not exceptional. Therefore, it is normal to have off-axis angles of $\approx 1^{\prime}-2^{\prime}$ for targeted observations.
} 
Column (3): The absolute SDSS $i$-band magnitude for the quasar, $M_{i}$, from the SDSS DR7 quasar catalog, which was calculated from the apparent SDSS $i$-band magnitude in Column 2 by correcting for Galactic extinction and assuming a power-law spectral index of $\alpha_{v}=-0.5$.

Column (4): The Galactic neutral hydrogen column density calculated with the Chandra COLDEN"19 tool (Dickey \& Lockman 1990; Stark et al. 1992), in units of $10^{20} \mathrm{~cm}^{-2}$.

Column (5): The count rate in the observed-frame soft X-ray band $(0.5-2.0 \mathrm{keV})$ for the Chandra observed objects, in units of $10^{-3} \mathrm{~s}^{-1}$.

Column (6): The X-ray flux in the observed-frame soft band $(0.5-2.0 \mathrm{keV})$ corrected for Galactic absorption. This measurement was obtained with the Chandra PIMMS tool and is in units of $10^{-14} \mathrm{erg} \mathrm{cm}^{-2} \mathrm{~s}^{-1}$. An absorbed power-law model was used with the photon index $\left(\Gamma_{\mathrm{X}}\right)$ listed in Column 9 and the Galactic neutral hydrogen column density $\left(N_{H}\right)$ listed in Column 4. For objects without $\Gamma_{X}$ information or only with lower limits upon $\Gamma_{\mathrm{X}}$, we adopt $\Gamma_{\mathrm{X}}=1.6$ which is typical for radio-loud quasars (e.g., Page et al. 2005).

Column (7): The X-ray flux density at rest-frame $2 \mathrm{keV}$ generally obtained from the count rate in the observedframe $0.5-2.0 \mathrm{keV}$ band with PIMMS, in units of $10^{-32}$ erg $\mathrm{cm}^{-2} \mathrm{~s}^{-1} \mathrm{~Hz}^{-1}$ and corrected for Galactic absorption. Although for our objects rest-frame $2 \mathrm{keV}$ corresponds to observed-frame $0.3-0.4 \mathrm{keV}$, we chose the standard approach to using the observed-frame $0.5-2.0 \mathrm{keV}$ band because we are able to minimize effects from potential intrinsic X-ray absorption and X-ray instrumental contamination below observedframe $0.5 \mathrm{keV}$, and we can also obtain better photon statistics with the larger numbers of X-ray counts in this band.

Column (8): The logarithm of the quasar luminosity ( $\mathrm{erg} \mathrm{s}^{-1}$ ) in the rest-frame $2-10 \mathrm{keV}$ band corrected for Galactic absorption.

Column (9): The power-law photon index $\left(\Gamma_{\mathrm{X}}\right)$ of the Xray spectrum. For objects listed in Table 3 , the values were obtained from band-ratio analysis (see the last column of Table 3). For the other objects, the values were retrieved from the literature. Some objects have shown X-ray spectral variability (e.g., GB 1428+4217; see Fig. 4 of Worsley et al. 2006). The choice of $\Gamma_{X}$ values do not significantly affect the X-ray flux calculation. We have chosen the Chandra/XMM-Newton observations with the longest exposure time which should best constrain the power-law photon index.

Column (10): The continuum flux density at rest-frame $2500 \AA$ in units of $10^{-27} \mathrm{erg} \mathrm{cm}^{-2} \mathrm{~s}^{-1} \mathrm{~Hz}^{-1}$. For objects appearing in the SDSS DR7 quasar catalog, the values were obtained from the catalog of Shen et al. (2011). For other SDSS covered objects, the flux density was calculated using the SDSS photometry with the composite spectrum for SDSS quasars in Vanden Berk et al. (2001). For objects not covered by the SDSS, the flux density was calculated using the optical photometry from the literature under the assumption of a power-law spectral index of $\alpha_{v}=-0.5$.

Column (11): The logarithm of the monochromatic luminosity $\left(\mathrm{erg} \mathrm{s}^{-1} \mathrm{~Hz}^{-1}\right.$ ) at rest-frame $2500 \AA$, which was calculated from the flux density at rest-frame $2500 \AA$ given in Column 9. A standard cosmological bandpass correction was applied when converting flux into monochromatic luminosity. Column (12): The radio power-law slope $\alpha_{\mathrm{r}}\left(f_{v} \propto v^{\alpha_{\mathrm{r}}}\right)$ be-

\footnotetext{
${ }^{19} \mathrm{http} / / / \mathrm{cxc}$. harvard.edu/toolkit/colden.jsp
}

tween observed-frame $1.4 \mathrm{GHz}$ and $5 \mathrm{GHz}$. The $1.4 \mathrm{GHz}$ flux density was obtained from the FIRST or NVSS surveys, while the $5 \mathrm{GHz}$ flux density was obtained from the GB6 survey, the PMN survey, or from the literature for individual objects (J1639+4340, Holt et al. 2004; Q 0906+6930, Romani 2006; J0913+5919, Momjian et al. 2004). The observations at $1.4 \mathrm{GHz}$ and at $5 \mathrm{GHz}$ are not simultaneous. All objects

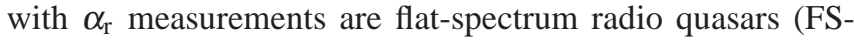
RQs; $\left.\alpha_{\mathrm{r}}>-0.5\right)$ except for J0913+5919. For the five objects without $5 \mathrm{GHz}$ flux measurements, we assume $\alpha_{\mathrm{r}}=0$ to keep consistency with other objects in our sample.

Column (13): The logarithm of the monochromatic luminosity $\left(\mathrm{erg} \mathrm{s}^{-1} \mathrm{~Hz}^{-1}\right.$ ) at rest-frame $5 \mathrm{GHz}$, obtained from the flux density at rest-frame $5 \mathrm{GHz}, f_{5} \mathrm{GHz}$. This quantity was calculated using a radio power-law slope given in Column 12 and the flux density at observed-frame $1.4 \mathrm{GHz}, f_{1.4 \mathrm{GHz}}$, which were obtained from the FIRST or the NVSS surveys. A standard cosmological bandpass correction was also applied.

Column (14): The logarithm of the radio-loudness parameter, given by

$$
\log R=\log \left(\frac{f_{5 \mathrm{GHz}}}{f_{4400 \AA}}\right) .
$$

The denominator, $f_{4400 \AA}$, was calculated from $f_{2500 \AA}$ using an optical power-law slope of $\alpha_{v}=-0.5$. The numerator, $f_{5 \mathrm{GHz}}$, was calculated using the same method as in Column 13. All measures of flux density are per unit frequency. The definition of radio loudness in M11 used $f_{2500 \AA}$ as the denominator. We converted their values to our definition for the scientific analyses described in the following section.

Column (15): The $\alpha_{\text {ox }}$ parameter, defined by

$$
\alpha_{\mathrm{ox}}=\frac{\log \left(f_{2 \mathrm{keV}} / f_{2500 \AA}\right)}{\log \left(v_{2 \mathrm{keV}} / v_{2500 \AA}\right)}=0.384 \log \left(\frac{f_{2 \mathrm{keV}}}{f_{2500 \AA}}\right),
$$

which represents the slope of an assumed power law connecting rest-frame $2500 \AA$ and $2 \mathrm{keV}$. Our UV and X-ray measurements were not simultaneous, and thus the $\alpha_{\mathrm{ox}}$ parameter could be affected by variability.

Column (16): $\Delta \alpha_{\mathrm{ox}, \mathrm{RQQ}}$, defined as

$$
\Delta \alpha_{\mathrm{ox}, \mathrm{RQQ}}=\alpha_{\mathrm{ox}}(\text { measured })-\alpha_{\mathrm{ox}, \mathrm{RQQ}}(\text { expected }) .
$$

The expected $\alpha_{\mathrm{ox}, \mathrm{RQQ}}$ value for a typical RQQ is calculated from the $\alpha_{\mathrm{ox}}-L_{2500} \AA$ correlation given as Equation (3) of Just et al. (2007). The statistical significance of this difference compared to RQQs (given in parentheses) is in units of $\sigma$, which is given in Table 5 of Steffen et al. (2006) as the RMS of $\alpha_{\mathrm{ox}}$ for several ranges of luminosity. The $\Delta \alpha_{\mathrm{ox}, \mathrm{RQQ}}$ parameter quantifies the excess of X-ray emission from the relativistic jet of the RLQs compared to that of RQQs for which the X-ray emission is mainly from the accretion disk and its corona. In 4.2 we will validate the use of $\Delta \alpha_{\mathrm{ox}, \mathrm{RQQ}}$ as a useful diagnostic quantity for HRLQs.

Column (17): $\Delta \alpha_{\text {ox,RLQ}}$, defined as

$$
\Delta \alpha_{\mathrm{ox}, \mathrm{RLQ}}=\alpha_{\mathrm{ox}}(\text { measured })-\alpha_{\mathrm{ox}, \mathrm{RLQ}}(\text { expected }) .
$$

The expected $\alpha_{\mathrm{Ox}, \mathrm{RLQ}}$ value for a typical RLQ is obtained from the $L_{2 \mathrm{keV}}-L_{2500} \AA^{-} L_{5 \mathrm{GHz}}$ correlation based on the full sample in M11 given in their Table 7, which is equivalent to

$$
\alpha_{\mathrm{ox}, \mathrm{RLQ}}=-0.199 L_{2500 \AA}+0.105 L_{5 \mathrm{GHz}}+1.194 .
$$

The $\Delta \alpha_{\text {ox,RLQ }}$ parameter assesses the relative X-ray brightness of a RLQ compared to typical RLQs (mostly at $z=0.3-2.5$ and with a median redshift of $z=1.4$ ) with similar optical/UV 
and radio luminosities that have both disk/corona-linked and jet-linked X-ray emission.

\section{RESULTS AND DISCUSSION}

\subsection{Enhanced X-ray Emission at High Redshift}

4.1.1. The Relative X-ray Brightness of $z>4 H R L Q s$

To assess the enhancement of the X-ray emission of our objects at $z>4$ compared to similar objects at lower redshift, we compare their distributions of $\alpha_{\mathrm{ox}}, \Delta \alpha_{\mathrm{ox}, \mathrm{RQQ}}$, and $\Delta \alpha_{\text {ox,RLQ }}$ to those of RLQs at $z<4$. Fig. 4 shows the positions of our objects and the typical RLQs in M11 in the $\log R-\left(\alpha_{\mathrm{ox}}, \Delta \alpha_{\mathrm{ox}, \mathrm{RQQ}}, \Delta \alpha_{\mathrm{ox} . \mathrm{RLQ}}\right)$ planes. Our objects generally occupy the same region as the typical RLQs in the $\log R-\alpha_{\text {ox }}$ plane (see the top panel of Fig. (4). However, our sample appears to have an excess of objects with stronger $\mathrm{X}$-ray emission in the $\log R-\left(\Delta \alpha_{\mathrm{OX}, \mathrm{RQQ}}, \Delta \alpha_{\mathrm{ox}, \mathrm{RLO}}\right)$ planes. The thick black lines in the middle panel of Fig. 4 show the mean $\Delta \alpha_{\text {ox,RQQ }}$ values of M11 objects binned by radio loudness $(\Delta \log R=0.2$ per bin). Fifteen out of our 17 HRLQs at $z>4$ have $\Delta \alpha_{\mathrm{ox} . \mathrm{RQQ}}$ values greater than the mean value of the corresponding $\log R$ bin. Similarly, 14 of our 17 HRLQs

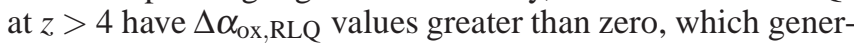
ally represents the mean $\Delta \alpha_{\mathrm{ox}, \mathrm{RLQ}}$ value of M11 objects (see the bottom panel of Fig. (4). We will show later that the generally higher $\Delta \alpha_{\mathrm{OX}, \mathrm{RQQ}}$ and $\Delta \alpha_{\mathrm{OX}, \mathrm{RLQ}}$ values of our objects are caused by stronger X-ray emission rather than weaker opti$\mathrm{cal} / \mathrm{UV}$ emission (see the broad-band SED studies on our objects in 44.4). It is worth noting that the X-ray weakest object in our $z>4$ HRLQ sample (J0913+5919; see the red square with the smallest $\Delta \alpha_{\mathrm{ox}, \mathrm{RQQ}}$ and $\Delta \alpha_{\mathrm{ox}, \mathrm{RLQ}}$ values in Fig. (4) has a steep radio spectral slope $\left(\alpha_{\mathrm{r}}=-0.67\right)$, while the others with available $\alpha_{\mathrm{r}}$ values are all FSRQs.

To verify this excess quantitatively, we obtained the mean values and statistical distributions of $\Delta \alpha_{\mathrm{ox}, \mathrm{RQQ}}$ and $\Delta \alpha_{\mathrm{ox}, \mathrm{RLQ}}$ for our HRLQs and a comparison sample consisting of objects in the full sample 20 of M11 with $\log R>2.5$ and $z<4$. Our sample, which includes objects originally selected in the optical (7 objects; e.g., SDSS), radio (9 objects; e.g., GB6 and PMN), or X-ray (1 object; from the ROSAT All-Sky Survey) bands, is somewhat heterogeneous. To minimize potential selection biases (e.g., the most exceptional objects being preferentially targeted in X-ray observations), we need a sample containing most of the known $z>4$ HRLQs regardless of their selection methods and having nearly complete $X$-ray coverage. To achieve this goal, we chose an opticalmagnitude cut of $m_{i}<20$ on all known $z>4$ HRLQs north of $\delta=-40^{\circ}$ (see Table 1 and Table 2). There are 15 HRLQs at $z>4$ satisfying this $m_{i}$ cut, and 12 of them have sensitive $\mathrm{X}$-ray coverage (and thus are included in our sample). For the three HRLQs without sensitive X-ray coverage, two of them $(\mathrm{J} 0813+3508$ and $\mathrm{J} 1242+5422$, both optically selected) were in our original Chandra Cycle 12 proposal, but were not awarded Chandra time (see $\$ 2$ ). These two objects have the highest redshifts, optical brightnesses, and optical luminosities among those $z>4$ HRLQs without sensitive X-ray coverage (see Table 2). The $i$-band magnitude of the other HRLQ (J2220+0025, optically selected) is close to our $m_{i}$ cut. To maintain consistency, we also applied the $m_{i}<20$ criterion to our comparison sample from M11. There are 283 objects in total in our comparison sample, including $269 \mathrm{X}$-ray detected

\footnotetext{
20 The full sample of M11 contains a mixture of objects that are optically selected $(\sim 80 \%)$, radio selected $(\sim 15 \%)$, or X-ray selected $(\sim 5 \%)$.
}

objects and 14 objects with X-ray upper limits.

The mean values of $\Delta \alpha_{\mathrm{OX}, \mathrm{RQQ}}$ and $\Delta \alpha_{\mathrm{OX}, \mathrm{RLQ}}$ shown in Table 5 were calculated using the Kaplan-Meier estimato 21 implemented in the Astronomy Survival Analysis (ASURV) package (e.g., Lavalley et al. 1992). These mean values indicate that HRLQs at $z>4$ have stronger X-ray emission (by a factor of $\approx 3$ on average) than those at $z<4$ with similar UV and/or radio luminosity. The distributions of $\Delta \alpha_{\mathrm{OX}, \mathrm{RQQ}}$ and $\Delta \alpha_{\text {OX,RLQ }}$ are shown in Fig. 5 The Peto-Prentice test (e.g., Latta 1981), also implemented in the ASURV package, was employed to determine whether the $z>4$ and $z<4$ HRLQs follow the same $\Delta \alpha_{\mathrm{OX}, \mathrm{RQQ}}$ and $\Delta \alpha_{\mathrm{OX}, \mathrm{RLQ}}$ distributions. The Peto-Prentice test is preferred over other two-sample tests because it is the least affected by factors such as unequal sample sizes (e.g., Latta 1981) which do exist in our study. The distribution of $\Delta \alpha_{\mathrm{OX}, \mathrm{RQQ}}$ for our objects is significantly different from that of the HRLQs at $z<4$. The null-hypothesis (i.e., the two samples following the same distribution) probability is only $1.21 \times 10^{-4}$. The two-sample test for the $\Delta \alpha_{\text {ox.RLQ }}$ distribution reaches a similar conclusion; the null-hypothesis probability is slightly higher $\left(1.37 \times 10^{-4}\right)$, but it still shows that the two samples follow different distributions at a $>3 \sigma$ level. One might speculate that the significant differences of the $\Delta \alpha_{\text {ox }}$ distributions are caused by a "tail" of $z>4$ objects with extraordinary X-ray brightness. However, Fig. 5 suggests that the entire $\Delta \alpha_{\mathrm{ox}}$ histograms of our $z>4$ HRLQs are shifted toward high $\Delta \alpha_{\text {ox }}$ values relative to those of $z<4$ HRLQs (also see Fig. 4). Therefore, our sample of HRLQs shows a significant X-ray emission enhancement at $z>4$ over those HRLQs at lower redshift. However, one should keep in mind that although we have compiled a sample of $z>4$ HRLQs with nearly complete X-ray coverage using all available databases, potential selection biases could still exist. For example, although we have assembled all known $z>4$ HRLQs north of $\delta=-40^{\circ}$, this is still not a fully complete sample (see Footnote 11). Therefore, in the next subsection we describe a variety of tests of the robustness of our results.

\subsubsection{Robustness Tests}

In order to test the robustness of our results against selection issues, we carried out further two-sample analyses by constructing other comparison samples or controlling for a variety of relevant parameters (see results in Table 5):

1. Many of our $z>4$ HRLQs are radio selected, while the majority of our comparison sample from M11 is optically selected. To minimize the effects of different selection methods, we first compare our radio-selected $z>4$ HRLQs with a radio-selected subsample in M11. We chose their "Einstein" supplemental sample (see $\S 2.2 .1$ of M11) which was primarily radio selected. The $\mathrm{X}$-ray observations of these objects were first presented in Worrall et al. (1987), and we transform their luminosities to our adopted cosmology. We also incorporate improved X-ray coverage for a handful of objects, as in M11. All of these objects have $z<4$ and $m_{i}<2022$ we further require them to be HRLQs (i.e., $\log R>2.5$ ). Our $z>4$ HRLQs have significantly different $\Delta \alpha_{\text {ox }}$ distributions (both $\Delta \alpha_{\mathrm{OX}, \mathrm{RQQ}}$ and $\Delta \alpha_{\mathrm{OX}, \mathrm{RLQ}}$ ) from those of

${ }^{21}$ The Kaplan-Meier estimator is applicable to censored data; note some of the M11 data points are censored.

${ }^{22}$ The $m_{i}$ values were estimated from their monochromatic luminosities at rest-frame $2500 \AA$. 
this radio-selected comparison sample at $\mathrm{a} \approx 3 \sigma$ level.

2. We generate a quasi-radio-selected comparison sample following $\S 2.1 .1$ of M11 by only selecting those objects that were targeted by SDSS spectroscopy owing to being FIRST radio sources (i.e., with the "FIRST" flag set in the SDSS quasar catalog) Our $z>4$ HRLQs have significantly different $\Delta \alpha_{\text {ox }}$ distributions from those of this "radio-selected" comparison sample at $\mathrm{a} \approx 4 \sigma$ level.

3. As discussed in $\$ 2$, our $z>4$ HRLQs are among the RLQs with the highest radio luminosities; this may introduce a luminosity bias into our sample. As discussed in Ghisellini et al. (2011), for example, more luminous blazars have relatively more hard X-ray emission (because the peak photon energy shifts to lower frequencies), and this could make the observed X-ray flux greater for high-redshift blazars. Therefore, we construct another comparison sample from M11 to have comparable radio luminosity $\left(\log L_{5} \mathrm{GHz}>34\right.$; compare with Fig. 2). After controlling for radio luminosity, the significance of the difference in the $\Delta \alpha_{0 x}$ distributions becomes slightly lower, but still remains at a $\approx 3 \sigma$ level.

4. Similarly, we construct another comparison sample to have comparable optical/UV luminosity $\left(\log L_{2500 \AA}>\right.$ 30.9; compare with Fig. (2) to our $z>4$ HRLQs to avoid optical/UV luminosity bias. The significance of the $\Delta \alpha_{\text {ox }}$ distribution difference is at a $\gtrsim 2.5 \sigma$ level after controlling for optical/UV luminosity.

5. All of our $z>4$ HRLQs with available $\alpha_{\mathrm{r}}$ are FSRQs $\left(\alpha_{\mathrm{r}}>-0.5\right.$; see 33$)$ except for J0913+591124, which may suggest that low-inclination RLQs are overrepresented in our sample. Therefore, for another comparison sample, we selected the M11 full-sample objects with available $\alpha_{\mathrm{r}}$ measurements that satisfy the FSRQ criterion. After controlling for this effect, our results still have $>3.5 \sigma$ significance.

In summary, our finding that $z>4$ HRLQs have a substantial X-ray emission enhancement over HRLQs at lower redshifts remains significant after controlling for those factors which may introduce biases. We also note that, owing to the respectable sizes of the samples being compared, variability effects upon $\Delta \alpha_{\text {ox }}$ distributions should tend to average out (also see $\S 3$ for explanation of how our selection of the X-ray observations utilized was designed to avoid biases due to variability).

It is possible that this X-ray enhancement of HRLQs is not solely confined to $z>4$ but arises at lower redshift. To investigate this briefly, we compared the $\Delta \alpha_{\mathrm{ox}, \mathrm{RQQ}}$ and $\Delta \alpha_{\mathrm{ox}, \mathrm{RLQ}}$ distributions of $z=3-4$ HRLQs ( 9 objects) in the full sample of M11 with those of $z<3$ HRLQs (274 objects), and found they are different at a $\gtrsim 5 \sigma$ level (see Table $6, z=3-4$ HRLQs have both larger $\Delta \alpha_{\mathrm{ox}, \mathrm{RQQ}}$ and $\Delta \alpha_{\mathrm{ox}, \mathrm{RLQ}}$ values which indicate an $\mathrm{X}$-ray emission enhancement by a factor of $\approx 3$ ). However, the $\Delta \alpha_{\mathrm{ox}, \mathrm{RQQ}}$ values of $z=2-3$ HRLQs (39 objects)

\footnotetext{
${ }^{23}$ This is not a true radio-selected sample because, e.g., they were restricted by SDSS magnitude limits for FIRST sources. See §2.1.1 of M11 for details.

${ }^{24}$ This object is not included in our two-sample tests since it does not satisfy $m_{i}<20$.
}

follow a similar distribution to that of $z<2$ HRLQs (235 objects; $0.18 \sigma$ level difference). For the $\Delta \alpha_{\text {ox,RLQ }}$ parameter, although its distribution for $z=2-3$ HRLQs is different from that of $z<2$ HRLQs at a $\approx 2 \sigma$ level, the mean $\Delta \alpha_{\text {ox,RLQ }}$ value of $z=2-3$ HRLQs is slightly lower than that of $z<2$ HRLQs $(-0.030 \pm 0.019$ vs. $0.005 \pm 0.008)$. Therefore, the above tests suggest that the X-ray emission enhancement of HRLQs begins to arise at $z \approx 3$. This statistically independent result from $z=3-4$ also supports the robustness of our findings of a HRLQ X-ray emission enhancement at $z>4$. Furthermore, after combining the samples of $z>4$ HRLQs and $z=3-4$ HRLQs, we find that $z>3$ HRLQs have an X-ray emission enhancement over $z<3$ HRLQs at a $>5.7 \sigma$ significance level corresponding to a null-hypothesis probability of $\approx 10^{-8}$ (also see Table 6).

\subsubsection{Relevant Physical Considerations Regarding the X-ray Emission Enhancement}

Our results suggest that the most-luminous relativistic jets generate stronger X-ray emission in the early universe. Previous studies by Bassett et al. (2004) and Lopez et al. (2006) that included a mix of HRLQs (including a subset of those in our sample) and moderately radio-loud quasars found that highredshift objects did not show substantially stronger X-ray emission compared to a lower-redshift sample of RLQs detected by ROSAT (from Brinkmann et al. 1997). We assess further these previous results by performing a similar two-sample analysis on the moderately radio-loud quasars $(1<\log R<2.5)$ in the full sample of M11 (which includes the moderately radio-loud objects from Bassett et al. 2004 and Lopez et al. 2006). The $\Delta \alpha_{\mathrm{ox}, \mathrm{RQQ}}$ distribution of $z>4$ moderately radio-loud quasars ( 10 objects; all X-ray detected) is different from that of $z<4$ moderately radio-loud quasars (280 objects with $48 \mathrm{X}$-ray upper limits) at a $\approx 2 \sigma$ level, while the $\Delta \alpha_{\mathrm{ox}, \mathrm{RLQ}}$ values of $z>4$ moderately radio-loud quasars follow a similar distribution to that of $z<4$ moderately radioloud quasars $(0.57 \sigma$ level difference). Therefore, there is no firm evidence that moderately radio-loud quasars have a significant X-ray emission enhancement compared to those at lower redshift (recall also from $\$ 1$ that RQQs show no evolution in X-ray properties with redshift). This suggests that the X-ray enhancement in high-redshift HRLQs occurs within the jet-linked component, which likely (e.g., M11) contributes an increasing fraction of the X-ray continuum with increasing radio-loudness values 25

The enhancements in relative nuclear X-ray emission we find for $z>4$ HRLQs are less than would be predicted for an X-ray jet-linked component dominated by IC/CMB emission, which possesses a strong $(1+z)^{4}$ dependence on redshift (e.g., Schwartz 2002; see §1). For example, the median redshift for the RLQs from Miller et al. (2011) with $z<4$, $\log R>2.5$, and $m_{\mathrm{i}}<20$ is $z=1.3$. If the jet-linked contribution to the nuclear X-ray emission is at least $\approx 50 \%$ at $z=1.3$, as is a conservative estimate for RLQs with $\log R>2.5$ (e.g., Zamorani et al. 1981; Figure 7 of M11), then for a pure IC/CMB X-ray jet the overall enhancement in the nuclear $\mathrm{X}$-ray emission at $z=4.4$ would be $\gtrsim 16$, corresponding to an increase in $\Delta \alpha_{\text {ox,RLQ }}$ of 0.46 . However, our $z>4$ HRLQs only show an increase in $\Delta \alpha_{\text {ox,RLQ }}$ of $0.15-0.20$ (see Table 5). Instead, our results are consistent with an X-ray jet-

\footnotetext{
${ }^{25}$ It is obviously also possible, but not required, that the jet-linked X-ray generation mechanism of HRLQs differs from (and is more sensitive to redshift than) that of moderately radio-loud quasars.
} 
linked component in which the fractional contribution from the IC/CMB process at $z=1.3$ is $\lesssim 6 \%$ of the nuclear X-ray emission, with the balance of the jet-linked X-ray emission arising from inverse Compton scattering of other seed photon fields such as radiation from the broad-line region or dust (e.g., Sikora et al. 2009), from the jet synchrotron emission itself (e.g., Sokolov et al. 2004; Meyer et al. 2012 find that synchrotron self-Compton emission is relatively less important in high-powered jets), or from non-cospatial synchrotron radiation in a structured jet (e.g., Migliori et al. 2012). The overall enhancement to the nuclear X-ray continuum then rises more gradually with redshift; at $z=4.4 / 3.0 / 2.0$ RLQs would be X-ray brighter by factors of $\lesssim 2.8 / 1.5 / 1.1$ (relative to a $z=1.3$ template), corresponding to $\Delta \alpha_{\mathrm{Ox}, \mathrm{RLQ}}$ increases of $0.17 / 0.07 / 0.02$.

Another possible explanation for the X-ray enhancement is that an increasing contribution of the X-ray emission from the jets is due to the inverse-Compton scattering of the photon field of the host galaxy; e.g., Hardcastle \& Croston (2011) provided a detailed modeling of upscattering of host-galaxy photon fields into high-energy emission. The X-ray enhancement of our sample can be explained if the host galaxies of high-redshift HRLQs are more luminous in the infrared/optical band than those at lower redshift. This higher infrared/optical luminosity could be generated by the enhanced star-formation activity of high-redshift host galaxies (e.g., Wang et al. 2008, 2011a; Mor et al. 2012). Smail et al. (2012) found that for the high-redshift $(z=3.6-3.8)$ radio galaxies in their study, the luminosity of the extended $\mathrm{X}$-ray halo is correlated with the far-infrared luminosity of the galaxy, which supports the X-ray generation mechanism of inverse Compton scattering of the far-infrared photons produced by star-formation activity. Since the cosmic star formation rate density has a broad plateau starting at $z \sim 1$ and running to $z \sim 4$ (e.g., Hopkins \& Beacom 2006), one might expect in this model the enhancement to emerge at $z \sim 1$ rather than at $z \sim 3-4$. However, the generally massive host galaxies of quasars may show stronger evolution effects at earlier cosmic times owing to cosmic downsizing (e.g. Cowie et al. 1996; Panter et al. 2007). For example, Archibald et al. (2001) found a $(1+z)^{3}$ evolution of the total infrared luminosity of radio galaxies at high redshifts.

The $\alpha_{\mathrm{Ox}}$ and $\Delta \alpha_{\mathrm{Ox}}$ parameters are defined based on the $\mathrm{X}$-ray flux density at rest-frame $2 \mathrm{keV}$, which corresponds to observed-frame $\sim 0.3-0.4 \mathrm{keV}$ for our $z>4$ HRLQs. However, the rest-frame $2 \mathrm{keV}$ flux density was derived from the observed-frame $0.5-2.0 \mathrm{keV}$ count rate (see $\$ 3$, which means that we have extrapolated the X-ray spectrum to restframe $2 \mathrm{keV}$ assuming a single power-law model. It is known that some $z>4$ HRLQs show X-ray spectral flattening below observed-frame $0.5 \mathrm{keV}$, i.e., an X-ray flux deficit compared to a single power-law model (e.g., Yuan et al. 2006). Therefore, the extrapolation process may have over-estimated the $\mathrm{X}$-ray flux density at rest-frame $2 \mathrm{keV}$. Although X-ray spectral flattening has also been found in a few $z<3$ objects (e.g., Page et al. 2005), it would not affect the X-ray flux calculation because rest-frame $2 \mathrm{keV}$ is covered by the observedframe $0.5-2.0 \mathrm{keV}$ band for these objects. To test whether the $\mathrm{X}$-ray emission enhancement we found for $z>4$ HRLQs is caused by this kind of X-ray spectral curvature, we recalculated the $\alpha_{\mathrm{Ox}}$ and $\Delta \alpha_{\mathrm{OX}}$ values for our $z>4$ HRLQs utilized in the two-sample analyses above (i.e., those with $m_{i}<20$ ) from the count rates in the observed-frame ultrasoft band
(0.3-1.0 keV; see results in Table 7) except for the two Swift sources (PMN J1155-3107 and PMN J1951+0134) which do not have adequate counts in the ultrasoft band due to the limited sensitivity of the Swift XRT. These $\alpha_{\text {ox, US values ob- }}$ tained from the ultrasoft band are similar to those we have obtained in $\$ 3$, the mean difference between them is $\left\langle\alpha_{\mathrm{Ox}, \mathrm{US}}-\right.$ $\left.\alpha_{\text {ox }}\right\rangle=-0.03 \pm 0.01$. If we use the $\Delta \alpha_{\text {ox }}$ values calculated from the ultrasoft band ( $\Delta \alpha_{\mathrm{OX}, \mathrm{RQQ}, \mathrm{US}}$ and $\left.\Delta \alpha_{\mathrm{OX}, \mathrm{RLQ}, \mathrm{US}}\right)$ in the two-sample tests above, the $\Delta \alpha_{\text {ox }}$ distributions of $z>4$ HRLQs and $z<4$ HRLQs are still different at a $\gtrsim 3 \sigma$ level. Therefore, X-ray spectral curvature only contributes to a small fraction, if any, of the X-ray emission enhancement of $z>4$ HRLQs over those at lower redshift. In fact, some of the $z>4$ HRLQs without the apparent X-ray spectral curvature (e.g., GB 1508+5714; Yuan et al. 2006) are among the objects we find to have the strongest X-ray enhancements.

Considering the seven $z>4$ HRLQs with high-resolution radio images (see $\$ 2$ ), the five with extended radio emission on mas scales have higher $\Delta \alpha_{\mathrm{ox}, \mathrm{RQQ}}$ values $\left(\left\langle\Delta \alpha_{\text {ox.RQQ }}\right\rangle=0.51 \pm 0.09\right)$ on average than those of the two without extended radio emission $\left(\left\langle\Delta \alpha_{\mathrm{OX}, \mathrm{RQQ}}\right\rangle=0.15 \pm 0.24\right)$. This might be because the objects with extended radio emission have higher average radio loudness (see \$2). However, After considering the strength of their radio emission, the objects with extended radio emission still have a higher average $\Delta \alpha_{\mathrm{Ox}, \mathrm{RLQ}}$ value $\left(\left\langle\Delta \alpha_{\mathrm{ox}, \mathrm{RLQ}}\right\rangle=0.23 \pm 0.09\right)$ than that of the objects without extended radio emission $\left(\left\langle\Delta \alpha_{\mathrm{Ox}, \mathrm{RLQ}}\right\rangle=-0.07 \pm 0.23\right)$ although with substantial uncertainty due to the limited number of objects.

\subsection{Optical/UV Broad Emission Lines}

The optical/UV spectra of quasars at $z>4$ usually show prominent broad emission lines such as $\operatorname{Ly} \alpha+\mathrm{N}$ V and C IV (see Fig. 3). For RLQs, if the optical/UV continuum emission is also beamed following the radio emission, one might expect that the emission-line rest-frame equivalent widths (REWs) would be correlated with the beaming of the radio emission. Therefore, we investigate the relation between emission-line REWs and radio loudness for our sample of HRLQs, and compare this relation to that for the majority of RLQs over a wide range of redshift.

For our $z>4$ HRLQs with SDSS spectroscopy, we obtained measurements of REWs from Diamond-Stanic et al. (2009) and Shen et al. (2011) for $\operatorname{Ly} \alpha+\mathrm{N}$ V and C IV, respectively (see Table 8) 26 Two objects $(\mathrm{J} 1026+2542$ and $\mathrm{J} 1420+1205$ ) with SDSS spectroscopy do not have REW $(\operatorname{Ly} \alpha+\mathrm{N}$ v) measurements in Diamond-Stanic et al. (2009) 2 We measured $\operatorname{REW}(\operatorname{Ly} \alpha+\mathrm{N} v)$ for these two sources following the method in $\S 2$ of Diamond-Stanic et al. (2009). Available REW measurements for other objects without SDSS spectroscopy were also obtained from the literature (see Table 8). Measurements of REW(Ly $\alpha+\mathrm{N} v)$ are usually affected by the presence of the Ly $\alpha$ forest, so our values likely underestimate the intrinsic strength of Ly $\alpha$. Although the number density of Ly $\alpha$ absorbers is known to evolve with redshift (e.g., Weymann et al. 1998; Songaila \& Cowie 2002; Janknecht et al. 2006), this effect will not introduce biases to our correlation analyses of emission-line REWs with radio

26 We also list the values of $\mathrm{J} 1639+4340$ in Table 8 and the following Table 9 for reference despite it is a moderately radio-loud quasar.

27 Diamond-Stanic et al. (2009) measured the REW(Ly $\alpha+\mathrm{N}$ v) values for SDSS DR5 quasars (Schneider et al. 2007) at $z>3$. These two objects are in the SDSS DR7 quasar catalog but not in the DR5 quasar catalog. 
loudness assuming the RLQ radio-loudness distribution does not evolve with redshift. The number of objects in our sample that have emission-line REW measurements is not adequate for reliable correlation analysis. Therefore, we first investigate these correlations for a sample of RLQs across a wide range of redshift, and then see whether our $z>4$ HRLQs follow similar trends. We selected all SDSS DR7 RLQs which have REW(Ly $\alpha+\mathrm{N} \mathrm{v})$ and REW(C IV) measurements from Diamond-Stanic et al. (2009) and Shen et al. (2011), respectively. Fig. 6shows the relation between emission-line REWs and radio loudness, for which highly significant positive correlations are found (see Table 10 for the correlation probabilities). Fig. 6 also shows the best-fit linear correlation in the logarithmic parameter space using the IDL LINFIT procedure,

$\log \operatorname{REW}(\operatorname{Ly} \alpha+\mathrm{N} \mathrm{V})=(1.68 \pm 0.06)+(0.05 \pm 0.02) \log R$,

and

$$
\log \mathrm{REW}(\mathrm{C} \text { IV })=(1.20 \pm 0.02)+(0.16 \pm 0.01) \log R
$$

Our HRLQs generally follow the above positive correlations between emission-line REWs and radio-loudness (see the filled stars and squares in Fig. 6), showing that these correlations do not seem to evolve with redshift. These positive correlations are consistent with the results in Kimball et al. (2011a) on REW(Mg II) and REW(C IV) for SDSS DR5 quasars 28 One possible explanation for them is that the broad emission-line region of RLQs may emit anisotropically (see $\S 6.2$ of Kimball et al. 2011a). Additionally or alternatively, the decrease in synchrotron peak frequency with increasing peak or kinetic luminosity in FSRQs (e.g., Meyer et al. 2011, their Figure 4) might more stringently limit jet dilution of optical emission-line features in more radio-loud quasars. Our results indicate that for HRLQs with broad emission lines (i.e., not BL Lac objects), the beamed relativistic jets generally have negligible contribution to the optical/UV continua (except for a few outliers with exceptionally weak optical/UV emission lines; see below). This is consistent with recent results from SED studies of HRLQs (e.g., Ghisellini et al. 2010) that their optical/UV emission is disk dominated. Furthermore, this finding of a minimal jet-linked contribution to the optical/UV continuum validates the use of $\Delta \alpha_{\text {ox, RQQ }}$ as a diagnostic quantity for our HRLQs; note in Column (16) of Table 4 that the $\alpha_{\mathrm{OX}}-L_{2500} \AA$ correlation for RQQs is utilized.

To assess further the jet contribution to the optical/UV continua of our HRLQs, we calculated the non-thermal dominance parameter (NTD $\left.\equiv L_{\mathrm{obs}} / L_{\mathrm{pred}}\right)$ of the optical/UV continuum following Shaw et al. (2012). $L_{\mathrm{obs}}$ is the observed monochromatic continuum luminosity near an emission line ( $v L_{1350}$ near C IV in our case), while $L_{\text {pred }}$ is the predicted $v L_{1350}$ from the relation between continuum luminosity and emission-line luminosity in Shen et al. (2011; see their Equation 14) obtained from typical quasars. $L_{\text {pred }}$ represents the optical/UV continuum emission from the accretion disk. NTD» 1 means that there is substantial jet-contributed optical/UV continuum. We calculated the NTD values for our

\footnotetext{
${ }^{28}$ Kimball et al. (2011a) found positive correlations between the core radio-to-optical flux ratio $R_{\mathrm{I}}$ and the REW values of Mg II and C IV for SDSS RLQs. The definition of $R_{\mathrm{I}}$ is generally different from that of radio loudness because the latter is defined based on the total radio luminosity. However, the positive correlations in Kimball et al. (2011a) also hold true for their subsample of RLQs without extended radio emission (the "core" morphology class which makes up $\approx 70 \%$ of their full sample; see their Table 8 ). In this case, the definition of $R_{\mathrm{I}}$ is similar to that of radio loudness. Therefore, our results are consistent with those of Kimball et al. (2011a).
}

HRLQs with SDSS spectroscopy covering the C IV line (see Table 9. Three objects (J1420+1205, J1235-0003, and $\mathrm{J} 1325+1123$ ) have NTD values close to unity, supporting our results that the relativistic jets of our HRLQs generally have little contribution to their optical/UV continua. Another object, GB $1508+5714(\mathrm{NTD}=1.3)$, may possibly have some jet-linked optical/UV continuum emission. However, its NTD value is consistent with unity considering the scatter of the relation between $v L_{1350}$ and $L_{\mathrm{C} \text { IV }}(\sim 0.2$ dex; see $\S 3.7$ of Shen et al. 2011). The other two HRLQs (J1412+0624 and $\mathrm{J} 1659+2101$ ) have NTDs $\approx 6$ due to their exceptionally weak $\mathrm{C}$ IV lines that are discussed further below.

For the $\operatorname{REW}(\operatorname{Ly} \alpha+\mathrm{N} v)-\log R$ correlation, it appears that there are a few outliers with exceptionally weak Ly $\alpha+\mathrm{N} \mathrm{v}$ lines (see the upper panel of Fig. 6); they were identified as weak-line quasars (WLQs) by Diamond-Stanic et al. (2009), including one of our Chandra Cycle 12 targets (J1412+0624). Another $z>4$ HRLQ (J1659+2101) in our sample has REW $(\operatorname{Ly} \alpha+\mathrm{N}$ v) values $(17.9 \AA$; see Table 8$)$ close to the WLQ criterion of $\operatorname{REW}(\operatorname{Ly} \alpha+\mathrm{N} \mathrm{v})<15.4 \AA$. These two objects also have weak C IV emission lines. Their REW(C IV) values are below the $3 \sigma$ negative deviation from the mean REW(C IV) of SDSS quasars (Wu et al. 2012). The weak emission lines of these objects may suggest they have a relativistically boosted optical/UV continuum which dilutes the emission lines. However, the broad-band SEDs of these objects (see 44.4) do not show the signature of a relativistically boosted continuum (e.g., a parabolic profile of the SED; Nieppola et al. 2006). It is also possible that the weakness of their emission lines is caused by extreme quasar disk-wind properties as for the radio-quiet weak-line quasars (e.g., Richards et al. 2011; Wu et al. 2011, 2012). One of our moderately radio-loud quasars $(\mathrm{J} 1639+4340)$ also has a weak $\mathrm{C}$ IV line (see Table 8). This object is more likely to fit in the latter scenario; and thus the apparent high NTD value of this object does not indicate that it has substantial jet-contributed optical/UV continuum.

The jet-linked X-ray emission of RLQs is also likely beamed, although probably to a lesser extent compared to jet radio emission (e.g., see $\S 6$ of M11). Therefore, the relation between emission-line REWs and relative X-ray brightness is also worth investigating. A correlation analysis was performed on a sample combining our RLQs and the objects in M11 that have available Ly $\alpha+\mathrm{N}$ V and C IV REW measurements (see Fig.7for the relation between line REWs and relative X-ray brightness of this sample) using Spearman's rankorder analysis in the ASURV package; Spearman's rank-order analysis is usually preferred over Kendall's $\tau$ test for samples where the number of objects is $N \geqslant 30$ The results are shown in Table 10 Only a marginal correlation $(93.3 \%)$ was found between REW(C IV) and $\Delta \alpha_{\text {Ox, RQQ }}$, which is consistent with the result in Richards et al. (2011) that RLQs with weaker $\mathrm{C}$ IV lines also tend to be weaker in X-rays. However, the bottom-left panel ( $\Delta \alpha_{\text {ox.RQQ }}$ vs. C IV REW) of Fig. 7 shows a large scatter in this correlation. No other significant correlations were found between relative $\mathrm{X}$-ray brightness and emission-line strength for the RLQs (see Table 10 and Fig.77).

\subsection{X-ray Spectral Properties}

29 See Appendix A3.3 of the ASURV manual at http://astrostatistics.psu.edu/statcodes/asurv In our work, we choose Spearman's rank-order analysis when sample size $N \geqslant 30$ and Kendall's $\tau$ test when $N<30$. 
Our Chandra Cycle 12 targets do not have sufficient counts for individual X-ray spectral analysis, and thus we obtained their effective power-law photon indices based on their band ratios (see 33 ). The X-ray power-law photon index for RLQs is known to be anti-correlated with $\log R$ (e.g., Wilkes \& Elvis 1987; Reeves \& Turner 2000; Lopez et al. 2006; Saez et al. 2011). Our sample of HRLQs at $z>4$ is generally consistent with this anti-correlation (see Fig. 8). Kendall's $\tau$ test on a sample combining our HRLQs and the moderately radio-loud quasars at $z>4$ in Saez et al. (2011) shows a $2 \sigma$-level anticorrelation between $\Gamma_{\mathrm{X}}$ and $\log R(95.6 \%$ correlation probability). The best-fit correlation for this sample, obtained via the EM linear-regression algorithm in the ASURV package 30 is

$$
\Gamma_{\mathrm{X}}=(1.95 \pm 0.28)+(-0.11 \pm 0.10) \log R \text {. }
$$

This $\Gamma_{\mathrm{X}}-\log R$ relation (the dashed line in Fig. 8) is consistent with that for the $z>2$ RLQs in Saez et al. (2011; see their Equation 1) and with that for the $z<2$ RLQs in Reeves \& Turner (2000; see Equation 2 of Saez et al. 2011), suggesting that this relation does not evolve with redshift. The two moderately radio-loud quasars observed in Chandra Cycle 12 $(\mathrm{J} 0741+2520$ and $\mathrm{J} 1639+4340)$, which do not satisfy our criterion for HRLQs, are outliers from this correlation. Instead of having the softer X-ray spectra generally associated with lower radio loudness, they have the hardest spectra among our objects.

HRLQs often appear to have intrinsic X-ray absorption with $N_{H} \gtrsim 10^{22} \mathrm{~cm}^{-2}$ (e.g., Yuan et al. 2006). Bassett et al. (2004) found evidence of a similar level of intrinsic X-ray absorption for $z>4$ moderately radio-loud quasars. To assess possible indications of intrinsic absorption for our Chandra Cycle 12 targets, we performed joint spectral analyses. We divided our six targets into two groups with $\log R>2.5$ (four objects) and $\log R<2.5$ ( 2 objects), respectively. For each object, the X-ray spectrum in observed-frame $0.5-8.0 \mathrm{keV}$ (corresponding to rest-frame $\approx 2-40 \mathrm{keV}$ ) was extracted from a $3^{\prime \prime}$-radius circular region centered on the X-ray position using the standard CIAO routine PSEXTRACT. Background spectra were extracted from annular regions with inner and outer radii of $6^{\prime \prime}$ and $9^{\prime \prime}$, respectively. All background regions are free of X-ray sources. Joint X-ray spectral fitting was performed with XSPEC v12.6.0 (Arnaud 1996). We applied the $C$-statistic (Cash 1979) in the spectral fitting instead of the standard $\chi^{2}$ statistic because the $C$-statistic is well suited to limited X-ray count scenarios (e.g., Nousek \& Shue 1989). We fit the spectra jointly using two models: (1) a power-law model with a Galactic absorption component represented by the wabs model (Morrison \& McCammon 1983); (2) another model similar to the first, but adding an intrinsic (redshifted to the source rest frame) neutral absorption component, represented by the zwabs model. Each source was assigned its own values of redshift and Galactic neutral hydrogen column density (see Column 4 of Table 4). Table 11 shows the bestfit spectral parameters along with the errors or upper limits at the $90 \%$ confidence level for one parameter of interest $(\Delta C=2.71$; Avni 1976; Cash 1979). The best-fit power-law photon index values are consistent with those from band-ratio analysis (see Table 3). Our joint-fitting procedure is not able to provide tight constraints on the intrinsic X-ray absorption for either highly or moderately radio-loud quasars (see the up-

\footnotetext{
30 This linear-regression algorithm is applicable to censored data. Our sample has two objects with upper limits upon $\Gamma_{\mathrm{X}}$.
}

per limits on intrinsic $N_{H}$ in Table 11 mainly due to the limited number of X-ray counts and their high redshifts. Adding an intrinsic absorption component does not improve the quality of the joint fits.

As discussed above, our two moderately radio-loud quasars appear to have a harder average X-ray spectrum than that of our HRLQs, which appears inconsistent with the known anti-correlation between X-ray power-law photon index and radio loudness. Possible heavy X-ray absorption may exist in these two objects. We estimate the potential intrinsic $\mathrm{X}$-ray absorption for these two moderately radio-loud quasars by assuming their unabsorbed power-law photon indices follow the $\log R-\Gamma_{\mathrm{X}}$ relation (see Equation $8 ; \Gamma_{\mathrm{X} \text {.unabs }}=1.79$ for $\mathrm{J} 0741+2520, \Gamma_{\mathrm{X}, \text { unabs }}=1.74$ for $\mathrm{J} 1639+4340$ ), and then adding an intrinsic X-ray absorption component to make the apparent $\Gamma_{X}$ agree with the values obtained from the bandratio analysis $\left(\Gamma_{X}=1.21\right.$ for $\mathrm{J} 0741+2520, \Gamma_{X}=1.19$ for $\mathrm{J} 1639+4340$; see Table 3). The required intrinsic hydrogen column densities are $N_{\mathrm{H}}=3.3 \times 10^{23} \mathrm{~cm}^{-2}$ for $\mathrm{J} 0741+2520$ and $N_{\mathrm{H}}=2.0 \times 10^{23} \mathrm{~cm}^{-2}$ for $\mathrm{J} 1639+4340$, which are about an order of magnitude higher than the values found for highredshift RLQs in previous studies. The intrinsic X-ray absorption in RLQs is often associated with absorption features in their optical/UV spectra (e.g., Elvis et al. 1998). The optical/UV spectrum of J1639+4340 (see the top-right panel of Fig. 3) shows several C IV and Si IV absorption features, although none of them has sufficient width to be identified as a broad absorption line (BAL). The optical/UV spectrum of J0741+2520 (see Fig. 3 of McGreer et al. 2009) does not show Si IV absorption features; it does not have coverage of the $\mathrm{C}$ IV region.

\subsection{The Radio-to- $\gamma$-ray Spectral Energy Distributions}

To investigate the broad-band SEDs of our RLQs, we gathered photometric data from the following sources:

1. Radio: The $1.4 \mathrm{GHz}$ flux densities are from the FIRST or NVSS surveys; the $5 \mathrm{GHz}$ values are from the GB6 or PMN catalogs or individual observations (see 3 ;) the flux densities at other frequencies were retrieved from the NED.

2. Mid-infrared: From the all-sky catalog of the Wide-field Infrared Survey Explorer (WISE; Wright et al. 2010) 31 All of our objects have WISE detections in at least two bands (W1 at $3.4 \mu \mathrm{m}$ and $\mathrm{W} 2$ at $4.6 \mu \mathrm{m}$ ) except for GB 1428+4217 and PMN J1951+0134. We examined the WISE images of these two objects. There appears to be a source at the optical location of GB $1428+4217$, but it is strongly blended with a nearby brighter source $9^{\prime \prime}$ away. There is no source at the optical location of PMN J1951+0134 in the relevant WISE images. All other objects are free of issues such as source blending, confusion, or mismatching. The high detection fraction $(>17 / 19)$ in the mid-infrared band is remarkable given the high redshifts of our objects.

3. Near-infrared: From the Two Micron All Sky Survey (2MASS; Skrutskie et al. 2006). For the objects having SDSS spectroscopy, we also checked the SDSS DR7 quasar catalog which provides additional deeper

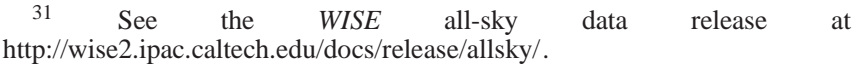


2MASS photometry. Only our two moderately radioloud quasars (J0741+2520 and J1639+4340) have 2MASS detections, likely owing to their exceptional overall luminosities. GB $1428+4217$ has additional near-infrared observations with the United Kingdom Infra-Red Telescope (UKIRT) reported by Fabian et al. (1999).

4. Optical: From the SDSS database and/or the NED. The photometry for the bands that are seriously affected by the Ly $\alpha$ forest is omitted. We do not include any UV photometric information for the same reason.

5. X-ray: From this work.

6. $\gamma$-ray: The upper limits upon $\gamma$-ray luminosities are derived from the two-year survey data of the Large Area Telescope (LAT; Atwood et al. 2009) onboard the Fermi Gamma-Ray Space Telescope. See below for details.

The radio-to- $\gamma$-ray SEDs of our objects are plotted in Fig. 9 ordered by $\log R$ in descending order. We compare the SEDs of our high-redshift HRLQs to those of similar RLQs at lower redshifts. There are two commonly used composite SEDs of RLQs in the literature: Elvis et al. (1994; E94 hereafter) and Shang et al. (2011; S11 hereafter). The E94 SED is biased toward X-ray bright quasars as they required their objects to have X-ray observations with good signal-to-noise ratio (see discussion in $\S 3$ of E94 and $\$ 5.1$ of Richards et al. 2006a). For the S11 SED, although their sample is not X-ray selected, the majority of their RLQs have lower optical/UV luminosities than those of our objects. The optical/UV luminosity can affect the shape of the broad-band SED (see Eqn. 5 for the dependence of $\alpha_{\text {ox }}$ on optical/UV luminosity). The $\alpha_{\text {ox }}$ parameter of RLQs also depends on radio luminosity (see Eqn. 5). Therefore we selected 10 RLQs in the S11 sample with comparable optical/UV luminosity $\left(\log \lambda L_{\lambda}[3000 \AA]>45.9\right)$ and radio-loudness values $(2.9<\log R<3.7)$ to those of our $z>4$ HRLQs. We construct a comparison composite SED using these 10 objects (see the solid lines in Fig. 9). We normalized the comparison SED to the observed data at rest-frame $2500 \AA$ for the following reasons: (1) rest-frame $2500 \AA$ is within a region without strong emission lines; (2) the optical/UV continuum generally does not have a substantial contribution from the jet (except perhaps for a few objects with weak emission lines; see 4.2 ; and (3) when SEDs are normalized at rest-frame $2500 \AA$, the relative positions of X-ray data points directly reflect the $\alpha_{\mathrm{OX}}$ values of the relevant quasars. One caveat about the SEDs is that our multi-band photometry is non-simultaneous, and some HRLQs are known to have strong variability.

The 10 RLQs from S11 used in constructing our comparison SED satisfy our $\log R$ criterion for HRLQs; they are HRLQs at low redshifts $(z<1.4)$. The majority of our HRLQs (11/17) have higher X-ray luminosities than those of the comparison SED. Meanwhile, they do not show weaker optical/UV emission relative to their infrared emission. Therefore, the generally higher $\Delta \alpha_{0 x}$ values of our $z>4$ HRLQs (see 44.1 ) do reflect stronger X-ray emission instead of fainter optical/UV emission. This is an additional illustration of the X-ray emission enhancement of $z>4$ HRLQs compared to HRLQs at lower redshift. The SEDs of RX J1028-0844, GB 1428+4217, and GB 1508+5714 all reach maximal $v L v$ values (over the $10^{9}-10^{19} \mathrm{~Hz}$ range) in the X-ray band. This type of SED has been modeled as the emission generated by Compton scattering of synchrotron emission from the jet itself and/or of a powerful external photon field such as the thermal emission of the accretion process (SSC/EC; see Fabian et al. 1998, 1999; Ghisellini et al. 1998). The SED of Q $0906+6930$ has also been described with a similar model (Romani 2006). Q 0906+6930, along with PMN J0525-3343 and GB 1713+2148, have notably stronger mid-infrared emission than that of the comparison SED, especially in the longer wavelength bands (W3 at $12 \mu \mathrm{m}$ and W4 at $22 \mu \mathrm{m}$ ). This mid-infrared excess may have a contribution from the jet synchrotron radiation (e.g., Ghisellini et al. 1998). An alternative origin for the mid-infrared excess is the thermal emission of circumnuclear dust predicted by the clumpy torus model (e.g., Nenkova et al. 2008). The SEDs of our HRLQs are significantly different from those of typical BL Lac objects which generally have a parabolic profile peaking in the near-infrared band (e.g., Nieppola et al. 2006).

Many blazars are high-energy $\gamma$-ray emitters above $100 \mathrm{MeV}$ (e.g., Hartman et al. 1999). Q 0906+6930 has a tentative claimed detection $(\sim 1.5 \sigma)$ in its $\gamma$-ray observation by the Energetic Gamma-Ray Experiment Telescope (EGRET) onboard the Compton Gamma-Ray Observatory (Romani et al. 2004). The Fermi LAT has an order of magnitude better sensitivity and positional accuracy than EGRET. Romani (2006) suggested Q 0906+6930 should be detected by the Fermi LAT in its first year of operation. However, although the first two-year survey of the Fermi LAT has detected $>800$ blazars at $z \lesssim 3$ (e.g., Ackermann et al. 2011), none of our HRLQs, including Q 0906+6930, is detected at present. We searched the second source catalog of the Fermi LAT (2FGL; Nolan et al. 2012) and found no counterparts for our HRLQs within a $10^{\prime}$ matching radius 32 To obtain upper limits upon $\gamma$-ray luminosity, we first estimated the photon flux limits (in units of photons $\mathrm{cm}^{-2} \mathrm{~s}^{-1}$ ) for all objects based on the point-source flux limit map of the Fermi LAT two-year survey (see Fig. 1 of Ackermann et al. 2011). We converted the photon flux limits to energy flux limits between observedframe $100 \mathrm{MeV}-100 \mathrm{GeV}$ and then flux-density limits at restframe $1 \mathrm{GeV}$, assuming a typical power-law photon index of Fermi-detected FSRQs $\left(\Gamma_{\gamma}=2.4\right.$; see Fig. 18 of Ackermann et al. 2011). The upper limits upon the monochromatic $\gamma$-ray luminosity at rest-frame $1 \mathrm{GeV}$, calculated from the flux-density limits at the same frequency, are in the range of (1.2-2.6) $\times 10^{47} \mathrm{erg} \mathrm{s}^{-1} \mathrm{~Hz}^{-1}$ (see Table 12 and Fig. 9). For some of our $z>4$ HRLQs with strong X-ray enhancements (e.g., Q 0906+6930, RX J1028-0844, GB 1428+4217, and GB $1508+5714$ ), the $\gamma$-ray luminosity limits appear inconsistent with the prediction of the synchrotron-Compton blazar emission model in Romani et al. (2006; see their Fig. 3). Further Fermi LAT survey observations will provide tighter $\gamma$-ray luminosity upper limits (by a factor of $\sim 2$ ) or perhaps detections of $z>4$ blazars, which will help constrain the emission models for blazars at high redshift. Sbarrato et al. (2012a) found a positive correlation between the luminosity of optical/UV broad emission lines and the $\gamma$-ray luminosity for Fermi-detected blazars. This correlation may suggest that the $z>4$ blazars with higher optical/UV emission-line luminosity should be easier to detect with Fermi.

\footnotetext{
${ }^{32}$ We also searched for the $z>4$ HRLQs without sensitive X-ray coverage listed in Table 2 and found none of them having counterparts in the 2FGL catalog.
} 
Six objects with SDSS spectroscopy have available blackhole mass $\left(M_{\mathrm{BH}}\right)$ and Eddington-ratio $\left(L / L_{\mathrm{Edd}}\right)$ estimates (see Table 13 in the catalog of Shen et al. (2011) 33 The bolometric luminosity in Shen et al. (2011) was calculated from the optical/UV luminosity and the bolometric correction from the composite SED of Richards et al. (2006a). Therefore, the bolometric luminosity and the Eddington ratio only account for the emission of the quasar from its accretion activity (i.e., excluding beamed jet emission). All of the six objects have $M_{\mathrm{BH}}>10^{9} M_{\odot}$ except for GB $1508+5714$. These five objects have estimated Eddington ratios between 0.1 and 1, which are typical for SDSS quasars at high redshift (e.g., Shen et al. 2008). GB $1508+5714$ has a smaller $M_{\mathrm{BH}}$ but an Eddington ratio $L / L_{\mathrm{Edd}} \approx 3$. This object is similar to the Fermi-detected FSRQs in Shaw et al. (2012) which have smaller $M_{\mathrm{BH}}$ and higher Eddington ratios than those of optically selected quasars. The objects in Shaw et al. (2012) show significant non-thermal (synchrotron) emission in the optical band associated with the jet. However, GB $1508+5714$ has a smaller NTD value (consistent with unity; see 4.2) than the majority of the objects in Shaw et al. (2012; see their Fig. 3), which suggests that this object does not have substantial jetlinked optical/UV continuum emission. It is worth noting that the $M_{\mathrm{BH}}$ measurements for our objects are all based on the $\mathrm{C}$ IV emission line due to their high redshifts. The $\mathrm{C}$ IV estimator for $M_{\mathrm{BH}}$ is more affected by the quasar disk wind than the $\mathrm{H} \beta$ and $\mathrm{Mg}$ II estimators, and this may lead to errors for individual $M_{\mathrm{BH}}$ measurements (e.g., Shen et al. 2008). Thus, the apparent super-Eddington emission of GB 1508+5714 should not be over-interpreted.

\section{SUMMARY AND FUTURE STUDIES}

We have compiled a sample of 17 highly radio-loud quasars $(\log R>2.5)$ at $z>4$ with sensitive $\mathrm{X}$-ray coverage by Chandra, XMM-Newton, or Swift. Four of them were targeted in new Chandra Cycle 12 observations, while the other 13 objects have sensitive archival X-ray coverage. Our sample of HRLQs represents the top $\sim 5 \%$ of the total RLQ population in terms of radio loudness. They are among the mostluminous quasars in both the radio and optical/UV bands. The X-ray and broad-band properties of our HRLQs are presented and investigated. We have also reported new Chandra Cycle 12 observations of two moderately radio-loud quasars $(1<\log R<2.5)$ at $z \gtrsim 4$ which are among the most optically luminous RLQs. Our main results are summarized as follows.

1. All of our Chandra Cycle 12 targets are detected in X-rays. None of them shows detectable extended X-ray emission. All of the archival objects in our sample of $z>4$ HRLQs were also detected by Chandra, XMM-Newton, or Swift, including four objects for which their archival Chandra (GB 1713+2148), XMM-Newton (SDSS J1235-0003), or Swift (PMN J1155-3107 and PMN J1951+0134) observations are first reported in this work. See $\$ 3$

2. Our HRLQs at $z>4$ show stronger X-ray emission (by a typical factor of $\approx 3$ ) than HRLQs at lower redshift

33 Another HRLQ in our sample, $\mathrm{J} 1026+2542$, has $M_{\mathrm{BH}}$ estimated by Sbarrato et al. (2012b) based on the peak frequency of disk emission and the total disk luminosity. The estimated range is $M_{\mathrm{BH}}=(1.8-4.5) \times 10^{9} M_{\odot}$. Although this object has SDSS spectroscopy, its $M_{\mathrm{BH}}$ cannot be measured based on available emission-line widths because none of the standard lines $(\mathrm{H} \beta, \mathrm{Mg}$ II, and $\mathrm{C}$ IV) is covered due to its high redshift $(z=5.304)$. with similar optical/UV and radio luminosities. This contrasts with the behavior of moderately radio-loud quasars at $z>4$, implying that the high-redshift X-ray enhancement occurs within the jet-linked component that becomes increasingly prominent for HRLQs. We examined possible biases in our analysis and found that our result remains robust after controlling for a variety of potential issues. A similar X-ray emission enhancement is also found for $z=3-4$ HRLQs, which provides statistically independent evidence for our findings. The observed X-ray enhancement is not likely to be caused by the X-ray spectral curvature found in some highredshift HRLQs. See 4.1

3. Our $z>4$ HRLQs are generally consistent with the positive correlations between optical/UV emission-line REWs (Ly $\alpha+\mathrm{N}$ V and C IV) and radio loudness found for the typical RLQ population. These positive correlations suggest that the optical/UV continua of HRLQs usually have a negligible contribution from the relativistic jets (except for perhaps a few outliers with weak emission lines). Our $z>4$ HRLQs do not show strong correlations between the relative X-ray brightness and optical/UV emission-line REWs. See 4.2

4. Our sample of $z>4$ HRLQs generally follows the known anti-correlation between X-ray power-law photon index and radio loudness. However, the two targeted moderately radio-loud quasars, having the hardest X-ray spectra among our objects, appear to be outliers from this correlation, suggesting the possible presence of intrinsic X-ray absorption $\left(N_{\mathrm{H}} \sim 10^{23} \mathrm{~cm}^{-2}\right)$. See 4.3 .

5. The majority of $z>4$ HRLQs have higher X-ray luminosities than those of the matched low-redshift HRLQs used to construct a comparison SED, which further illustrates and supports the X-ray emission enhancement of $z>4$ HRLQs over those at lower redshifts. The SEDs of our objects with the highest X-ray luminosities are consistent with the expectations from SSC/EC models. Some of our HRLQs also show an excess of midinfrared emission which may be contributed by synchrotron radiation from the relativistic jets. None of our HRLQs has been detected by the Fermi LAT in its two-year survey. See 4.4 .

We have performed the first systematic X-ray survey of HRLQs at $z>4$. Our primary new result is the measurement of an increase (by a factor of $\approx 3$ ) in the relative X-ray emission of $z>4$ HRLQs compared to their low-redshift counterparts. This work adds the relative X-ray brightness of HRLQs to the RLQ properties that evolve with redshift, such as the frequency of intrinsic X-ray absorption (see $\$ 1$ ) and perhaps the RLQ fraction (e.g., Jiang et al. 2007; Singal et al. 2012). The number density of hard X-ray selected FSRQs has been found to evolve strongly with redshift, with a peak number density of $z_{\text {peak }} \approx 3-4$ (Ajello et al. 2009). Unfortunately, the sample utilized by Ajello et al. (2009) from the Swift Burst Alert Telescope (BAT; Barthelmy et al. 2005) does not have any FSRQ detections at $z>4$, and thus there is considerable uncertainty in making quantitative comparisons at the high redshifts most applicable to our work. We do note, however, that the SED evolution we find for high-redshift HRLQs (which are primarily FSRQs) will affect the interpretation of 
number-density evolution results for X-ray selected samples. For example, it may help to explain the higher $z_{\text {peak }}$ value found for FSRQs than for general quasars or X-ray selected AGNs.

While the limited increase in X-ray luminosities confirms earlier findings that the small-scale X-ray jet-linked emission is not dominated by IC/CMB emission, our results are consistent with a fractional contribution from IC/CMB $(\sim 6 \%$ of the nuclear X-ray emission at $z=1.3$ ) rising with redshift. This does not conflict with growing evidence that RLQ jets have mildly relativistic bulk velocities beyond several $\mathrm{kpc}$, as inferred from radio core/jet prominences (e.g., $\gamma_{\text {jet }} \approx 1.2-1.5$; Mullin \& Hardcastle 2009), or that various predictions of IC/CMB models are not obviously met in X-ray observations of large-scale RLQ jets (e.g., Hardcastle 2006; Marshall et al. 2011; Massaro et al. 2011; note these studies are based almost exclusively on RLQs with $z<2.5$ ). It may be that IC/CMB emission is relevant only over a limited range of spatial scales (e.g., $\approx 1-5 \mathrm{kpc}$ ), with quasar-related photon fields dominating at smaller distances (e.g., Ghisellini \& Tavecchio 2009 ) and the jet decelerating at larger distances. For example, a drop in the bulk jet velocity from $\beta=0.995$ to $\beta=0.75$, corresponding to a decrease from $\gamma=10$ at $\lesssim 5 \mathrm{kpc}$ to $\gamma=1.5$ at large scales, would act to diminish X-ray IC/CMB emission by a factor of $\approx 44$. Jet deceleration appears to be required in at least some RLQs even in the context of IC/CMB modeling of the large-scale $\mathrm{X}$-ray jet emission, when coupled with VLBI constraints (Hogan et al. 2011). Such deceleration could be partially due to mass entrainment; simulations predict that even in powerful jets the bulk Lorentz factor decreases by a factor of $\sim 2$ on kpc scales (Bowman et al. 1996). Although faster jets are more stable against Kelvin-Helmholtz instabilities (e.g., Perucho et al. 2005), the gradual accumulation of velocity-shear perturbations may similarly act to slow the jet. The Compton-rocket effect (e.g., Ghisellini \& Tavecchio 2010) provides another means of draining jets. Whatever the dominant mechanism, the postulated degree of deceleration could plausibly be accomplished without disrupting or decollimating the jet; for example, in their classic work Kaiser $\&$ Alexander (1997) estimate that a powerful jet with $\gamma_{\text {jet }}=2$ is persistently stable (although see also Wang et al. 2011b). This deceleration possibility can be tested with additional observations of large-scale jets in high-redshift RLQs 34 In a fractional IC/CMB scenario, the enhanced X-ray emission from $z>4$ HRLQs is solely due to the increased energy density of the CMB; the jet bulk velocities, the disk/corona accretion structure, and the host-galaxy properties of individual RLQs are not required to evolve with redshift.
Alternative interpretations of the observed X-ray emission enhancement of $z>4$ HRLQs are also possible. For example, increased host-galaxy star formation could provide additional seed photons for external Compton upscattering in the jet (see $\S 4.1 .3)$. Also, a decrease in the jet bulk Lorentz factor at high redshift (a possibility suggested by Volonteri et al. 2011) might increase the ratio of X-ray to radio emission, particularly in a multi-component jet. It does not appear likely that $\mathrm{X}$-ray spectral curvature drives our results, as the observedframe ultra-soft luminosities are also enhanced. After careful consideration of selection effects, there is no obvious indication that our sample contains a disproportionate number of "extreme" HRLQs, with properties (low inclinations, strong variability) likely to correlate with enhanced X-ray emission.

Future X-ray observations of additional $z>4$ HRLQs, e.g., a Chandra snapshot survey of the 11 objects listed in Table 2, would further improve the sample statistics and better constrain the possible mechanisms for the X-ray enhancement. For example, the HRLQs at $z=3-4$ apparently have a similar level of X-ray enhancement to that of $z>4$ HRLQs, which would not agree with the expectations from the fractional IC/CMB model. However, more objects are needed to reduce the large uncertainties on the mean $\Delta \alpha_{\mathrm{OX}}$ values in current analyses. Identifying potential intrinsic X-ray absorption in our objects, especially for the two moderately radioloud quasars (J0741+2520 and J1639+4340), requires deeper $\mathrm{X}$-ray spectroscopic observations. The ongoing Fermi LAT survey will provide tighter $\gamma$-ray luminosity upper limits or perhaps $\gamma$-ray detections of $z>4$ HRLQs, which will be useful for modeling their SEDs and for constraining the relative strengths of the jet emissions from different mechanisms.

We thank the anonymous referee for constructive comments. We thank M. S. Brotherton, A. C. Fabian, M. J. Hardcastle, R. F. Mushotzky, E. S. Perlman, G. T. Richards, R. W. Romani, and Z. Shang for helpful discussions. We gratefully acknowledge the support of the ACIS Instrument Team contract SV4-74018 (PI: G. P. Garmire) and NASA ADP grant NNX10AC99G (J.W., W.N.B). Funding for the SDSS and SDSS-II has been provided by the Alfred P. Sloan Foundation, the Participating Institutions, the National Science Foundation, the U.S. Department of Energy, the National Aeronautics and Space Administration, the Japanese Monbukagakusho, the Max Planck Society, and the Higher Education Funding Council for England. The SDSS Web site is http://www.sdss.org/

\section{REFERENCES}

Abazajian, K. N., Adelman-McCarthy, J. K., Agüeros, M. A., et al. 2009, ApJS, 182, 543

Ackermann, M., Ajello, M., Allafort, A., et al. 2011, ApJ, 743, 171

Ajello, M., Costamante, L., Sambruna, R. M., et al. 2009, ApJ, 699, 603

Archibald, E. N., Dunlop, J. S., Hughes, D. H., et al. 2001, MNRAS, 323, 417

Arnaud, K. A., 1996, in ASP Conf. Ser. 101, Astronomical Data Analysis

Software and Systems V, ed. G. H. Jacoby \& J. Barnes (San

Francisco:ASP), 17

Atwood, W. B., Abdo, A. A., Ackermann, M., et al. 2009, ApJ, 697, 1071

Avni, Y. 1976, ApJ, 210, 642

\footnotetext{
34 Cheung et al. (2012) interpret the $\mathrm{X}$-ray/radio flux ratios of jets in GB $1428+4217$ and GB $1508+4714$ as supportive of an IC/CMB origin, but require a lower bulk Lorentz factor than is typically inferred from IC/CMB modeling of low-redshift RLQs.
}

Barthelmy, S. D., Barbier, L. M., Cummings, J. R., et al. 2005,

Space Sci. Rev., 120, 143

Bassett, L. C., Brandt, W. N., Schneider, D. P., et al. 2004, AJ, 128, 523

Becker, R. H., White, R. L., \& Helfand, D. J., 1995, ApJ, 450, 559

Begelman, M. C., \& Sikora, M. 1987, ApJ, 322, 650

Behar, E., Dado, S., Dar, A., \& Laor, A. 2011, ApJ, 734, 26

Bowman, M., Leahy, J. P., \& Komissarov, S. S. 1996, MNRAS, 279, 899

Brandt, W. N., Guainazzi, M., Kaspi, S., et al. 2001, AJ, 121, 591

Brandt, W. N., Schneider, D. P., Fan, X., et al. 2002, ApJ, 569, L5

Brinkmann, W., Yuan, W., \& Siebert, J. 1997, A\&A, 319, 413

Broderick, J. W., \& Fender, R. P. 2011, MNRAS, 417, 184

Burrows, D. N., Hill, J. E., Nousek, J. A., et al. 2005, Space Sci. Rev., 120, 165

Cash, W. 1979, ApJ, 228, 939

Celotti, A., Ghisellini, G., \& Chiaberge, M. 2001, MNRAS, 321, L1

Celotti, A., Ghisellini, G., \& Fabian, A. C. 2007, MNRAS, 375, 417

Cheung, C. C. 2004, ApJ, 600, L23

Cheung, C. C., Stawarz, Ł., Siemiginowska, A., et al. 2012, ApJ, 756, L20 
Cirasuolo, M., Celotti, A., Magliocchetti, M., \& Danese, L. 2003, MNRAS, 346,447

Condon, J. J., Cotton, W. D., Greisen, E. W., et al. 1998, AJ, 115, 1693

Cowie, L. L., Songaila, A., Hu, E. M., \& Cohen, J. G. 1996, AJ, 112, 839

Croom, S. M., Richards, G. T., Shanks, T., et al. 2009, MNRAS, 399, 1755

Diamond-Stanic, A. M., Fan, X., Brandt, W. N., et al. 2009, ApJ, 699, 782

Dickey, J. M., \& Lockman, F. J. 1990, ARA\&A, 28, 215

Elvis, M., Wilkes, B. J., McDowell, J. C., et al. 1994, ApJS, 95, 1 (E94)

Elvis, M., Fiore, F., Giommi, P., \& Padovani, P. 1998, ApJ, 492, 91

Fabian, A. C., Iwasawa, K., McMahon, R. G., et al. 1998, MNRAS, 295,

L25

Fabian, A. C., Celotti, A., Pooley, G., et al. 1999, MNRAS, 308, L6

Fabian, A. C., Celotti, A., Iwasawa, K., et al. 2001, MNRAS, 323, 373

Feigelson, E. D., \& Nelson, P. I. 1985, ApJ, 293, 192

Fiore, F., Elvis, M., Giommi, P., \& Padovani, P. 1998, ApJ, 492, 79

Freeman, P. E., Kashyap, V., Rosner, R., Lamb, D. Q., 2002, ApJS, 138, 185

Frey, S., Paragi, Z., Gurvits, L. I., Cseh, D., \& Gabányi, K. É. 2010, A\&A, 524, A83

Garmire, G. P., Bautz, M. W., Ford, P. G., Nousek, J. A., \& Ricker, G. R., Jr. 2003, Proc. SPIE, 4851, 28

Gehrels, N. 1986, ApJ, 303, 336

Ghisellini, G., Celotti, A., Fossati, G., Maraschi, L., \& Comastri, A. 1998,

MNRAS, 301, 451

Ghisellini, G., \& Tavecchio, F. 2009, MNRAS, 397, 985

Ghisellini, G., \& Tavecchio, F. 2010, MNRAS, 409, L79

Ghisellini, G., Tavecchio, F., Foschini, L., et al. 2010, MNRAS, 402, 497

Ghisellini, G., Tagliaferri, G., Foschini, L., et al. 2011, MNRAS, 411, 901

Gregory, P. C., Scott, W. K., Douglas, K., \& Condon, J. J. 1996, ApJS, 103, 427

Griffith, M. R., \& Wright, A. E. 1993, AJ, 105, 1666

Grupe, D., Mathur, S., Wilkes, B., \& Elvis, M. 2004, AJ, 127, 1

Hardcastle, M. J. 2006, MNRAS, 366, 1465

Hardcastle, M. J., \& Croston, J. H. 2011, MNRAS, 415, 133

Hartman, R. C., Bertsch, D. L., Bloom, S. D., et al. 1999, ApJS, 123, 79

Hewett, P. C., \& Wild, V. 2010, MNRAS, 405, 2302

Healey, S. E., Romani, R. W., Cotter, G., et al. 2008, ApJS, 175, 97

Helmboldt, J. F., Taylor, G. B., Tremblay, S., et al. 2007, ApJ, 658, 203

Hogan, B. S., Lister, M. L., Kharb, P., Marshall, H. L., \& Cooper, N. J. 2011, ApJ, 730, 92

Holt, J., Benn, C. R., Vigotti, M., et al. 2004, MNRAS, 348, 857

Hook, I. M., \& McMahon, R. G. 1998, MNRAS, 294, L7

Hook, I. M., McMahon, R. G., Shaver, P. A., \& Snellen, I. A. G. 2002, A\&A, 391, 509

Hopkins, A. M., \& Beacom, J. F. 2006, ApJ, 651, 142

Ivezić, Ž., Menou, K., Knapp, G. R., et al. 2002, AJ, 124, 2364

Ivezić, Ž., Richards, G., Hall, P., et al. 2004, in ASP Conf. Ser. 311, AGN

Physics with the Sloan Digital Sky Survey, ed. G. T. Richards \& P. B. Hall (San Francisco, CA: ASP), 347

Janknecht, E., Reimers, D., Lopez, S., \& Tytler, D. 2006, A\&A, 458, 427

Jiang, L., Fan, X., Ivezić, Ž., et al. 2007, ApJ, 656, 680

Jiang, L., Fan, X., Bian, F., et al. 2009, AJ, 138, 305

Just, D. W., Brandt, W. N., Shemmer, O., et al. 2007, ApJ, 665, 1004

Kaiser, C. R., \& Alexander, P. 1997, MNRAS, 286, 215

Kaspi, S., Brandt, W. N., \& Schneider, D. P. 2000, AJ, 119, 2031

Kellermann, K. I., Sramek, R., Schmidt, M., Shaffer, D. B., \& Green, R. 1989, AJ, 98, 1195

Kimball, A. E., Ivezić, Ž., Wiita, P. J., \& Schneider, D. P. 2011a, AJ, 141, 182

Kimball, A. E., Kellermann, K. I., Condon, J. J., Ivezić, Ž., \& Perley, R. A. 2011b, ApJ, 739, L29

Komatsu, E., Dunkley, J., Nolta, M. R., et al. 2009, ApJS, 180, 330

Krolik, J. H. 1999, Active Galactic Nuclei : from the Central Black Hole to the Galactic Environment (Princeton: Princeton University Press)

Latta, R. B., 1981, J. Am. Stat. Assoc., 76, 713

Lavalley, M., Isobe, T., Feigelson, E., 1992, in ASP Conf. Ser. 25, Astronomical Data Analysis Software and Systems I, ed. D. M. Worrall, C. Biemesderfer, \& J. Barnes (San Francisco, CA: ASP), 245

Lopez, L. A., Brandt, W. N., Vignali, C., et al. 2006, AJ, 131, 1914

Lyons, L. 1991, Data Analysis for Physical Science Students (Cambridge:

Cambridge Univ. Press)

Marshall, H. L., Gelbord, J. M., Schwartz, D. A., et al. 2011, ApJS, 193, 15

Massaro, F., Harris, D. E., \& Cheung, C. C. 2011, ApJS, 197, 24

McGreer, I. D., Helfand, D. J., \& White, R. L. 2009, AJ, 138, 1925

Meyer, E. T., Fossati, G., Georganopoulos, M., \& Lister, M. L. 2011, ApJ, 740, 98

Meyer, E. T., Fossati, G., Georganopoulos, M., \& Lister, M. L. 2012, ApJ, 752, L4

Migliori, G., Siemiginowska, A., \& Celotti, A. 2012, ApJ, 749, 107

Miller, B. P., Brandt, W. N., Schneider, D. P., et al. 2011, ApJ, 726, 20 (M11)

Momjian, E., Petric, A. O., \& Carilli, C. L. 2004, AJ, 127, 587

Mor, R., Netzer, H., Trakhtenbrot, B., Shemmer, O., \& Lira, P. 2012, ApJ, 749, L25

Morrison, R., \& McCammon, D. 1983, ApJ, 270, 119

Mullin, L. M., \& Hardcastle, M. J. 2009, MNRAS, 398, 1989
Nenkova, M., Sirocky, M. M., Nikutta, R., Ivezić, Ž., \& Elitzur, M. 2008, ApJ, 685, 160

Nieppola, E., Tornikoski, M., \& Valtaoja, E. 2006, A\&A, 445, 441

Nolan, P. L., Abdo, A. A., Ackermann, M., et al. 2012, ApJS, 199, 31

Nousek, J. A., \& Shue, D. R. 1989, ApJ, 342, 1207

Page, K. L., Reeves, J. N., O’Brien, P. T., \& Turner, M. J. L. 2005, MNRAS, 364, 195

Panter, B., Jimenez, R., Heavens, A. F., \& Charlot, S. 2007, MNRAS, 378, 1550

Perucho, M., Martí, J. M., \& Hanasz, M. 2005, A\&A, 443, 863

Reeves, J. N., \& Turner, M. J. L. 2000, MNRAS, 316, 234

Richards, G. T., Lacy, M., Storrie-Lombardi, L. J., et al. 2006, ApJS, 166, 470

Richards, G. T., Strauss, M. A., Fan, X., et al. 2006, AJ, 131, 2766

Richards, G. T., Kruczek, N. E., Gallagher, S. C., et al. 2011, AJ, 141, 167

Romani, R. W., Sowards-Emmerd, D., Greenhill, L., \& Michelson, P. 2004 ApJ, 610, L9

Romani, R. W. 2006, AJ, 132, 1959

Saez, C., Brandt, W. N., Shemmer, O., et al. 2011, ApJ, 738, 53

Sbarrato, T., Ghisellini, G., Maraschi, L., \& Colpi, M. 2012a, MNRAS, 421, 1764

Sbarrato, T., Ghisellini, G., Nardini, M., et al. 2012, MNRAS, 426, L91

Schneider, D. P., Hall, P. B., Richards, G. T., et al. 2007, AJ, 134, 102

Schneider, D. P., Richards, G. T., Hall, P. B., et al. 2010, AJ, 139, 2360

Schwartz, D. A. 2002, ApJ, 569, L23

Shang, Z., Brotherton, M. S., Wills, B. J., et al. 2011, ApJS, 196, 2 (S11)

Shaver, P. A., Wall, J. V., \& Kellermann, K. I. 1996, MNRAS, 278, L11

Shaw, M. S., Romani, R. W., Cotter, G., et al. 2012, ApJ, 748, 49

Shemmer, O., Brandt, W. N., Vignali, C., et al. 2005, ApJ, 630, 729

Shemmer, O., Brandt, W. N., Schneider, D. P., et al. 2006, ApJ, 644, 86

Shen, Y., Greene, J. E., Strauss, M. A., Richards, G. T., \& Schneider, D. P. 2008, ApJ, 680, 169

Shen, Y., Richards, G. T., Strauss, M. A., et al. 2011, ApJS, 194, 45

Siemiginowska, A., Smith, R. K., Aldcroft, T. L., et al. 2003, ApJ, 598, L15

Sikora, M., Madejski, G., Moderski, R., \& Poutanen, J. 1997, ApJ, 484, 108

Sikora, M., Stawarz, Ł., Moderski, R., Nalewajko, K., \& Madejski, G. M. 2009, ApJ, 704, 38

Singal, J., Petrosian, V., Lawrence, A., \& Stawarz, Ł. 2011, ApJ, 743, 104

Singal, J., Petrosian, V., Stawarz, L., \& Lawrence, A. 2012, arXiv:1207.3396 [astro-ph]

Skrutskie, M. F., Cutri, R. M., Stiening, R., et al. 2006, AJ, 131, 1163

Smail, I., Blundell, K. M., Lehmer, B. D., \& Alexander, D. M. 2012, ApJ, 760,132

Sokolov, A., Marscher, A. P., \& McHardy, I. M. 2004, ApJ, 613, 725

Songaila, A., \& Cowie, L. L. 2002, AJ, 123, 2183

Stark, A. A., Gammie, C. F., Wilson, R. W., et al. 1992, ApJS, 79, 77

Steffen, A. T., Strateva, I., Brandt, W. N., et al. 2006, AJ, 131, 2826

Tavecchio, F., Maraschi, L., Sambruna, R. M., \& Urry, C. M. 2000, ApJ, 544, L23

Vanden Berk, D. E., Richards, G. T., Bauer, A., et al. 2001, AJ, 122, 549

Vignali, C., Brandt, W. N., Fan, X., et al. 2001, AJ, 122, 2143

Vignali, C., Brandt, W. N., Schneider, D. P., Garmire, G. P., \& Kaspi, S. 2003a, AJ, 125, 418

Vignali, C., Brandt, W. N., Schneider, D. P., et al. 2003b, AJ, 125, 2876

Vignali, C., Brandt, W. N., Schneider, D. P., \& Kaspi, S. 2005, AJ, 129, 2519

Voges, W., Aschenbach, B., Boller, T., et al. 1999, A\&A, 349, 389

Volonteri, M., Haardt, F., Ghisellini, G., \& Della Ceca, R. 2011, MNRAS, 416, 216

Wang, R., Carilli, C. L., Wagg, J., et al. 2008, ApJ, 687, 848

Wang, R., Wagg, J., Carilli, C. L., et al. 2011a, AJ, 142, 101

Wang, Y., Knigge, C., Croston, J. H., \& Pavlovski, G. 2011b, MNRAS, 418, 1138

Weymann, R. J., Jannuzi, B. T., Lu, L., et al. 1998, ApJ, 506, 1

Wilkes, B. J., \& Elvis, M. 1987, ApJ, 323, 243

Wright, E. L., Eisenhardt, P. R. M., Mainzer, A. K., et al. 2010, AJ, 140, 1868

Worrall, D. M., Tananbaum, H., Giommi, P., \& Zamorani, G. 1987, ApJ, 313,596

Worsley, M. A., Fabian, A. C., Turner, A. K., Celotti, A., \& Iwasawa, K. 2004a, MNRAS, 350, 207

Worsley, M. A., Fabian, A. C., Celotti, A., \& Iwasawa, K. 2004b, MNRAS 350, L67

Worsley, M. A., Fabian, A. C., Pooley, G. G., \& Chandler, C. J. 2006, MNRAS, 368,844

Wu, J., Brandt, W. N., Hall, P. B., et al. 2011, ApJ, 736, 28

Wu, J., Brandt, W. N., Anderson, S. F., et al. 2012, ApJ, 747, 10

York, D. G., Adelman, J., Anderson, J. E., Jr., et al. 2000, AJ, 120, 1579

Yuan, W., Fabian, A. C., Celotti, A., \& Jonker, P. G. 2003, MNRAS, 346, L7

Yuan, W., Fabian, A. C., Celotti, A., McMahon, R. G., \& Matsuoka, M. 2005, MNRAS, 358, 432

Yuan, W., Fabian, A. C., Worsley, M. A., \& McMahon, R. G. 2006, MNRAS, 368, 985

Zamfir, S., Sulentic, J. W., \& Marziani, P. 2008, MNRAS, 387, 856

Zamorani, G., Henry, J. P., Maccacaro, T., et al. 1981, ApJ, 245, 357

Zickgraf, F.-J., Voges, W., Krautter, J., et al. 1997, A\&A, 323, L21 
TABLE 1

X-RAY OBSERVATION LOG

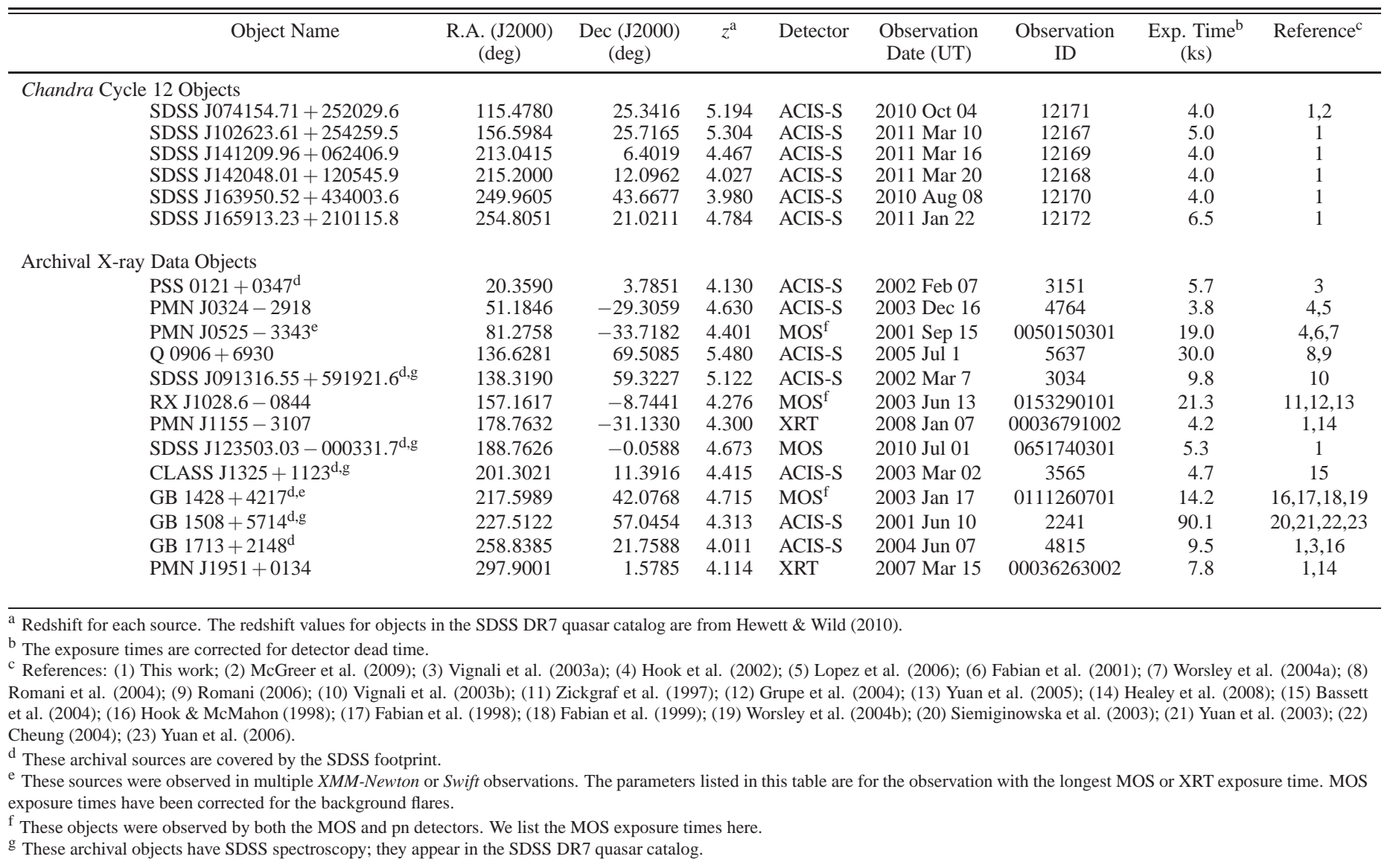

TABLE 2

HRLQS AT $z>4$ Without SENSITIVE X-RAY COVERAGE

\begin{tabular}{lrrrrrrr}
\hline \hline Object Name & $\begin{array}{c}\text { R.A. (J2000) } \\
(\mathrm{deg})\end{array}$ & $\begin{array}{c}\text { Dec (J2000) } \\
(\mathrm{deg})\end{array}$ & $z^{\mathrm{a}}$ & $m_{i}{ }^{\mathrm{b}}$ & $M_{i}$ & $\begin{array}{c}f_{1.4 \mathrm{GHz}}{ }^{\mathrm{c}} \\
(\mathrm{mJy})\end{array}$ & $\log R$ \\
\hline SDSS J003126.79+150739.5 & 7.8616 & 15.1276 & 4.297 & 20.0 & -27.2 & 41.0 & 2.824 \\
SDSS J030437.21+004653.5 & 46.1550 & 0.7815 & 4.260 & 20.2 & -27.1 & 20.0 & 3.259 \\
SDSS J081333.32+350810.8 & 123.3888 & 35.1363 & 4.945 & 19.2 & -28.2 & 20.0 & 2.663 \\
NVSS J105011-044254 & 162.5480 & -4.7150 & 4.270 & 23.4 & -23.8 & 6.0 & 3.338 \\
NVSS J112310-215405 & 170.7921 & -21.9016 & 4.110 & 22.9 & -24.1 & 48.0 & 4.041 \\
SDSS J123142.17+381658.9 & 187.9257 & 38.2830 & 4.128 & 20.1 & -26.9 & 25.0 & 2.777 \\
SDSS J123726.26+651724.4 & 189.3594 & 65.2901 & 4.300 & 20.5 & -26.6 & 24.0 & 2.785 \\
SDSS J124230.58+542257.3 & 190.6274 & 54.3826 & 4.766 & 19.7 & -27.7 & 19.0 & 2.678 \\
PMN J2134-0419 & 323.5501 & -4.3194 & 4.350 & 20.1 & -27.1 & 333.0 & 3.714 \\
SDSS J222032.50+002537.5 & 335.1354 & 0.4271 & 4.218 & 19.9 & -27.1 & 61.0 & 3.531 \\
PMN J2314+0201 & 348.7030 & 2.0309 & 4.110 & 20.2 & -26.9 & 117.0 & 3.308 \\
\hline
\end{tabular}

a The redshift values for SDSS objects, all of which appear in the SDSS DR7 quasar catalog, are from Hewett $\&$ Wild (2010).

${ }^{\mathrm{b}}$ The apparent $i$-band magnitude. For objects not in the SDSS footprint, the $i$-band magnitude was estimated

from their $R$-band magnitude by assuming a power law with a spectral index of $\alpha_{v}=-0.5$.

${ }^{c}$ Radio flux density at observed-frame 1.4 GHz from FIRST or NVSS. 
TABLE 3

X-ray Counts for Objects without Previously Published X-Ray Photometry

\begin{tabular}{cccccc}
\hline \hline Object Name & $\begin{array}{c}\text { Full Band } \\
(0.5-8.0 \mathrm{keV})^{\mathrm{a}}\end{array}$ & $\begin{array}{c}\text { Soft Band } \\
(0.5-2.0 \mathrm{keV})^{\mathrm{a}}\end{array}$ & $\begin{array}{c}\text { Hard Band } \\
(2.0-8.0 \mathrm{keV})^{\mathrm{a}}\end{array}$ & $\begin{array}{c}\text { Band } \\
\text { Ratio }^{\mathrm{b}}\end{array}$ & $\Gamma^{\mathrm{c}}$ \\
\hline Chandra Cycle 12 Objects & & & & & \\
SDSS J074154.71+252029.6 & $29.8_{-5.4}^{+6.5}$ & $17.8_{-4.2}^{+5.3}$ & $11.1_{-3.3}^{+4.4}$ & $0.62_{-0.23}^{+0.31}$ & $1.21_{-0.37}^{+0.44}$ \\
SDSS J102623.61+254259.5 & $59.3_{-7.7}^{+8.7}$ & $42.6_{-6.5}^{+7.6}$ & $14.3_{-3.7}^{+4.9}$ & $0.34_{-0.10}^{+0.13}$ & $1.73_{-0.31}^{+0.32}$ \\
SDSS J141209.96+062406.9 & $29.9_{-5.4}^{+6.5}$ & $18.9_{-4.3}^{+5.4}$ & $9.9_{-3.1}^{+4.3}$ & $0.52_{-0.20}^{+0.27}$ & $1.33_{-0.38}^{+0.43}$ \\
SDSS J142048.01+120545.9 & $48.9_{-7.0}^{+8.0}$ & $33.9_{-5.8}^{+6.9}$ & $13.1_{-3.6}^{+4.7}$ & $0.39_{-0.12}^{+0.16}$ & $1.61_{-0.32}^{+0.35}$ \\
SDSS J163950.52+434003.6 & $42.9_{-6.5}^{+7.6}$ & $25.8_{-5.0}^{+6.2}$ & $15.7_{-3.9}^{+5.1}$ & $0.61_{-0.19}^{+0.25}$ & $1.19_{-0.31}^{+0.34}$ \\
SDSS J165913.23+210115.8 & $43.6_{-6.6}^{+7.7}$ & $30.8_{-5.5}^{+6.6}$ & $11.1_{-3.3}^{+4.4}$ & $0.36_{-0.12}^{+0.16}$ & $1.75_{-0.36}^{+0.40}$ \\
& & & & & \\
Archival X-ray Data Objects & $12.1_{-3.4}^{+0.6}$ & $10.1_{-3.1}^{+4.3}$ & $<8.3$ & $<0.82$ & $>1.33$ \\
PMN J1155 -3107 & $25.9_{-8.1}^{+9.1}$ & $<18.3$ & $<15.9$ & $\ldots$ & $\ldots$ \\
SDSS J123503.03-000331.7 & $92.5_{-9.6}^{+10.7}$ & $63.2_{-7.9}^{+9.0}$ & $25.9_{-5.0}^{+6.2}$ & $0.41_{-0.09}^{+0.11}$ & $1.63_{-0.23}^{+0.24}$ \\
GB 1713 + 2148 & $45.2_{-6.7}^{+7.8}$ & $25.4_{-5.0}^{+6.1}$ & $20.7_{-4.5}^{+5.6}$ & $0.81_{-0.24}^{+0.30}$ & $1.54_{-0.30}^{+0.33}$ \\
PMN J1951+0134 & & & & & \\
\hline
\end{tabular}

${ }^{a}$ Errors on the X-ray counts were calculated using Poisson statistics corresponding to the $1 \sigma$ significance level according to Tables 1 and 2 of Gehrels (1986).

$\mathrm{b}$ The band ratio is defined here as the number of hard-band counts divided by the number of soft-band counts. The errors on the band ratio correspond to the $1 \sigma$ significance level and were calculated using equation (1.31) in $\S 1.7 .3$ of Lyons (1991). The band ratios for all of the Chandra objects observed in the same cycle can be directly compared with one another.

c The effective power-law photon indices were calculated using the PIMMS tool (version $3.9 k$ ). The effects of the quantum-efficiency decay over time at low energies of the ACIS detector were corrected for Chandra observed objects. The Chandra ACIS Cycle 5 response was used for GB 1713+2148, while the Chandra Cycle 12 response was used for the other objects. For J1155-3107 and J1235-0003 which do not have photon index estimation, we adopt a typical RLQ photon index value $\Gamma=1.6$ in the following analyses. 
TABLE 4

X-RAY, OPTICAL/UV, AND RADIO PROPERTIES

\begin{tabular}{|c|c|c|c|c|c|c|c|c|c|c|c|c|c|c|c|c|}
\hline $\begin{array}{l}\text { Object Name } \\
\text { (1) }\end{array}$ & $\begin{array}{l}m_{i}{ }^{\mathrm{a}} \\
(2)\end{array}$ & $\begin{array}{c}M_{i}^{\mathrm{b}} \\
(3)\end{array}$ & $\begin{array}{l}N_{\mathrm{H}} \\
(4)\end{array}$ & $\begin{array}{l}\text { Count } \\
\text { Rate }^{c} \\
(5)\end{array}$ & $\begin{array}{c}F_{X}{ }^{\mathrm{d}} \\
(6)\end{array}$ & $\begin{array}{l}f_{2 \mathrm{keV}^{\mathrm{e}}} \\
\text { (7) }\end{array}$ & $\begin{array}{c}\log L_{\mathrm{X}} \\
(2-10 \mathrm{keV})^{\mathrm{f}} \\
(8)\end{array}$ & $\begin{array}{l}\Gamma_{X} \mathrm{~g} \\
(9)\end{array}$ & $f_{2500} \AA^{\mathrm{h}}$ & $\begin{array}{c}\log L_{\mathrm{uv}} \\
(2500 \AA)^{\mathrm{i}} \\
\quad(11)\end{array}$ & $\begin{array}{l}\alpha_{\mathrm{r}}^{\mathrm{j}} \\
(12)\end{array}$ & $\begin{array}{c}\log L_{\mathrm{r}} \\
(5 \mathrm{GHz})^{\mathrm{k}} \\
\quad(13)\end{array}$ & $\begin{array}{c}\log R^{1} \\
(14)\end{array}$ & $\begin{array}{l}\alpha_{\mathrm{ox}} \\
(15)\end{array}$ & $\underset{(16)}{\Delta \alpha_{\mathrm{ox}, \mathrm{RQQ}}(\sigma)^{\mathrm{m}}}$ & $\begin{array}{c}\Delta \alpha_{\text {Ox, RLQ }}{ }^{\mathrm{n}} \\
\quad(17)\end{array}$ \\
\hline \multicolumn{17}{|l|}{ Chandra Cycle 12 Objects } \\
\hline SDSS J074154.71 + 252029.6 & 18.54 & -29.04 & 4.26 & $4.44_{-1.04}^{+1.33}$ & 1.89 & 6.80 & 45.20 & $1.21_{-0.37}^{+0.44}$ & 1.95 & 31.97 & $\ldots$ & 33.16 & 1.059 & -1.71 & $0.07(0.5)$ & -0.02 \\
\hline SDSS J102623.61+254259.5 & 20.03 & -27.50 & 1.80 & $8.55_{-1.30}^{+1.52}$ & 3.51 & 24.03 & 45.76 & $1.73_{-0.31}^{+0.32}$ & 0.62 & 31.49 & -0.38 & 35.15 & 3.542 & -1.31 & $0.39(2.7)$ & 0.07 \\
\hline SDSS J141209.96+062406.9 & 19.44 & -27.74 & 2.11 & $4.73_{-1.08}^{+1.30}$ & 1.93 & 7.73 & 45.15 & $1.33_{-0.38}^{+0.43}$ & 0.65 & 31.40 & $\ldots$ & 34.36 & 2.698 & -1.51 & $0.18(1.2)$ & -0.06 \\
\hline SDSS J142048.01+ 120545.9 & 19.80 & -27.18 & 1.72 & $8.47_{-1.45}^{+1.08}$ & 3.45 & 17.84 & 45.44 & $1.61_{-0.32}^{+0.35}$ & 0.56 & 31.26 & -0.36 & 34.55 & 3.052 & -1.34 & $0.33(2.3)$ & $0.0 \frac{\pi}{8}$ \\
\hline SDSS J163950.52+434003.6 & 17.78 & -29.13 & 1.36 & $6.45_{-1.26}^{+1.54}$ & 2.57 & 8.67 & 45.13 & $1.19_{-0.31}^{+0.34}$ & 4.29 & 32.14 & -0.32 & 33.93 & 1.621 & -1.80 & $-0.01(0.1)$ & -0 \\
\hline SDSS J165913.23 + 210115.8 & 20.26 & -27.17 & 5.47 & $4.77_{-0.85}^{+1.02}$ & 2.15 & 14.12 & 45.34 & $1.75_{-0.36}^{+0.40}$ & 0.60 & 31.40 & $\cdots$ & 34.25 & 2.557 & -1.39 & $0.30(2.1)$ & $0.0 \overline{\bar{z}}$ \\
\hline \multicolumn{17}{|l|}{ Archival X-ray Data Objects } \\
\hline PSS $0121+0347$ & 18.57 & -28.44 & 3.19 & $10.30_{-1.30}^{+1.60}$ & 3.19 & 26.78 & 45.64 & $2.10_{-0.30}^{+0.40}$ & 1.77 & 31.78 & -0.33 & 34.47 & 2.573 & -1.47 & $0.28(1.9)$ & 0.04 \\
\hline PMN J0324-2918 & 18.65 & -28.61 & 1.19 & $13.70_{-1.90}^{+2.20}$ & 5.83 & 39.70 & 45.89 & $1.80_{-0.40}^{+0.40}$ & 1.73 & 31.84 & +0.30 & 34.92 & 2.953 & -1.40 & $0.35(2.4)$ & $0 . \overline{8}$ \\
\hline PMN J0525 - 3343 & 18.63 & -28.52 & 2.19 & $\ldots^{-1.90}$ & 27.70 & 159.40 & 46.46 & $1.67_{-0.02}^{+0.02}$ & 1.73 & 31.81 & +0.06 & 34.84 & 2.903 & -1.17 & $0.58(4.0)$ & $0.3 \frac{1}{t}$ \\
\hline Q $0906+6930$ & 19.85 & -27.76 & 3.64 & $10.72_{-0.60}^{+0.63}$ & 4.16 & 24.78 & 45.80 & $1.6_{-0.1}^{+0.1}$ & 0.61 & 31.51 & +0.17 & 34.64 & 3.008 & -1.31 & $0.40(2.7)$ & 0.12 \\
\hline SDSS J091316.55 + 591921.6 & 20.39 & -27.03 & 3.85 & $0.51_{-0.29}^{+0.34}$ & 0.16 & 0.95 & 44.34 & $>0.71$ & 0.36 & 31.23 & -0.67 & 34.07 & 2.717 & -1.76 & $-0.09(0.6)$ & -0.0 \\
\hline RX J1028.6 - 0844 & 19.14 & -27.95 & 4.60 & $\ldots$ & 38.14 & 179.40 & 46.49 & $1.40_{-0.03}^{+0.03}$ & 1.26 & 31.65 & -0.30 & 35.03 & 3.329 & -1.09 & $0.63(4.3)$ & 0.39 \\
\hline PMN J1155-3107 & 19.28 & -27.90 & 6.04 & $\ldots$ & 5.63 & 29.79 & 45.34 & $>1.33$ & 1.01 & 31.56 & +0.53 & 34.37 & 2.727 & -1.36 & $0.36(2.4)$ & 0.1 \\
\hline SDSS J123503.03 - 000331.7 & 20.10 & -27.20 & 1.90 & $\ldots$ & 2.15 & 10.57 & 44.96 & $\ldots$ & 0.16 & 30.81 & $\ldots$ & 33.87 & 3.051 & -1.22 & $0.39(1.9)$ & $0.1 \frac{6}{6}$ \\
\hline CLASS J1325 + 1123 & 19.18 & -28.01 & 1.93 & $5.04_{-1.05}^{+1.26}$ & 1.62 & 10.71 & 45.29 & $1.80_{-0.40}^{+0.50}$ & 1.03 & 31.59 & -0.09 & 34.43 & 2.722 & -1.53 & $0.19(1.3)$ & $-0 . \mathbb{P S}$ \\
\hline GB $1428+4217$ & 19.10 & -28.18 & 1.39 & $x^{-1.05}$ & 70.07 & 447.30 & 46.95 & $1.73_{-0.03}^{+0.03}$ & 1.15 & 31.68 & +0.37 & 34.87 & 3.065 & -0.93 & $0.80(5.5)$ & 0.52 \\
\hline GB $1508+5714$ & 19.92 & -27.16 & 1.46 & $42.06_{-0.69}^{+0.70}$ & 15.08 & 76.68 & 46.13 & $1.55_{-0.05}^{+0.03}$ & 0.24 & 30.94 & +0.13 & 34.93 & 3.871 & -0.96 & $0.67(3.4)$ & 0.34 \\
\hline GB $1713+2148$ & 21.42 & -25.53 & 5.05 & $6.63_{-0.83}^{+0.94}$ & 2.36 & 12.40 & 45.29 & $1.63_{-0.23}^{+0.05}$ & 0.13 & 30.61 & -0.30 & 35.11 & 4.501 & -1.16 & $0.42(2.1)$ & 0.025 \\
\hline PMN J1951 + 0134 & 19.69 & -27.40 & 16.42 & $\ldots$ & 8.78 & 42.79 & 45.51 & $1.54_{-0.30}^{+0.33}$ & 0.69 & 31.36 & +0.24 & 34.48 & 3.036 & -1.23 & $0.45(3.1)$ & 0.20 \\
\hline
\end{tabular}

The absolute $i$-band magnitude, corrected for Galactic extinction.

The count rate of the Chandra-observed sources in the observed-frame soft X-ray hand $(0.5-2.0 \mathrm{keV})$, in units of $10^{-3} \mathrm{~s}^{-1}$.

$\mathrm{d}$ The Galactic absorption-corrected observed-frame fux between $0.5-20 \mathrm{keV}$ in units of $10^{-14} \mathrm{egs} \mathrm{s}^{-2} \mathrm{~s}^{-1}$.

$e^{e}$ The flux density at rest-frame $2 \mathrm{keV}$, in units of $10^{-32} \mathrm{ergs} \mathrm{cm}^{-2} \mathrm{~s}^{-1} \mathrm{~Hz}^{-1}$.

The logarithm of the X-ray luminosity in the rest-frame $2-10 \mathrm{keV}$ band, corrected for Galactic absorption

$\mathrm{g}$ The X-ray power-law photon index (also see Table 3 .

The flux density at rest-frame $2500 \AA$ in units of $10^{-27} \mathrm{ergs} \mathrm{cm}^{-2} \mathrm{~s}^{-1} \mathrm{~Hz}^{-1}$.

${ }^{1}$ The logarithm of the monochromatic UV luminosity at rest-frame $2500 \AA$.

j The radio spectral index $\alpha$ between observed-frame 1.4-5 GHz, defined as $f_{v} \propto v^{\alpha}$.

${ }^{\mathrm{k}}$ The logarithm of monochromatic radio luminosity at rest-frame $5 \mathrm{GH}$

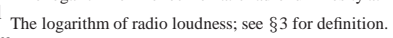

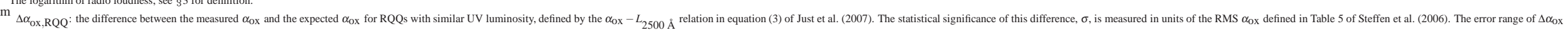

is $\sim 0.003-0.07$

$\Delta \alpha_{0 x, R L Q}$ : the difference between the measured $\alpha_{0 x}$ and the expected $\alpha_{0 x}$ for RLQs with similar UV and radio luminosities, defined by the $L_{2} \mathrm{keV}-L_{2500} \AA-L_{5} \mathrm{GHz}$ relation in Table 7 of Miller et al. (2011) for their full sample 
TABLE 5

Results of Peto-Prentice Tests

\begin{tabular}{|c|c|c|c|c|c|c|}
\hline Parameter & $\begin{array}{c}\text { No. of } \\
\text { Our HRLQs }\end{array}$ & $\begin{array}{l}\text { No. of Comparison Objects } \\
\text { (No. of X-ray Limits) }\end{array}$ & $\begin{array}{c}\text { Mean } \\
\text { (Our HRLQs) }\end{array}$ & $\begin{array}{c}\text { Mean } \\
\text { (Comparison Objects) }\end{array}$ & Statistic & $\begin{array}{l}\text { Null-hypothesis } \\
\text { Probability }\end{array}$ \\
\hline$\Delta \alpha_{\mathrm{OX}, \mathrm{RQQ}}$ & 12 & $283(14)$ & $0.435 \pm 0.054$ & $0.251 \pm 0.008$ & 3.844 & $1.21 \times 10^{-4}$ \\
\hline$\Delta \alpha_{\mathrm{Ox}, \mathrm{RLQ}}$ & 12 & $283(14)$ & $0.167 \pm 0.049$ & $0.005 \pm 0.007$ & 3.814 & $1.37 \times 10^{-4}$ \\
\hline$\Delta \alpha_{\mathrm{ox}, \mathrm{RQQ}}$ (Worrall et al.'s Objects) & $8^{\mathrm{a}}$ & $81(7)$ & $0.475 \pm 0.065$ & $0.302 \pm 0.018$ & 2.682 & $7.32 \times 10^{-3}$ \\
\hline$\Delta \alpha_{\mathrm{OX}, \mathrm{RLQ}}$ (Worrall et al.'s Objects) & 8 & $81(7)$ & $0.204 \pm 0.059$ & $0.014 \pm 0.016$ & 3.181 & $1.47 \times 10^{-3}$ \\
\hline$\Delta \alpha_{\mathrm{ox}, \mathrm{RQQ}}($ FIRST-selected $)$ & 8 & $70(0)$ & $0.475 \pm 0.065$ & $0.241 \pm 0.011$ & 3.892 & $9.94 \times 10^{-5}$ \\
\hline$\Delta \alpha_{\mathrm{ox}, \mathrm{RLQ}}(\mathrm{FIRST}$-selected $)$ & 8 & $70(0)$ & $0.205 \pm 0.059$ & $0.002 \pm 0.011$ & 3.814 & $1.37 \times 10^{-4}$ \\
\hline$\Delta \alpha_{\mathrm{OX}, \mathrm{RQQ}}\left(\log L_{5 \mathrm{GHz}}>34\right)^{\mathrm{b}}$ & 12 & $133(7)$ & $0.435 \pm 0.054$ & $0.294 \pm 0.012$ & 2.668 & $7.63 \times 10^{-3}$ \\
\hline$\Delta \alpha_{\mathrm{Ox}, \mathrm{RLQ}}\left(\log L_{5 \mathrm{GHz}}>34\right)$ & 12 & $133(7)$ & $0.167 \pm 0.049$ & $-0.001 \pm 0.011$ & 3.628 & $2.86 \times 10^{-4}$ \\
\hline$\Delta \alpha_{\mathrm{OX}, \mathrm{RQQ}}\left(\log L_{2500} \AA>30.9\right)^{\mathrm{b}}$ & 12 & $90(6)$ & $0.435 \pm 0.054$ & $0.305 \pm 0.016$ & 2.363 & $1.81 \times 10^{-2}$ \\
\hline$\Delta \alpha_{\mathrm{Ox}, \mathrm{RLQ}}\left(\log L_{2500 \AA}>30.9\right)$ & 12 & $90(6)$ & $0.167 \pm 0.049$ & $0.018 \pm 0.013$ & 3.126 & $1.77 \times 10^{-3}$ \\
\hline$\Delta \alpha_{\mathrm{OX}, \mathrm{RQQ}}\left(\alpha_{\mathrm{r}}>-0.5\right)$ & $11^{\mathrm{c}}$ & $90(2)$ & $0.458 \pm 0.054$ & $0.261 \pm 0.014$ & 3.782 & $1.56 \times 10^{-4}$ \\
\hline$\Delta \alpha_{\mathrm{Ox}, \mathrm{RLQ}}\left(\alpha_{\mathrm{r}}>-0.5\right)$ & 11 & $90(2)$ & $0.188 \pm 0.049$ & $0.029 \pm 0.013$ & 3.587 & $3.35 \times 10^{-4}$ \\
\hline
\end{tabular}

NOTE. - For the detailed definition of the Peto-Prentice test statistic see Feigelson \& Nelson (1985). The null-hypothesis probability was calculated from the test statistic based on a Gaussian distribution, e.g., $1-P_{G}=1.21 \times 10^{-4}$, where $P_{G}$ is the cumulative Gaussian probability at $3.844 \sigma$.

${ }^{a}$ The number of radio-selected objects in our $z>4$ HRLQ sample that satisfy $m_{i}<20$. One object, GB $1713+2148$, has $m_{i}>20$, and thus is excluded from the two-sample tests.

b The range of radio luminosity $L_{5} \mathrm{GHz}>34$ and optical/UV luminosity $L_{2500} \AA>30.9$ were determined based on our $z>4$ HRLQs with $m_{i}<20$. Thus all 12 of our HRLQs surviving this $m_{i}$ cut are included in these tests.

${ }^{\mathrm{c}}$ All of our $12 z>4$ HRLQs with $m_{i}<20$ are FSRQs except for J1412+0624 which does not have available $\alpha_{\mathrm{r}}$ measurement.

TABLE 6

Results of Peto-Prentice Tests For Additional Redshift Ranges

\begin{tabular}{cccc}
\hline \hline Sample & $\begin{array}{c}\text { No. of HRLQs } \\
\text { (No. of X-ray Limits) }\end{array}$ & Mean $\left(\Delta \alpha_{\text {ox }, \mathrm{RQQ}}\right)$ & Mean $\left(\Delta \alpha_{\text {ox }, \mathrm{RLQ}}\right)$ \\
\hline $3 \leqslant z<4$ & $9(1)$ & $0.468 \pm 0.056$ & $0.167 \pm 0.046$ \\
$z<3$ & $274(13)$ & $0.244 \pm 0.008$ & $0.000 \pm 0.007$ \\
\hline Test Statistic & & 4.954 & 5.428 \\
Null-hypothesis Probability & & $7.27 \times 10^{-7}$ & $5.70 \times 10^{-8}$ \\
\hline \hline & & & \\
$2 \leqslant z<3$ & $39(4)$ & $0.249 \pm 0.023$ & $-0.030 \pm 0.019$ \\
$z<2$ & $235(9)$ & $0.243 \pm 0.009$ & $0.005 \pm 0.008$ \\
\hline Test Statistic & & 0.176 & 1.981 \\
Null-hypothesis Probability & & 0.861 & 0.048 \\
\hline \hline$z \geqslant 3$ & $21(1)$ & $0.450 \pm 0.039$ & $0.167 \pm 0.034$ \\
$z<3$ & $274(13)$ & $0.244 \pm 0.008$ & $0.000 \pm 0.007$ \\
\hline Test Statistic & & 5.905 & 5.726 \\
& & $3.53 \times 10^{-9}$ & $1.03 \times 10^{-8}$ \\
Null-hypothesis Probability & & & \\
\hline
\end{tabular}

Note. - For the detailed definition of the Peto-Prentice test statistic see Feigelson \& Nelson (1985). The null-hypothesis probability was calculated from the test statistic based on a Gaussian distribution, e.g., $1-P_{G}=7.27 \times 10^{-7}$, where $P_{G}$ is the cumulative Gaussian probability at $4.954 \sigma$. 
TABLE 7

X-Ray Properties of $z>4$ HRLQs Derived From the ObSerVed-Frame Ultrasoft Band (0.3-1.0 KeV)

\begin{tabular}{|c|c|c|c|c|c|c|c|}
\hline Object Name & Count Rate ${ }^{\mathrm{a}}$ & $F_{\mathrm{X}, \mathrm{US}}{ }^{\mathrm{b}}$ & $f_{2 \mathrm{keV}, \mathrm{US}^{\mathrm{c}}}$ & $\alpha_{\mathrm{ox}, \mathrm{US}}{ }^{\mathrm{d}}$ & $\Delta \alpha_{\mathrm{OX}, \mathrm{RQQ}, \mathrm{US}}^{\mathrm{e}}$ & $\Delta \alpha_{\text {ox,RLQ,US }}{ }^{\mathrm{f}}$ & $\alpha_{\mathrm{ox}, \mathrm{US}}-\alpha_{\mathrm{ox}}{ }^{\mathrm{g}}$ \\
\hline \multicolumn{8}{|l|}{ Chandra Cycle 12 Objects } \\
\hline SDSS J141209.96+062406.9 & $1.32_{-0.55}^{+0.86}$ & 0.81 & 5.66 & -1.56 & 0.13 & -0.11 & -0.05 \\
\hline SDSS J142048.01 + 120545.9 & $3.89_{-0.97}^{+1.26}$ & 2.48 & 18.75 & -1.33 & 0.34 & 0.07 & 0.01 \\
\hline \multicolumn{8}{|l|}{ Archival X-ray Data Objects } \\
\hline PSS $0121+0347$ & $5.93_{-1.02}^{+1.21}$ & 2.02 & 18.41 & -1.53 & 0.22 & -0.02 & -0.06 \\
\hline PMN J0324-2918 & $6.62_{-1.35}^{+1.06}$ & 4.37 & 38.69 & -1.40 & 0.35 & 0.08 & 0.00 \\
\hline PMN J0525 - 3343 & $34.33_{-0.85}^{+0.87}$ & 22.07 & 178.82 & -1.15 & 0.60 & 0.33 & 0.02 \\
\hline Q $0906+6930$ & $5.32_{-0.43}^{+0.47}$ & 3.55 & 31.09 & -1.26 & 0.45 & 0.18 & 0.05 \\
\hline RX J1028.6 - 0844 & $21.80_{-0.70}^{+0.73}$ & 16.25 & 118.41 & -1.16 & 0.56 & 0.27 & -0.07 \\
\hline CLASS J1325 + 1123 & $2.01_{-0.64}^{+0.89}$ & 0.75 & 6.43 & -1.61 & 0.11 & -0.13 & -0.08 \\
\hline GB $1428+4217$ & $53.55_{-1.37}^{+1.44}$ & 32.45 & 281.56 & -1.00 & 0.73 & 0.45 & -0.07 \\
\hline GB $1508+5714$ & $22.33_{-0.50}^{+0.51}$ & 6.37 & 48.61 & -1.03 & 0.60 & 0.27 & -0.07 \\
\hline
\end{tabular}

NOTE. - The objects included in this table are our $z>4$ HRLQs with $m_{i}<20$, i.e., those $z>4$ HRLQs used in the two-sample analyses in 4.1 except for the two Swift sources (PMN J1155-3107 and PMN J1951+0134).

a The Chandra or XMM-Newton count rate in the observed-frame ultrasoft X-ray band (0.3-1.0 keV), in units of $10^{-3} \mathrm{~s}^{-1}$.

$\mathrm{b}$ The Galactic-absorption corrected flux in the observed-frame ultrasoft X-ray band, in units of $10^{-14} \mathrm{erg} \mathrm{cm}^{-2} \mathrm{~s}^{-1}$.

c The X-ray flux density at rest-frame $2 \mathrm{keV}$ derived from the ultrasoft band count rate, in units of $10^{-32} \mathrm{erg} \mathrm{cm}^{-2} \mathrm{~s}^{-1} \mathrm{~Hz}^{-1}$.

$\mathrm{d}$ The $\alpha_{\mathrm{ox}}$ values obtained from the ultrasoft X-ray band data.

e The $\Delta \alpha_{\text {ox,RQQ }}$ values obtained from the ultrasoft X-ray band data.

f The $\Delta \alpha_{\text {ox }, \text { RLQ }}$ values obtained from the ultrasoft X-ray band data.

$\mathrm{g}$ The difference between the $\alpha_{\mathrm{ox} \text {,Us }}$ values in this table and the $\alpha_{\mathrm{ox}}$ values in Column (15) of Table 4 which are obtained from the observed-frame soft band (0.5-2.0 keV) data. The X-ray spectral curvature discussed in 4.1 .3 is expected to result in negative values for this difference.

TABLE 8

EMISSION-LINE REW MEASUREMENTS

\begin{tabular}{cccc}
\hline \hline Object Name & MJD $^{\mathrm{a}}$ & REW(Ly $\alpha+\mathrm{N} \mathrm{v})$ & REW(C IV) \\
\hline Chandra Cycle 12 Objects & & & \\
SDSS J102623.61+254259.5 & 53734 & 41.6 & $\ldots$ \\
SDSS J141209.96+062406.9 & 53504 & 2.4 & 7.1 \\
SDSS J142048.01+120545.9 & 53885 & 55.0 & 52.7 \\
SDSS J163950.52+434003.6 & 52051 & 23.3 & 7.8 \\
SDSS J165913.23+210115.8 & 52913 & 17.9 & 6.4 \\
& & & \\
Archival X-ray Data Objects & 53328 & 81.1 & 33.5 \\
PSS 0121+0347 & 51907 & 110.9 & $\ldots$ \\
SDSS J091316.55+591921.6 & 39.5 & 54.1 \\
SDSS J123503.03-000331.7 & 51941 & 75.8 & 63.4 \\
CLASS J1325+1123 & 53148 & 17.1 & 12.9 \\
GB 1428+4217 & 50283 & 60.1 & 47.0 \\
GB 1508 +5714 & 52079 & 107.0 & 127.7 \\
GB 1713 +2148 & 50280 & & \\
\hline
\end{tabular}

NotE. - All REW values are in units of $\AA$.

a The MJD (Modified Julian Date) listed here is the date of optical/UV spectroscopy.

b Two objects (J0913+5919 and J1026+2542) do not have C IV coverage in their SDSS spectra because of their high redshifts.

${ }^{c}$ Reference for emission-line REW measurements: Vignali et al. (2003a).

${ }^{\mathrm{d}}$ Reference for emission-line REW measurements: Hook \& McMahon (1998). 
TABLE 9

Non-Thermal Dominance (NTD) CALCUlation

\begin{tabular}{ccccc}
\hline \hline Object Name & $\begin{array}{c}\log L_{\mathrm{C}} \mathrm{IV} \\
\left(\mathrm{erg} \mathrm{s}^{-1}\right)\end{array}$ & $\begin{array}{c}\log v L_{1350, \text { obs }} \\
\left(\mathrm{erg} \mathrm{s}^{-1}\right)\end{array}$ & $\begin{array}{c}\log v L_{1350, \text { pred }} \\
\left(\mathrm{erg} \mathrm{s}^{-1}\right)\end{array}$ & NTD \\
\hline Chandra Cycle 12 Objects & & & & \\
SDSS J141209.96+062406.9 & 44.289 & 46.670 & 45.881 & 6.150 \\
SDSS J142048.01+120545.9 & 44.968 & 46.465 & 46.467 & 0.995 \\
SDSS J163950.52+434003.6 & 44.946 & 47.255 & 46.448 & 6.402 \\
SDSS J165913.23+210115.8 & 44.121 & 46.516 & 45.736 & 6.021 \\
& & & & \\
Archival X-ray Data Objects & & & & \\
SDSS J123503.03 - 000331.7 & 44.597 & 46.100 & 46.148 & 0.896 \\
CLASS J1325+1123 & 45.425 & 46.860 & 46.861 & 0.997 \\
GB 1508+5714 & 44.873 & 46.503 & 46.385 & 1.312 \\
& & & & \\
\hline
\end{tabular}

TABLE 10

CORRELATION ANALYSIS RESUlTS

\begin{tabular}{|c|c|c|c|c|c|c|}
\hline & \multicolumn{3}{|c|}{$\operatorname{REW}(\operatorname{Ly} \alpha+\mathrm{N} v)$} & \multicolumn{3}{|c|}{$\mathrm{REW}(\mathrm{C}$ IV $)$} \\
\hline & \multirow[t]{2}{*}{$N^{a}$} & \multicolumn{2}{|c|}{ Spearman } & \multirow[t]{2}{*}{$N^{a}$} & \multicolumn{2}{|c|}{ Spearman } \\
\hline & & $r_{S}$ & $1-P_{S}$ & & $r_{S}$ & $1-P_{S}$ \\
\hline$\Delta \alpha_{\mathrm{OX}, \mathrm{ROQ}}$ & 32 & -0.19 & $71.0 \%$ & 169 & 0.14 & $93.3 \%$ \\
\hline$\Delta \alpha_{\mathrm{OX}, \mathrm{RLQ}}$ & 32 & -0.21 & $75.0 \%$ & 169 & 0.08 & $69.0 \%$ \\
\hline $\log R$ & 298 & 0.14 & $98.0 \%$ & 3536 & 0.30 & $>99.99 \%$ \\
\hline
\end{tabular}

${ }^{\mathrm{a}} \mathrm{N}$ is the sample size.

TABLE 11

Joint X-RAY Spectral Analysis Results

\begin{tabular}{cccccccc}
\hline \hline & \multicolumn{2}{c}{$\begin{array}{c}\text { Power Law } \\
\text { with Galactic Absorption }\end{array}$} & & \multicolumn{2}{c}{$\begin{array}{c}\text { Power Law } \\
\text { with Galactic and Intrinsic Absorption }\end{array}$} \\
\cline { 2 - 3 } Object Name & $\Gamma_{\mathrm{X}}$ & $C / n^{\mathrm{a}}$ & & $\Gamma_{\mathrm{X}}$ & $N_{H}\left(10^{22} \mathrm{~cm}^{-2}\right)$ & $C / n^{\mathrm{a}}$ \\
\hline $\mathrm{J} 1026 / \mathrm{J} 1412 / \mathrm{J} 1420 / \mathrm{J} 1659^{\mathrm{b}}$ & $1.59_{-0.24}^{+0.24}$ & $122.47 / 148$ & & $1.60_{-0.24}^{+0.40}$ & $<9.50$ & $122.47 / 148$ \\
$\mathrm{~J} 0741 / \mathrm{J} 1659^{\mathrm{c}}$ & $1.34_{-0.37}^{+0.39}$ & $46.87 / 66$ & & $1.34_{-0.36}^{+0.49}$ & $<12.41$ & $46.87 / 66$ \\
& & & & & & & \\
\hline
\end{tabular}

${ }^{\text {a }} C$ is the $C$-statistic, while $n$ is the total number of spectral bins.

${ }^{\mathrm{b}}$ Our Chandra Cycle 12 targets with $\log R>2.5$. The names are in the format of 'Jhhmm' for brevity.

c Our Chandra Cycle 12 targets that are moderately radio loud. The names are in the format of 'Jhhmm' for brevity. 
TABLE 12

UPPER LIMITS UPON $\gamma$-RAY FLUX AND LUMINOSITY FROM THE Fermi LAT

\begin{tabular}{|c|c|c|c|c|}
\hline Object Name & $\begin{array}{l}\text { Photon Flux Limit }{ }^{\mathrm{a}} \\
\left(10^{-9} \text { photons } \mathrm{cm}^{-2} \mathrm{~s}^{-1}\right)\end{array}$ & $\begin{array}{l}\text { Energy Flux Limit }{ }^{\mathrm{b}} \\
\left(10^{-12} \mathrm{erg} \mathrm{cm}^{-2} \mathrm{~s}^{-1}\right)\end{array}$ & $\begin{array}{c}\text { Flux Density Limit } \\
\left(10^{-35} \mathrm{erg} \mathrm{cm}^{-2} \mathrm{~s}^{-1} \mathrm{~Hz}^{-1}\right)\end{array}$ & $\begin{array}{l}\log \left(v L_{1 \mathrm{GeV}}\right) \operatorname{Limit}^{\mathrm{d}} \\
\left(\mathrm{erg} \mathrm{s}^{-1} \mathrm{~Hz}^{-1}\right)\end{array}$ \\
\hline \multicolumn{5}{|l|}{ Chandra Cycle 12 Objects } \\
\hline SDSS J074154.71+252029.6 & 4.5 & 2.4 & 2.1 & 47.4 \\
\hline SDSS J102623.61+254259.5 & 4.5 & 2.4 & 2.2 & 47.4 \\
\hline SDSS J141209.96+062406.9 & 5.0 & 2.6 & 2.0 & 47.3 \\
\hline SDSS J142048.01+120545.9 & 5.0 & 2.6 & 1.8 & 47.1 \\
\hline SDSS J163950.52+434003.6 & 4.5 & 2.4 & 1.6 & 47.1 \\
\hline SDSS J165913.23 + 210115.8 & 4.5 & 2.4 & 1.9 & 47.3 \\
\hline \multicolumn{5}{|l|}{ Archival X-ray Data Objects } \\
\hline PSS $0121+0347$ & 4.5 & 2.4 & 1.6 & 47.1 \\
\hline PMN J0324-2918 & 4.5 & 2.4 & 1.9 & 47.3 \\
\hline PMN J0525 - 3343 & 4.5 & 2.4 & 1.8 & 47.2 \\
\hline Q $0906+6930$ & 3.5 & 1.8 & 1.8 & 47.4 \\
\hline SDSS J091316.55+591921.6 & 4.0 & 2.1 & 1.9 & 47.3 \\
\hline RX J1028.6- 0844 & 5.0 & 2.6 & 1.9 & 47.2 \\
\hline PMN J1155-3107 & 5.0 & 2.6 & 1.9 & 47.2 \\
\hline SDSS J123503.03-000331.7 & 5.0 & 2.6 & 2.1 & 47.3 \\
\hline CLASS J1325 + 1123 & 5.0 & 2.6 & 2.0 & 47.3 \\
\hline GB $1428+4217$ & 4.0 & 2.1 & 1.7 & 47.2 \\
\hline GB $1508+5714$ & 4.0 & 2.1 & 1.5 & 47.1 \\
\hline GB $1713+2148$ & 5.0 & 2.6 & 1.8 & 47.1 \\
\hline PMN J1951 + 0134 & 7.0 & 3.7 & 2.5 & 47.3 \\
\hline
\end{tabular}

${ }^{a}$ The upper limit upon the Fermi LAT photon flux between observed-frame $100 \mathrm{MeV}-100 \mathrm{GeV}$.

${ }^{b}$ The upper limit upon the $\gamma$-ray energy flux between observed-frame $100 \mathrm{MeV}-100 \mathrm{GeV}$.

${ }^{c}$ The upper limit upon the $\gamma$-ray flux density at rest-frame $1 \mathrm{GeV}$.

$\mathrm{d}$ The upper limit upon the monochromatic $\gamma$-ray luminosity at rest-frame $1 \mathrm{GeV}$.

TABLE 13

Measurements of Black-Hole Mass and Eddington Ratio

\begin{tabular}{crc}
\hline \hline Object Name & $\log M_{\mathrm{BH}}\left(M_{\odot}\right)$ & $\log \left(L / L_{\mathrm{Edd}}\right)$ \\
\hline Chandra Cycle 12 Objects & & \\
SDSS J141209.96+062406.9 & $9.859 \pm 1.218$ & -0.708 \\
SDSS J142048.01+120545.9 & $9.284 \pm 0.180$ & -0.338 \\
SDSS J163950.52+434003.6 & $10.628 \pm 0.168$ & -0.893 \\
& & \\
Archival X-ray Data Objects & & \\
SDSS J123503.03-000331.7 & $9.182 \pm 0.727$ & -0.602 \\
CLASS J1325+1123 & $9.455 \pm 0.071$ & -0.115 \\
GB 1508+5714 & $8.517 \pm 0.388$ & 0.467 \\
& & \\
\hline
\end{tabular}




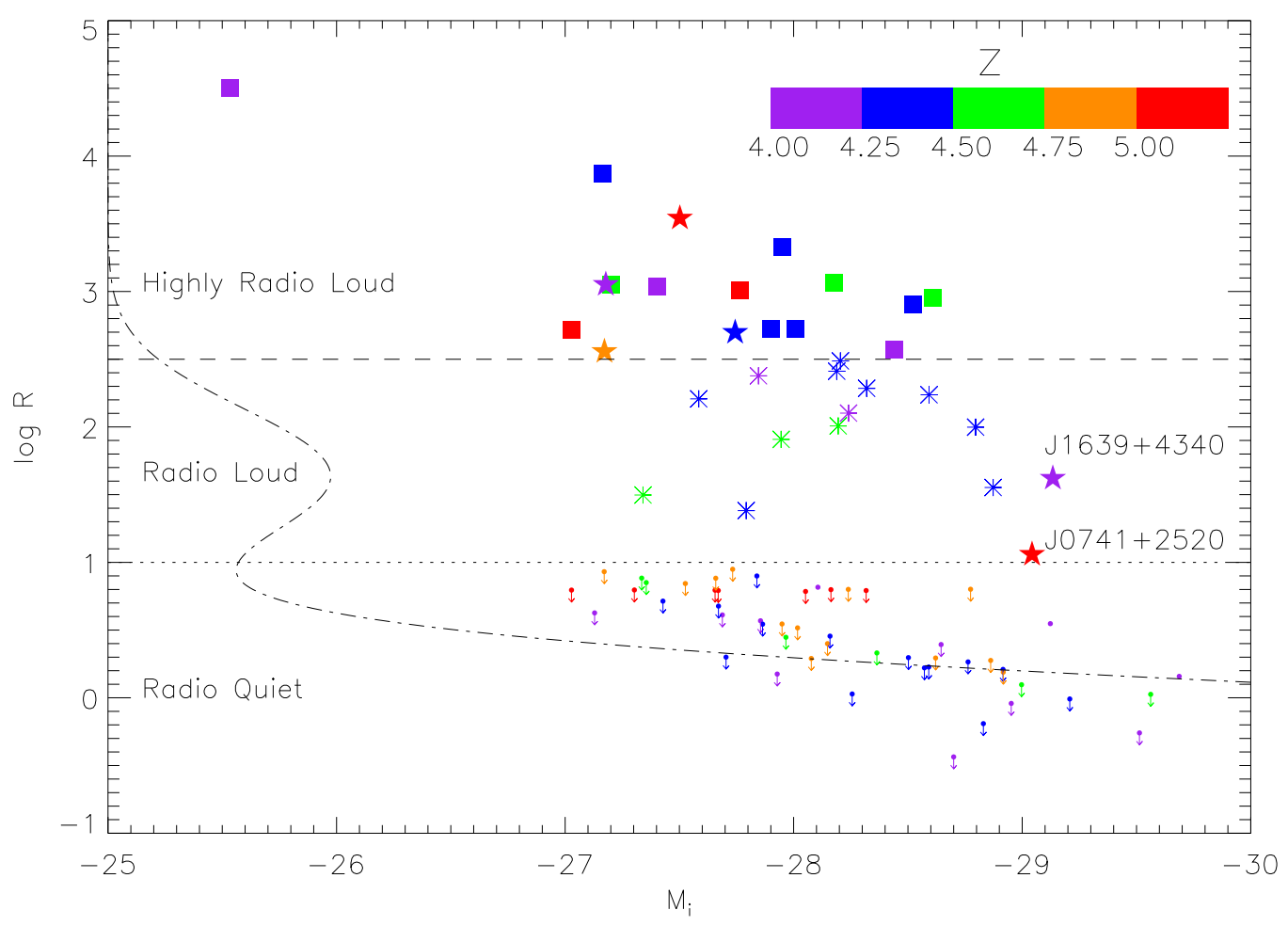

FIG. 1.- The distribution of our sample of RLQs in the plane of $M_{i}$ (SDSS absolute $i$-band magnitude) vs. $\log R$ (the logarithm of radio loudness), compared to high-redshift, moderately radio-loud quasars and RQQs. The filled stars show our Chandra Cycle 12 targets, while the filled squares are HRLQs at $z>4$ with sensitive archival X-ray coverage. The asterisks show the high-redshift, moderately radio-loud quasars reported in Bassett et al. (2004), Lopez et al. (2006), and M11. The small dots represent the high-redshift, radio-quiet SDSS quasars that have sensitive X-ray coverage. All the symbols are color-coded based on their redshifts using the color bar at the top right corner of the figure. The dotted and dashed lines show our criteria for RLQs (log $R \geqslant 1)$ and highly radio-loud quasars $(\log R>2.5)$. The dash-dotted curve shows the quasar radio-loudness distribution from Ivezić et al. (2004), which illustrates that our sample represents the quasars residing in the tail of high radio loudness. 


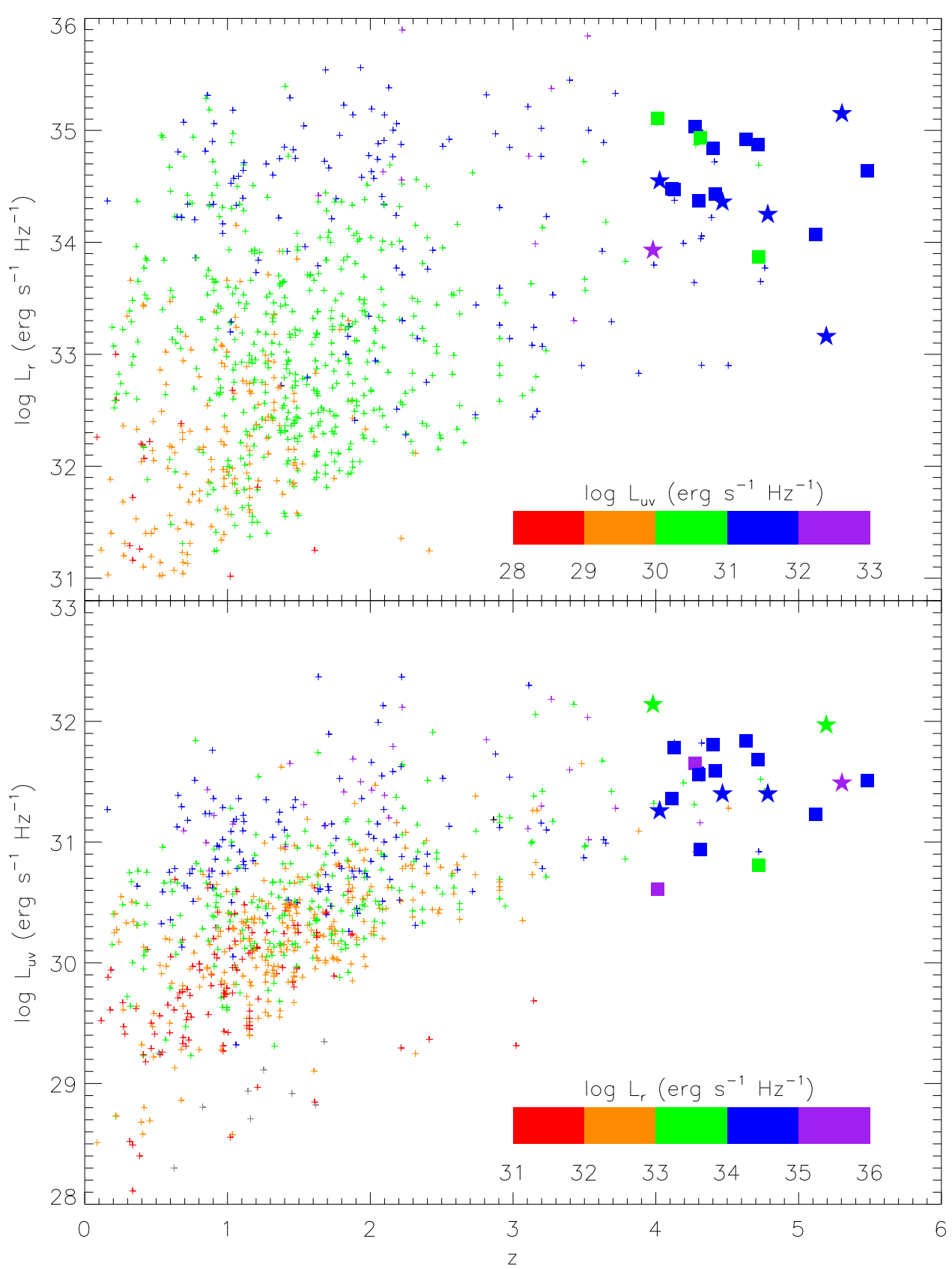

FIG. 2.- The monochromatic luminosity at rest-frame $5 \mathrm{GHz}$ (radio; upper panel) and at rest-frame $2500 \AA$ (UV; lower panel), plotted against redshift. The filled stars show our Chandra Cycle 12 targets, while the filled squares are our HRLQs with sensitive archival X-ray coverage. The plus signs represent the radio-loud and radio-intermediate objects in the full sample of M11. The upper (lower) panel is color-coded based on UV (radio) luminosity using the color bars at the bottom right corner of each panel. Our sample of quasars are among the most luminous objects in both the radio and UV bands. 


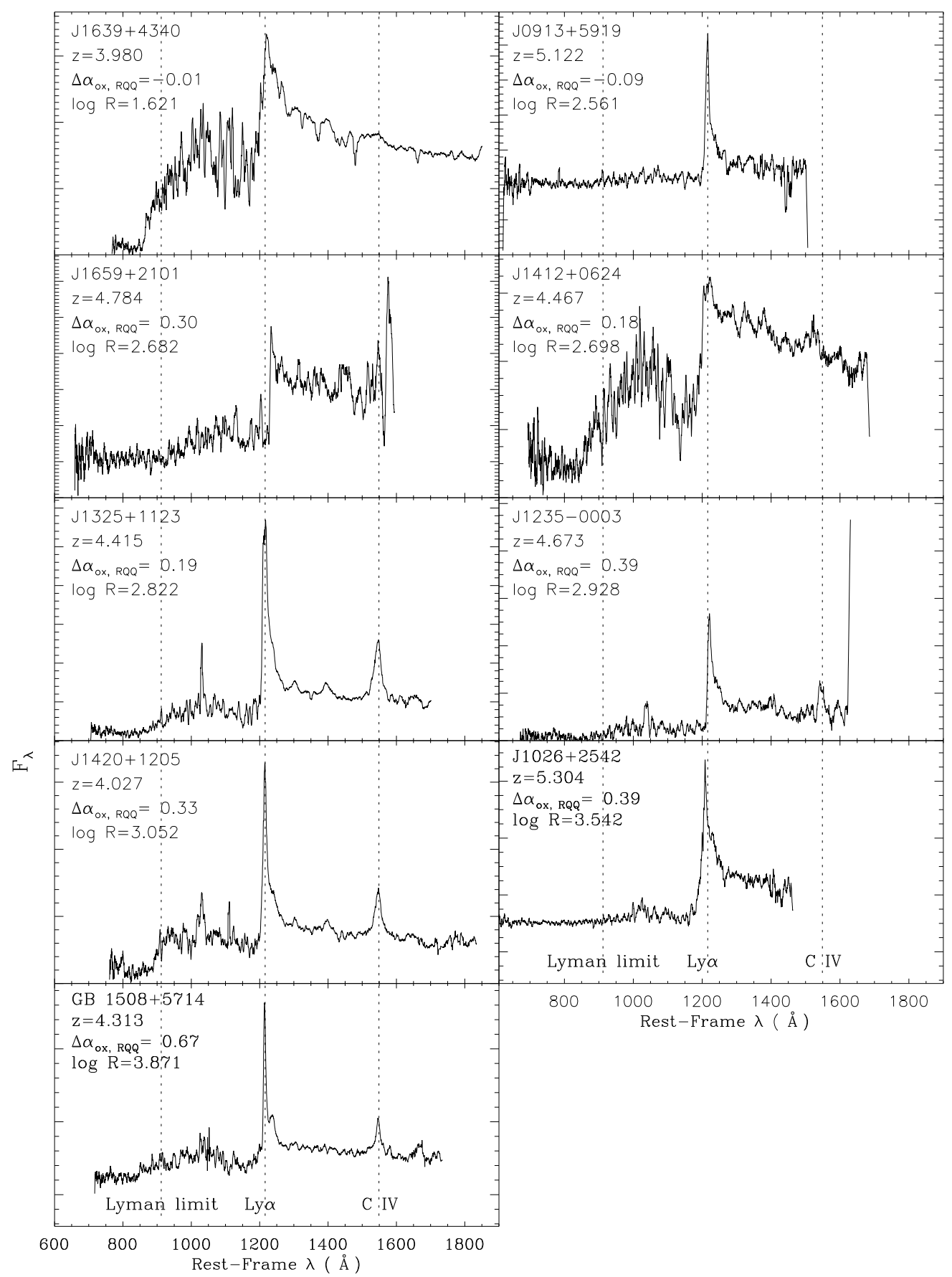

FIG. 3.- The SDSS spectra of five of our Chandra Cycle 12 targets and four objects with sensitive archival X-ray coverage, ordered by log $R$ which is shown at the top-left corner of each panel. Also labeled are the object names and redshifts. The $y$-coordinates are the flux density $\left(F_{\lambda}\right)$ in linear units. The spectra have been smoothed using a 20-pixel sliding-box filter. The wavelengths corresponding to major emission lines (Ly $\alpha \lambda 1216$ and C IV $\lambda 1549$ ) and the Lyman limit are labeled by the dotted vertical lines. The spectral resolution is $R \approx 2000$. The apparent broad features of J1639+4340 and J1412+0624 at $\sim 1000 \AA$ are caused by the stretched scaling due to their weak $\operatorname{Ly} \alpha$ emission lines. 
HIGHLY RADIO-LOUDQUUASARS AT $z>4$

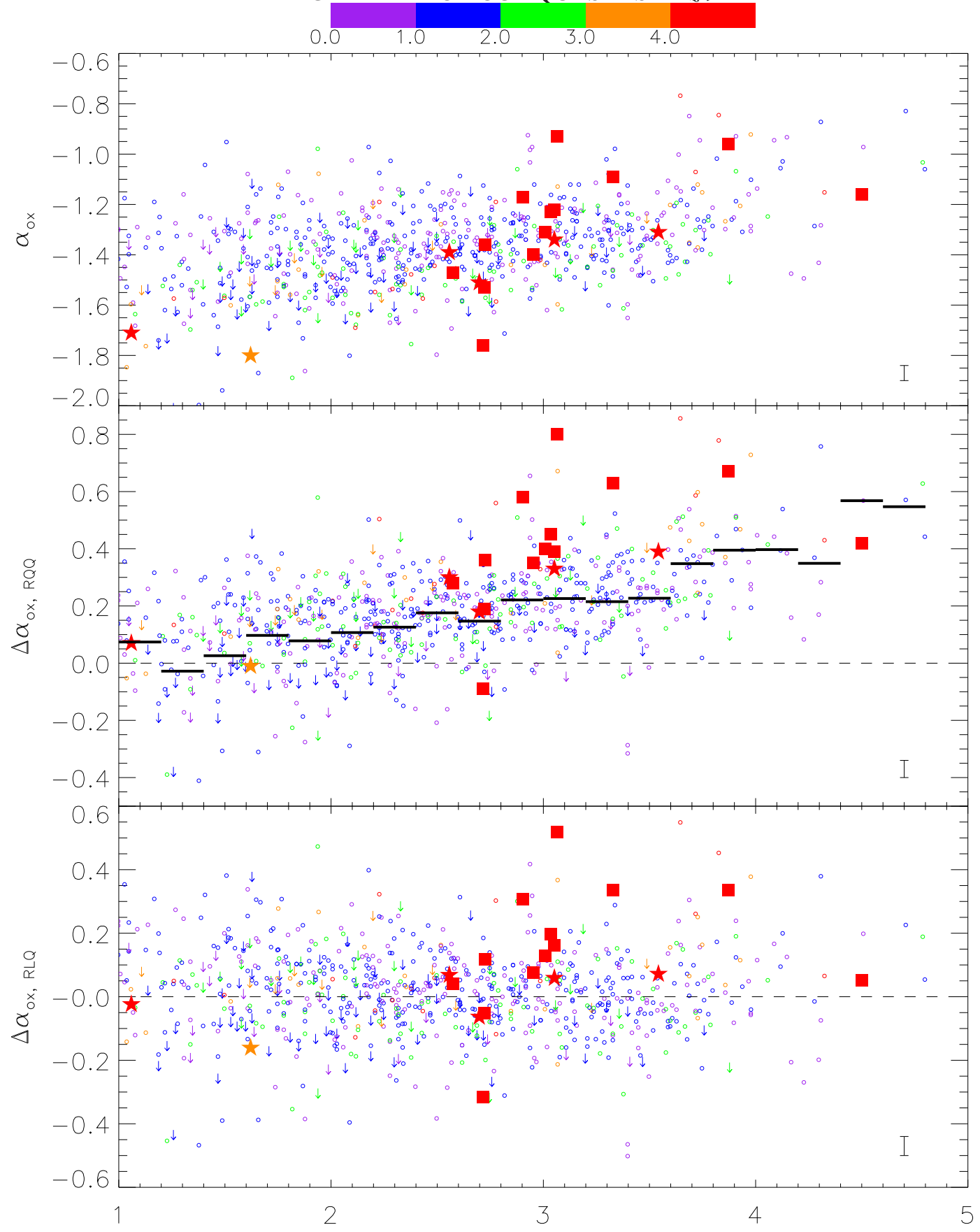

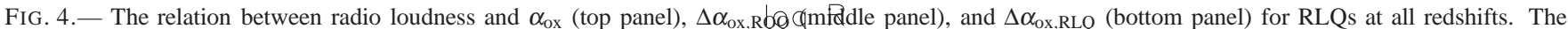
filled stars show our Chandra Cycle 12 targets, while the filled squares are our HRLQs with sensitive archival X-ray coverage. The open circles (downward arrows) represent the radio-loud and radio-intermediate objects in the full sample of M11 that have X-ray detections (upper limits). The typical error bars for $\alpha_{\mathrm{ox}}$,

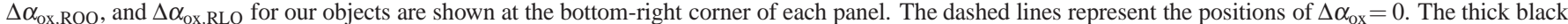
lines show the mean $\Delta \alpha_{\mathrm{OX}, \mathrm{RQQ}}$ values for the M11 objects binned in $\log R(\Delta \log R=0.2$ per bin). All symbols are color-coded based on their redshifts using the color bar at the top of the figure. 


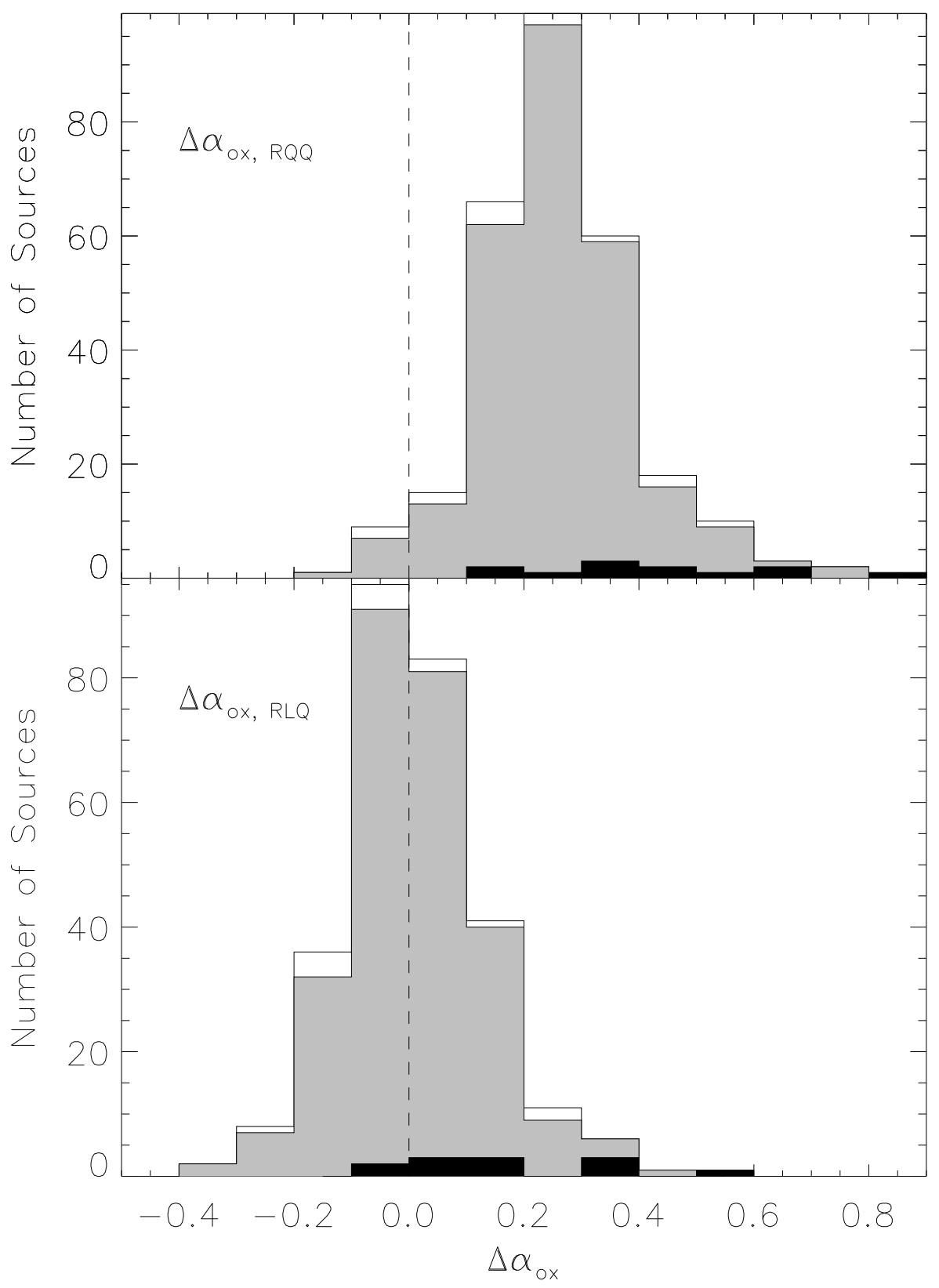

FIG. 5. - The histograms of $\Delta \alpha_{\mathrm{ox}, \mathrm{RQQ}}$ (top panel) and $\Delta \alpha_{\mathrm{ox}, \mathrm{RLQ}}$ (bottom panel) for the full-sample objects in M11 with $\log R>2.5, z<4$, and $m_{i}<20$ (grey and open histograms for X-ray detected and undetected objects, respectively) and our HRLQs at $z>4$ with $m_{i}<20$ (black histogram). 


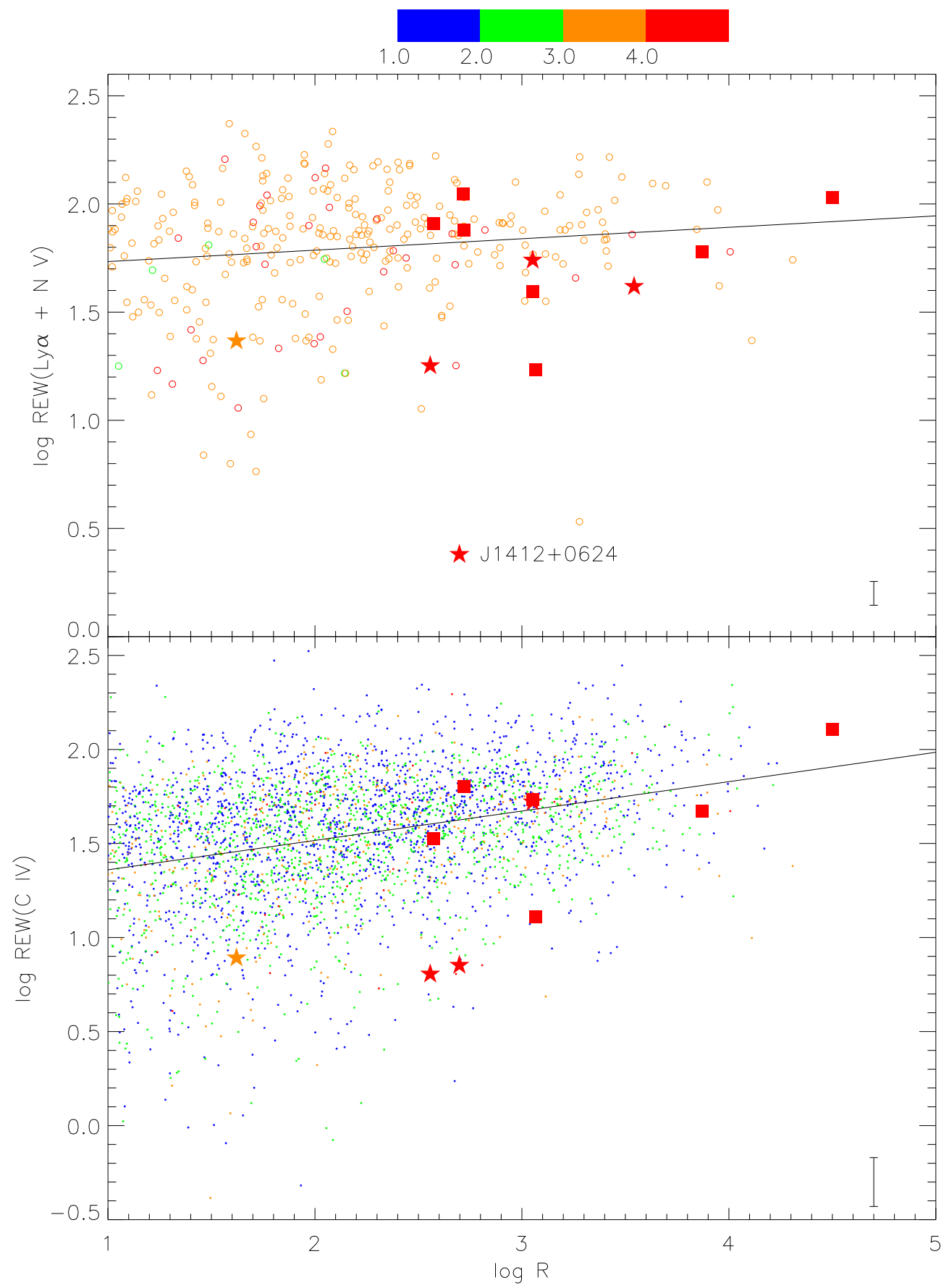

FIG. 6. - The REWs of the broad emission lines Ly $\alpha+\mathrm{N} v$ (upper panel) and C IV (lower panel) as a function of radio loudness. In the upper panel, the open circles represent the radio-loud SDSS DR5 quasars in Table 1 of Diamond-Stanic et al. (2009) which gives the REW measurements of Ly $\alpha+\mathrm{N}$ V. In the lower panel, the small dots represent the radio-loud SDSS DR7 quasars with C IV REW measurements from Shen et al. (2011). Our objects with available line REW measurements are shown in both panels as filled stars (for Chandra Cycle 12 targets) or filled squares (archival objects). The weak-line quasar in our HRLQ sample (J1412+0624) is labeled in the upper panel. The typical error bars for emission-line REWs of our objects are shown at the bottom-right corner of each panel. All symbols are color-coded based on their redshifts using the color bar at the top of the figure. 


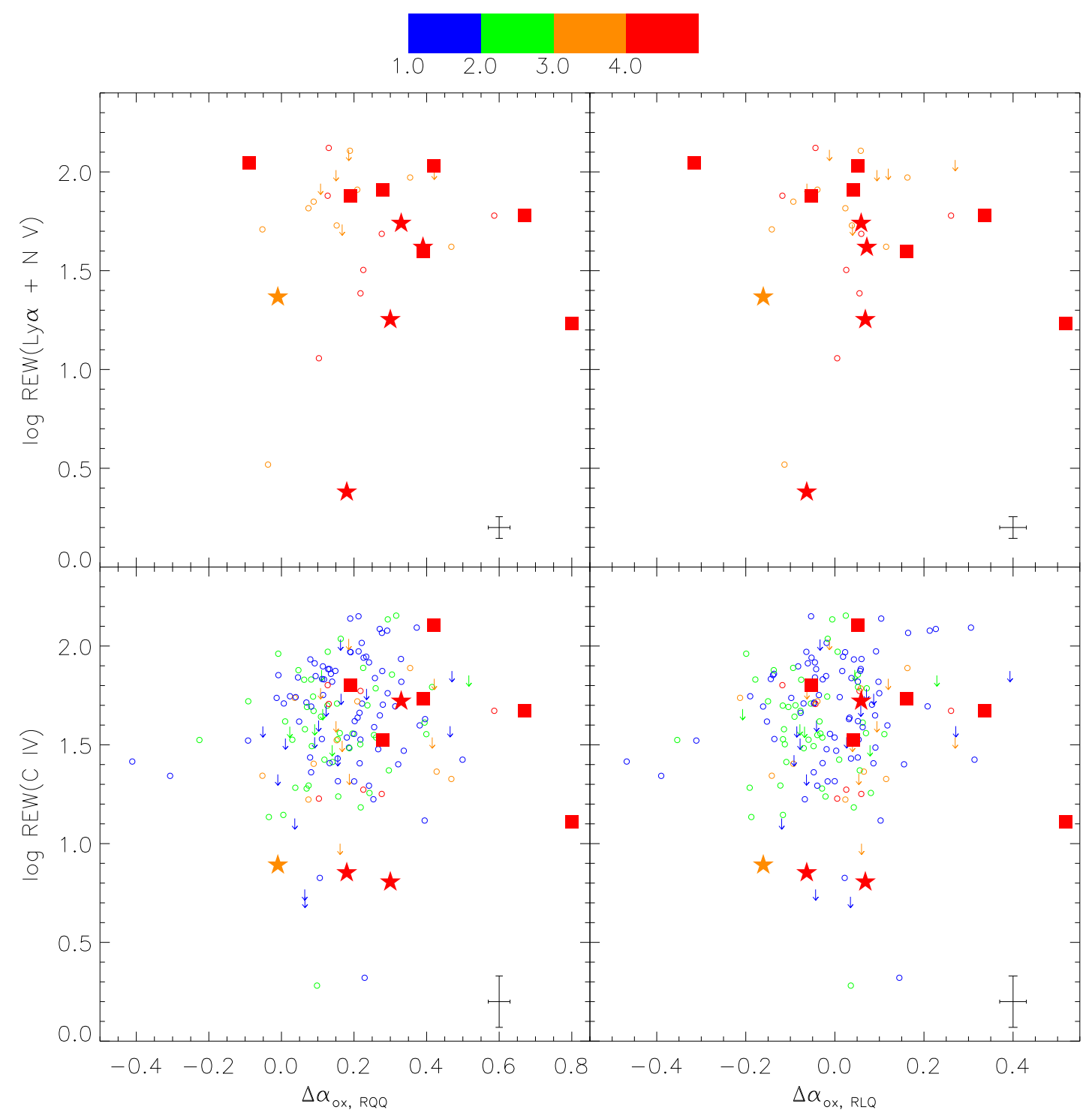

FIG. 7.- The REWs of the broad emission lines Ly $\alpha+\mathrm{N}$ v (top row) and C IV (bottom row) as a function of $\Delta \alpha_{\text {ox,RQQ }}$ (left column) and $\Delta \alpha_{\text {ox,RLQ }}$ (right column), respectively. The open circles in the top-row panels represent the M11 objects with available REW(Ly $\alpha+\mathrm{N}$ V) measurements in Table 1 of DiamondStanic et al. (2009). In the bottom-row panels, the open circles are the M11 objects with REW(C IV) measurements from Shen et al. (2011). Our objects with available line REW measurements are shown in both panels as filled stars (for Chandra Cycle 12 targets) or filled squares (archival objects). The typical error bars for emission-line REWs, $\Delta \alpha_{\mathrm{ox}, \mathrm{RQQ}}$, and $\Delta \alpha_{\mathrm{ox}, \mathrm{RLQ}}$ of our objects are shown at the bottom-right corner of each panel. All symbols are color-coded based on their redshifts using the color bar at the top of the figure. 


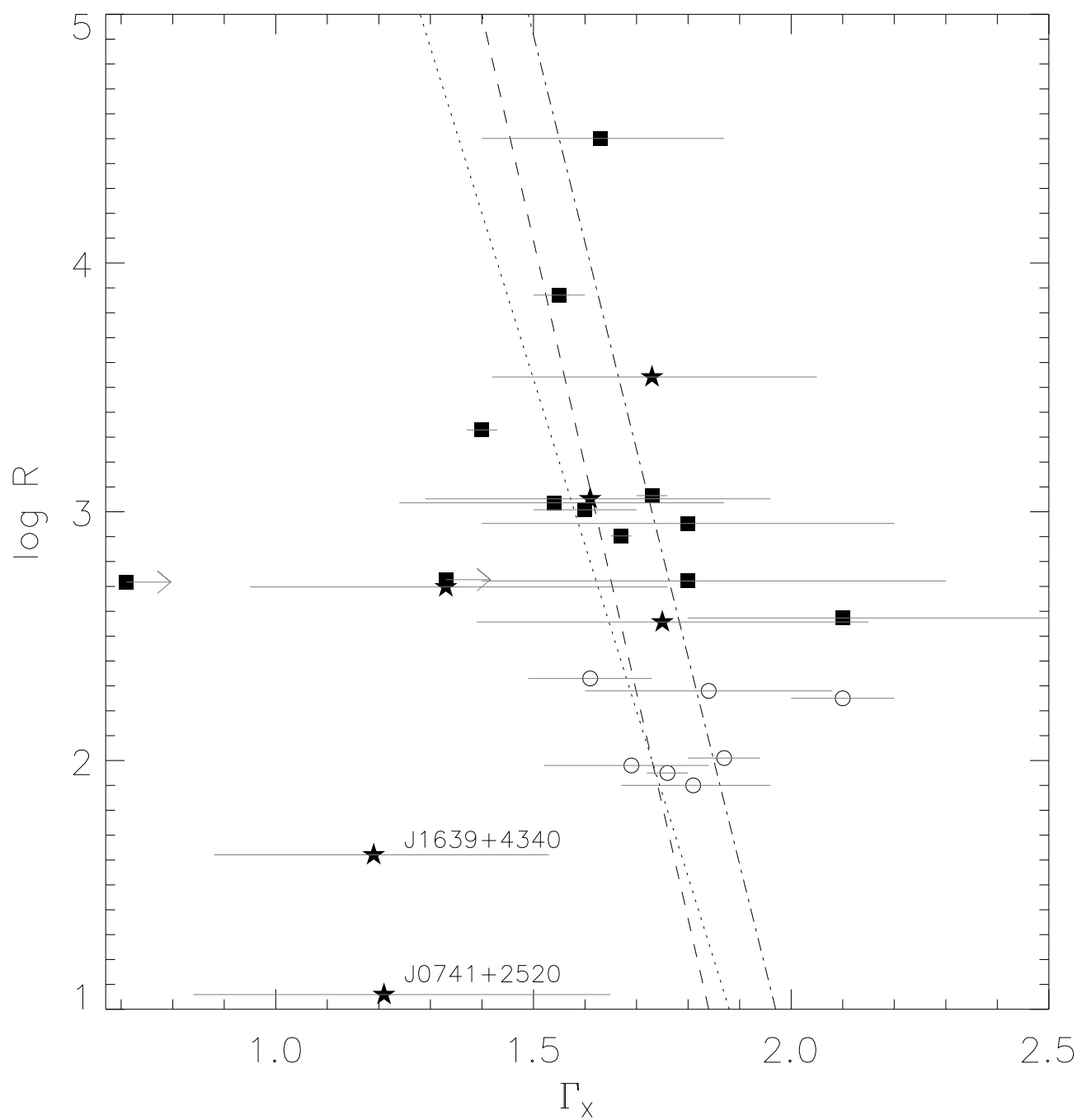

FIG. 8.- The relation between X-ray power-law photon index $\Gamma_{\mathrm{X}}$ and radio loudness $\log R$. The filled stars show our Chandra Cycle 12 targets, while the filled squares show our HRLQs with sensitive archival X-ray coverage. The open circles represent $z>4$ moderately radio-loud quasars in Saez et al. (2011). The grey horizontal lines (rightward arrow) represent the $1 \sigma$ error bars (lower limit) for the X-ray power-law photon indices. The dashed line shows our best-fit correlation between $\Gamma_{\mathrm{X}}$ and $\log R$ for $z \gtrsim 4 \mathrm{RLQs}$, while the dotted line and dash-dotted line represent the $\Gamma_{\mathrm{X}}-\log R$ correlations for $z>2$ RLQs in Saez et al. (2011) and for $z<2$ RLQs in Reeves \& Turner (2000), respectively. The two moderately radio-loud objects among our Chandra Cycle 12 targets are apparent outliers (J0741+2520 and J1639+4340; see the two filled stars labeled in the bottom left part of the figure). 


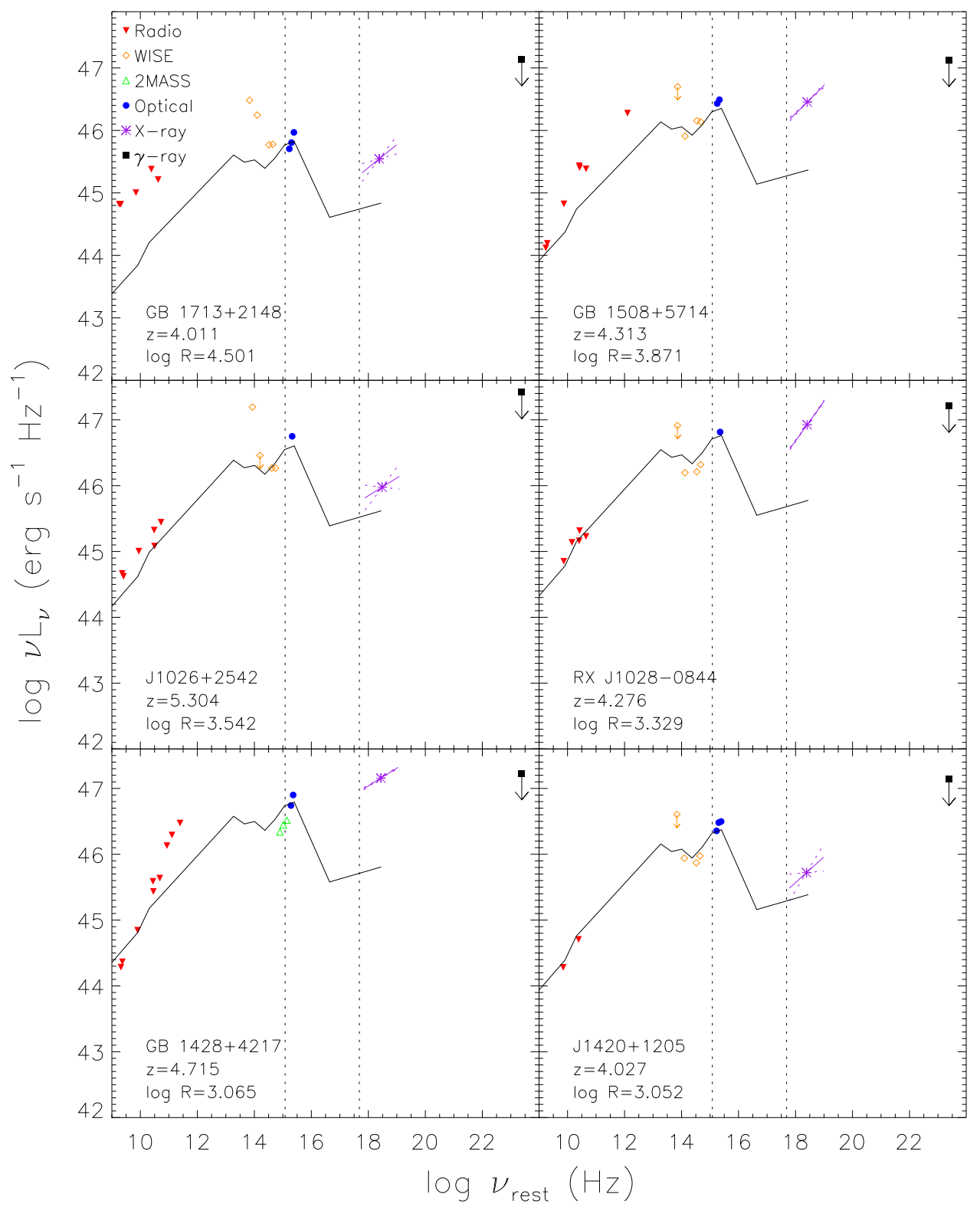

FIG. 9.- The rest-frame broad-band SEDs for our sample of objects ordered by $\log R$ (in descending order), including radio (red filled upside-down triangles), mid-infrared (WISE, orange open diamonds), near-infrared (2MASS and UKIRT, green open triangles), optical (blue filled circles), X-ray (purple asterisks and lines), and $\gamma$-ray (filled square) data points. Downward arrows indicate upper limits. The purple solid (dotted) lines show the X-ray power-law spectra (and their uncertainty range) based on the photon-index values provided in Column 9 of Table 4 The purple asterisks represent observed-frame $2 \mathrm{keV}$. The black solid lines show the composite SEDs for the 10 RLQs in Shang et al. (2011; S11) with comparable optical luminosity and radio loudness, scaled to the flux density at rest-frame $2500 \AA$ (corresponding to $10^{15.1} \mathrm{~Hz}$; see 4.4 . The vertical dotted lines show the frequencies of rest-frame $2500 \AA$ and $2 \mathrm{keV}$. The name, redshift, and $\log R$ of each object are labeled at the bottom right of each panel. 


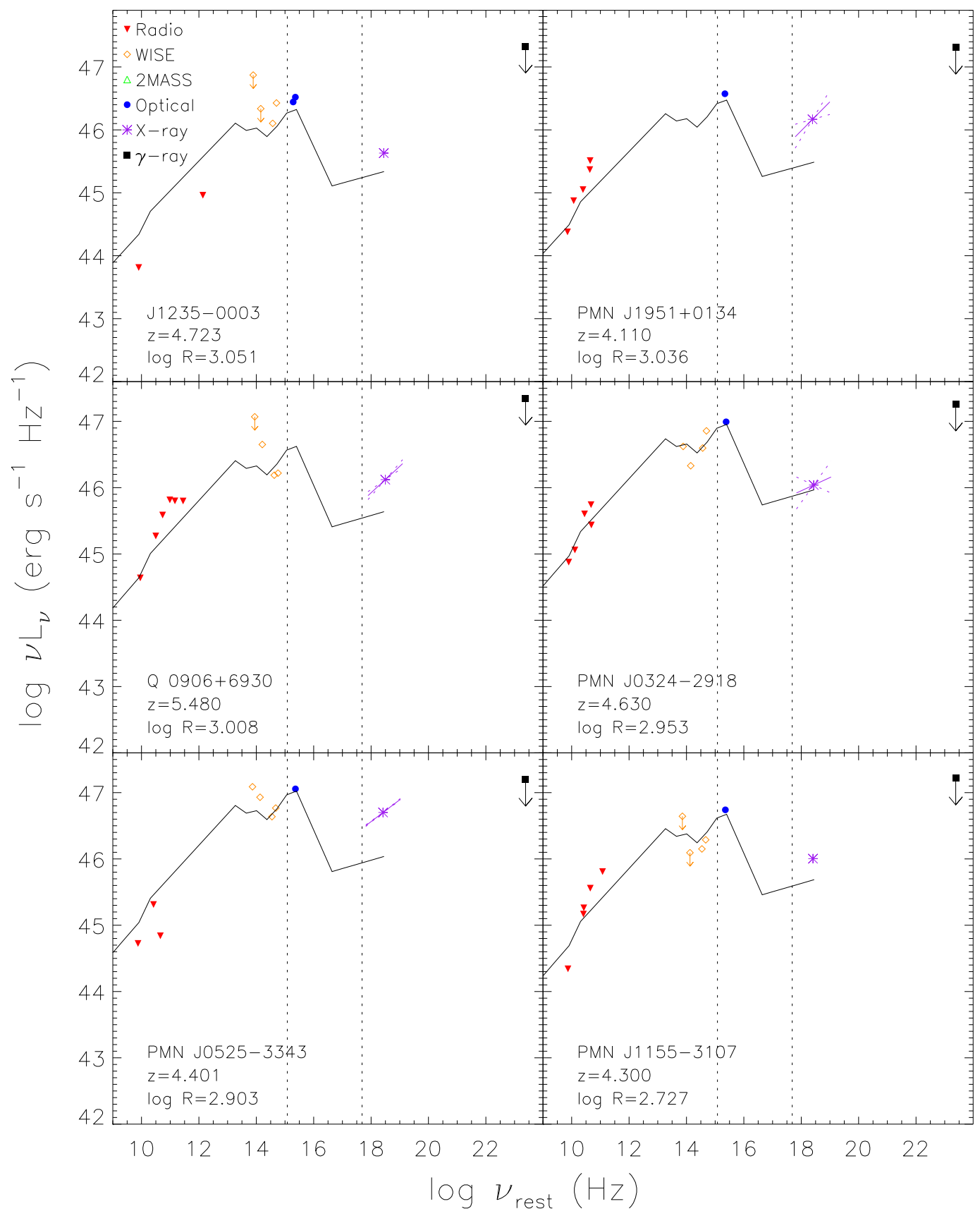

FIG.9-Continued. 


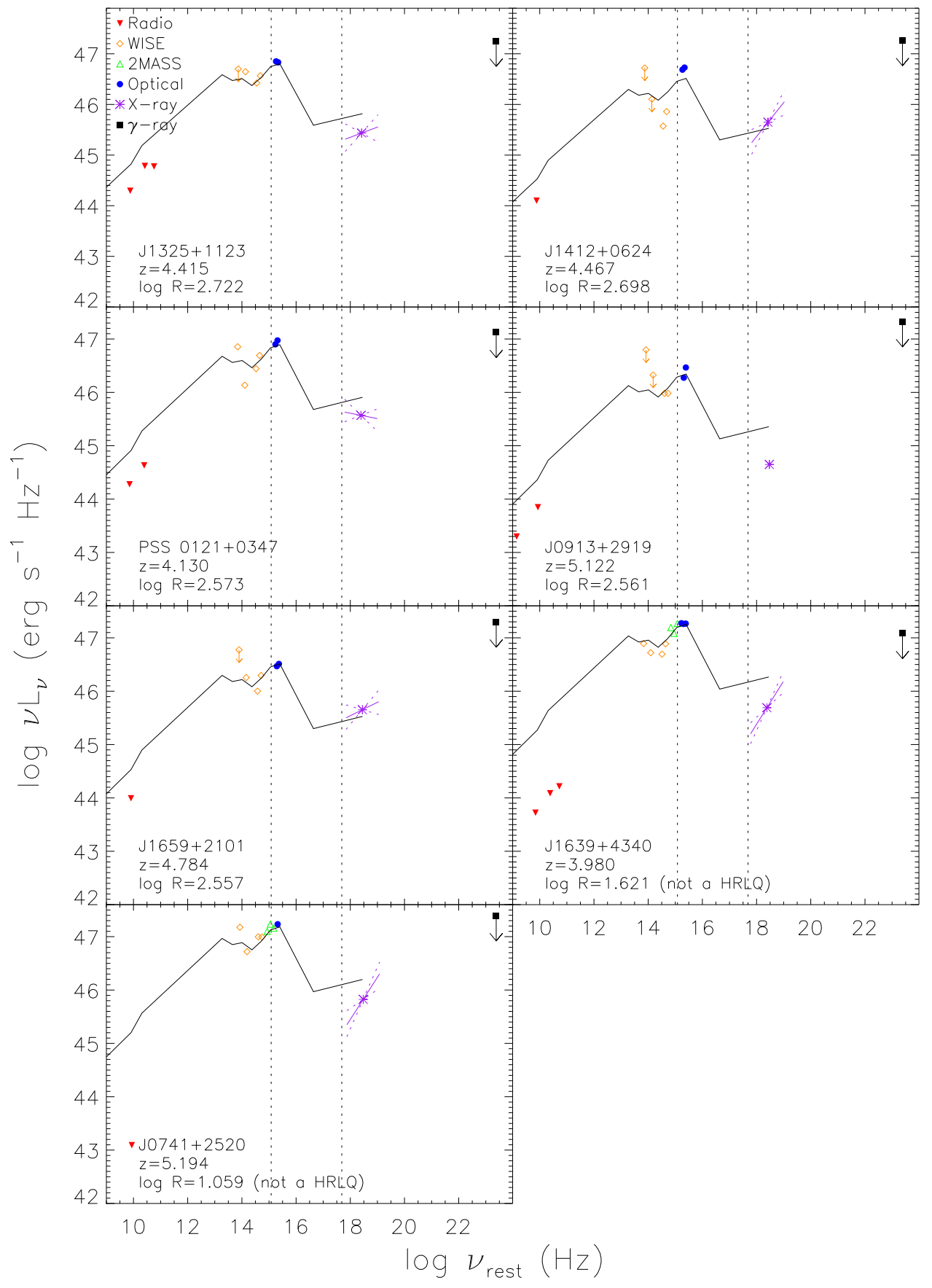

FIg.9-Continued. 